\title{
Green approach for visible light-induced direct functionalization of 2-methylquinolines
}

Juliana A. Dantas, Radell Echemendía, Marilia S. dos Santos, Márcio W. Paixão, Marco A. B. Ferreira* and Arlene G. Corrêa*

Centre of Excellence for Research in Sustainable Chemistry, Department of Chemistry, Federal University of São Carlos, São Carlos, SP, Brazil, 13565-905. *e-mail: agcorrea@ufscar.br and marcoantbf@gmail.com 


\section{TABLE OF CONTENTS}

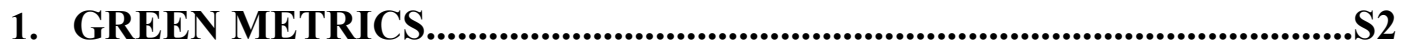

2. PHOTOCHEMICAL REACTOR....................................................................

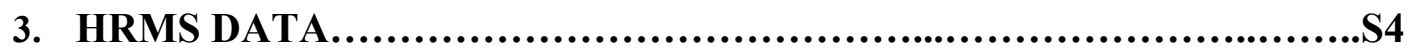

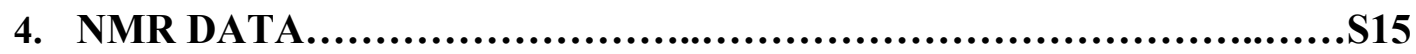

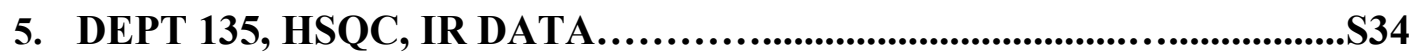

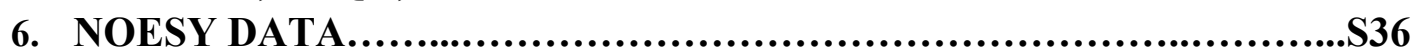

7. DEUTERIUM-LABELING EXPERIMENTS..............................S38

8. TRAPPING EXPERIMENT: MS ANALYSIS.............................S39

9. THEORICAL MECHANISTIC STUDIES................................S41

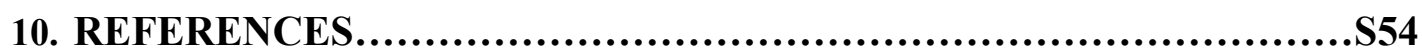




\section{GREEN CHEMISTRY METRIC}

The efficiency of our method was calculated by mathematical relationships between step and cumulative process mass intensities (PMIs) using the method described by Andraos. ${ }^{1}$ This metric covers all mass based inputs, as solvents, catalysts, reagents, work up and others. Some parameters could be correlated as atom economy $(\mathrm{AE})^{2}$ and reaction mass efficiency $(\mathrm{RME})^{3}$. To calculate the metrics, the following procedure was used:

In a $20 \mathrm{~mL}$ sealed vial were added the heterocycle $1 \mathrm{a}(0.5 \mathrm{mmol}, 1$ eq. $), \mathrm{LiClO}_{4}(1$ mmol, 2 eq.) and dry EtOAc $(1.0 \mathrm{~mL})$ under magnetic stirring until complete solubilization. Diacetyl 2a was added (7 eq., $0.3 \mathrm{~mL}$ ) and the reaction was maintained under magnetic stirring and irradiation with blue LED $(37 \mathrm{~W})$ for $48 \mathrm{~h}$ with monitored temperature around $30-35^{\circ} \mathrm{C}$. Consumption of the starting materials was observed by TLC. The crude mixture was transferred to a round flask using EtOAc $(5.0 \mathrm{~mL})$ and then concentrated under vacuum. The desired product 3aa was purified by flash chromatography with silica gel (27 g) using Hexane/EtOAc (9:1) (200 mL) as eluent.

Table S1: Summary of green metrics for synthesis of 3aa.

\begin{tabular}{|l|r|}
\hline Metric & value \\
\hline Yield & 80.2 \\
\hline Conversion & 100.0 \\
\hline Selectivity & 80.2 \\
\hline AE & 68.3 \\
\hline RME & 19.2 \\
\hline PMI total & 1835.8 \\
\hline PMI Reaction & 10.1 \\
\hline PMI reactants, reagents, catalyst & 5.2 \\
\hline PMI reaction solvents & 4.9 \\
\hline PMI Workup & 1825.8 \\
\hline PMI Workup chemical & 293.5 \\
\hline PMI workup solvents & 1532.3 \\
\hline
\end{tabular}




\section{PHOTOCHEMICAL REACTOR}

These studies were carried out inside a cardboard box using a blue LED Kessil ${ }^{\mathrm{TM}}$ H150BLUE in a range of emission of 400 to $525 \mathrm{~nm}$ with two maximums at $421 \mathrm{~nm}$ and the higher at $452 \mathrm{~nm}$ and power at $37 \mathrm{~W}$ (Figure S1).

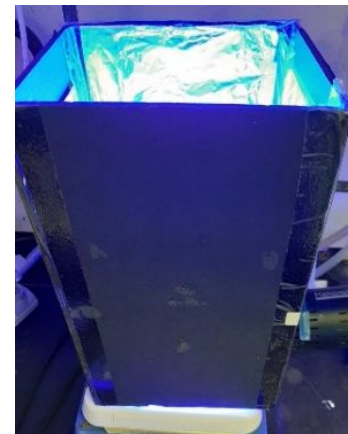

a)

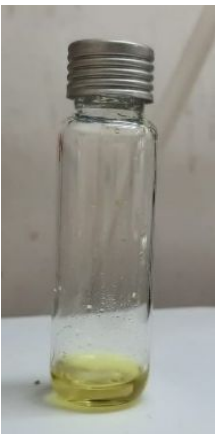

b)

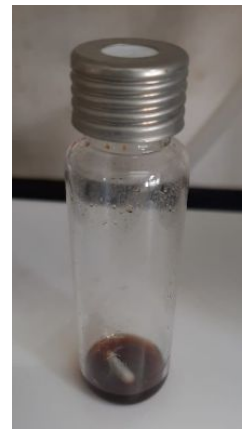

c)

Figure S 1: a) Photochemical reactor; b) Start of the reaction; c) End of the reaction.

The reaction vessel (headspace vial Supelco ${ }^{\mathrm{TM}}$ Analytical) was positioned at $10 \mathrm{~cm}$ far of the lamp without fan (Figure S2), so we did the measure of the temperature inside the reactor that was $30-35^{\circ} \mathrm{C}$.

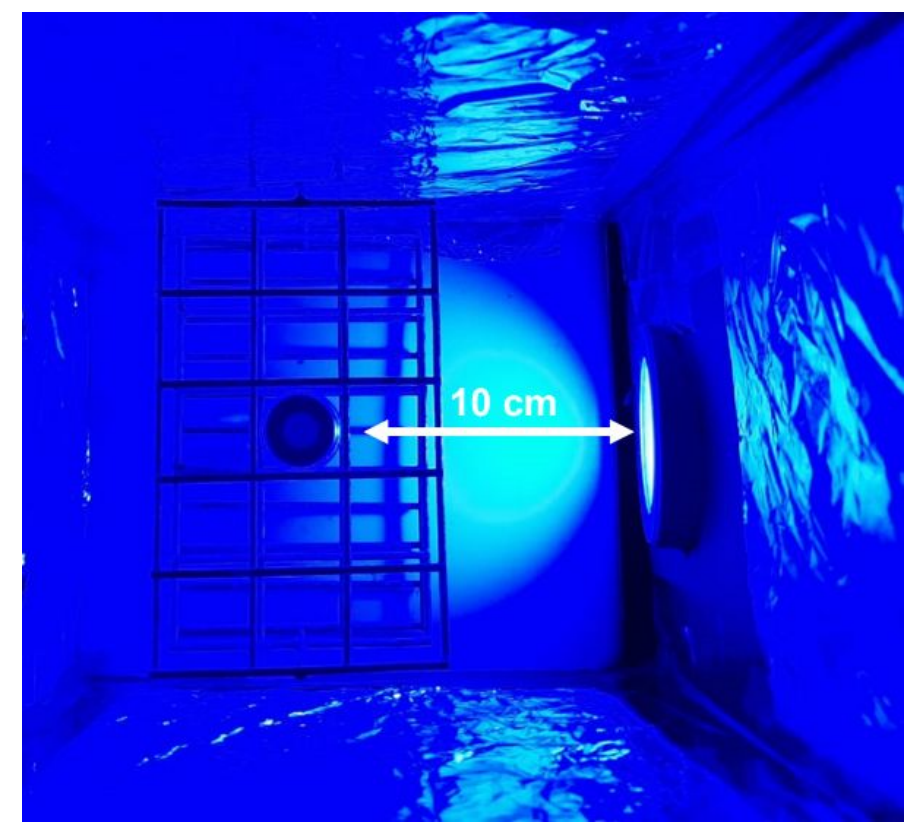

Figure S 2: Inside of the reactor. 
3. HRMS DATA

HRMS - ES+ $m / z:[\mathrm{M}+\mathrm{H}]^{+}$or $[\mathrm{M}+\mathrm{Na}]^{+}$or $[\mathrm{M}+\mathrm{K}]^{+}$

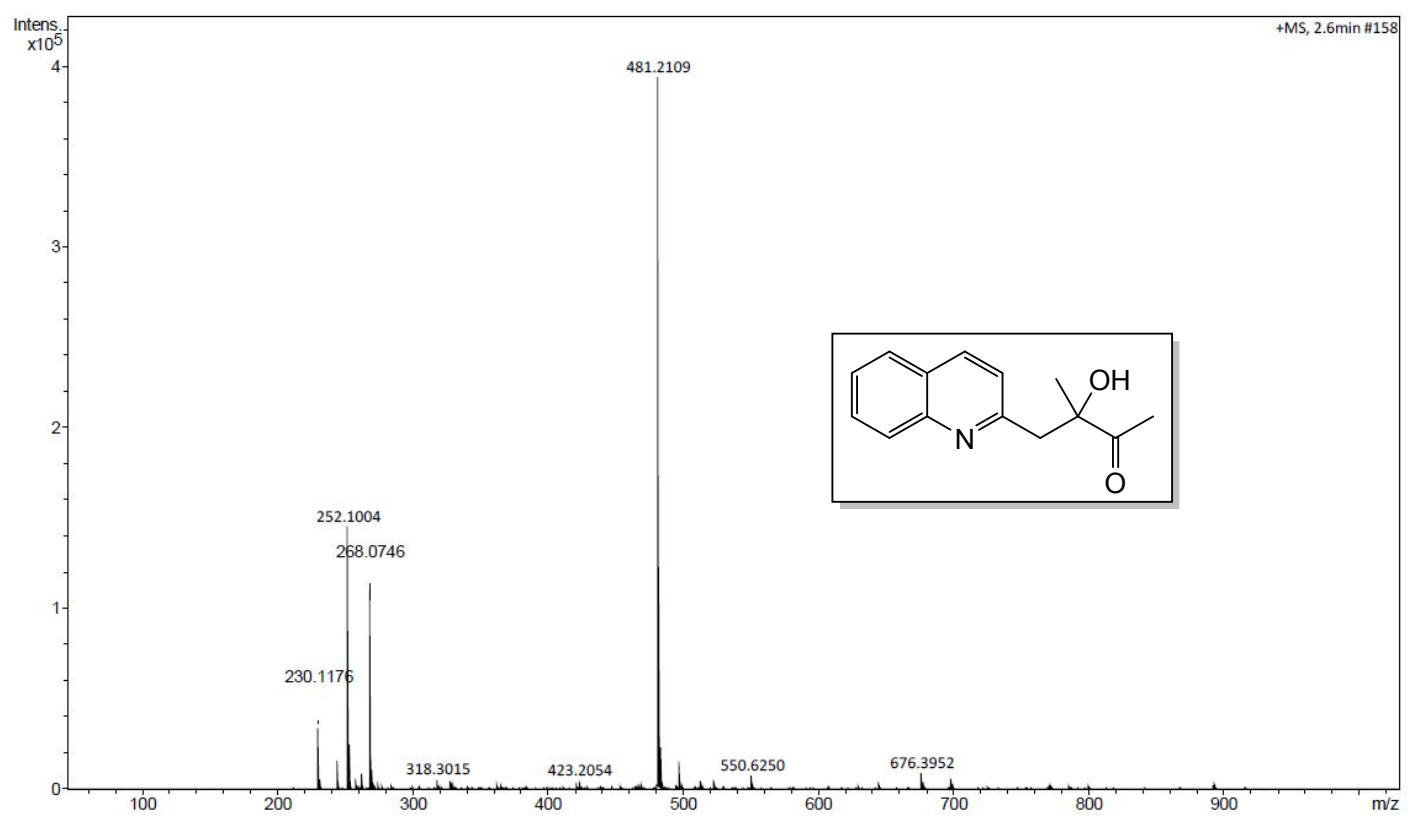

Figure S 3: 3-hydroxy-3-methyl-4-(quinolin-2-yl)butan-2-one (3aa)

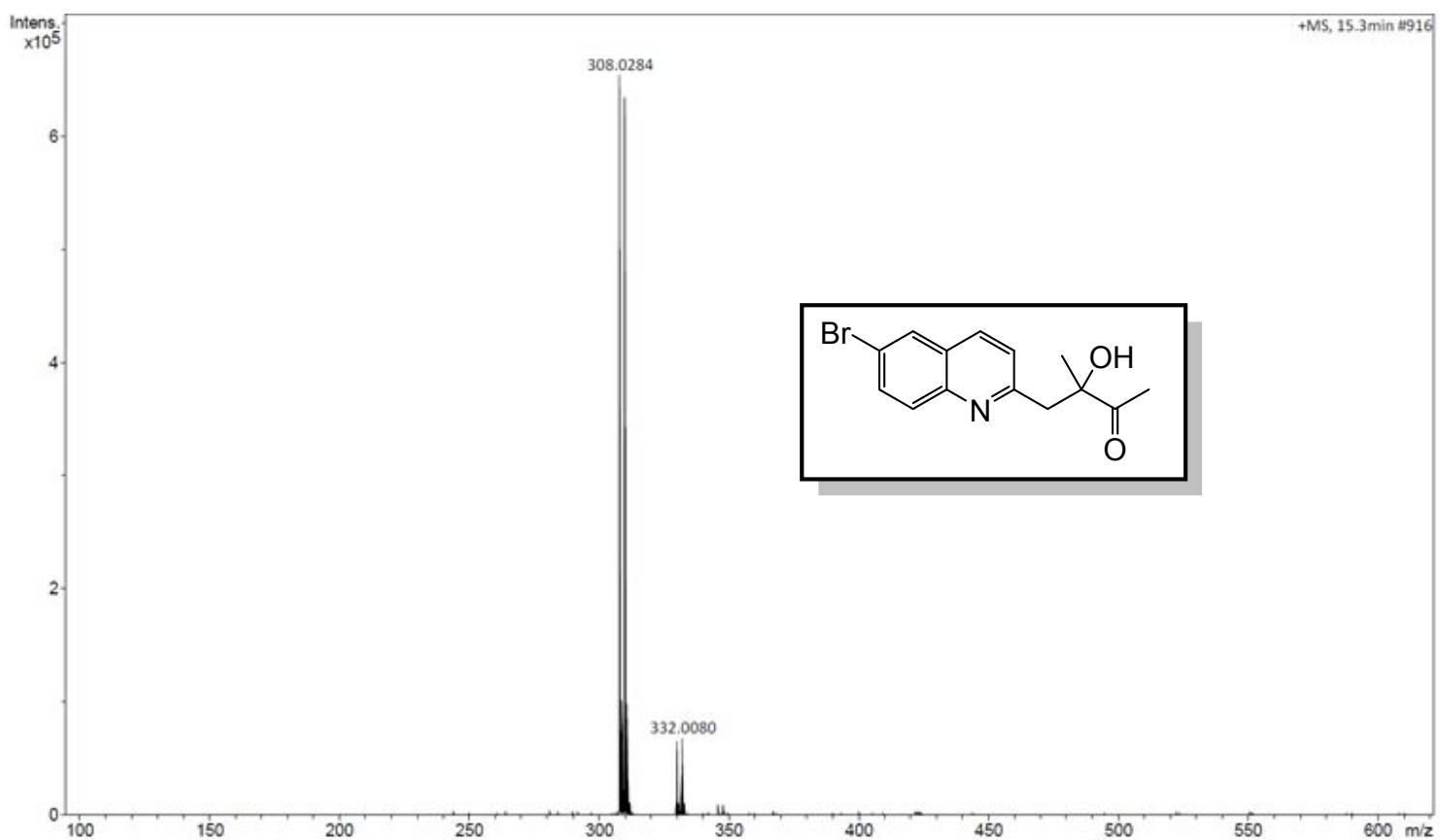


Figure S 4: 4-(6-bromoquinolin-2-yl)-3-hydroxy-3-methylbutan-2-one (3ba)

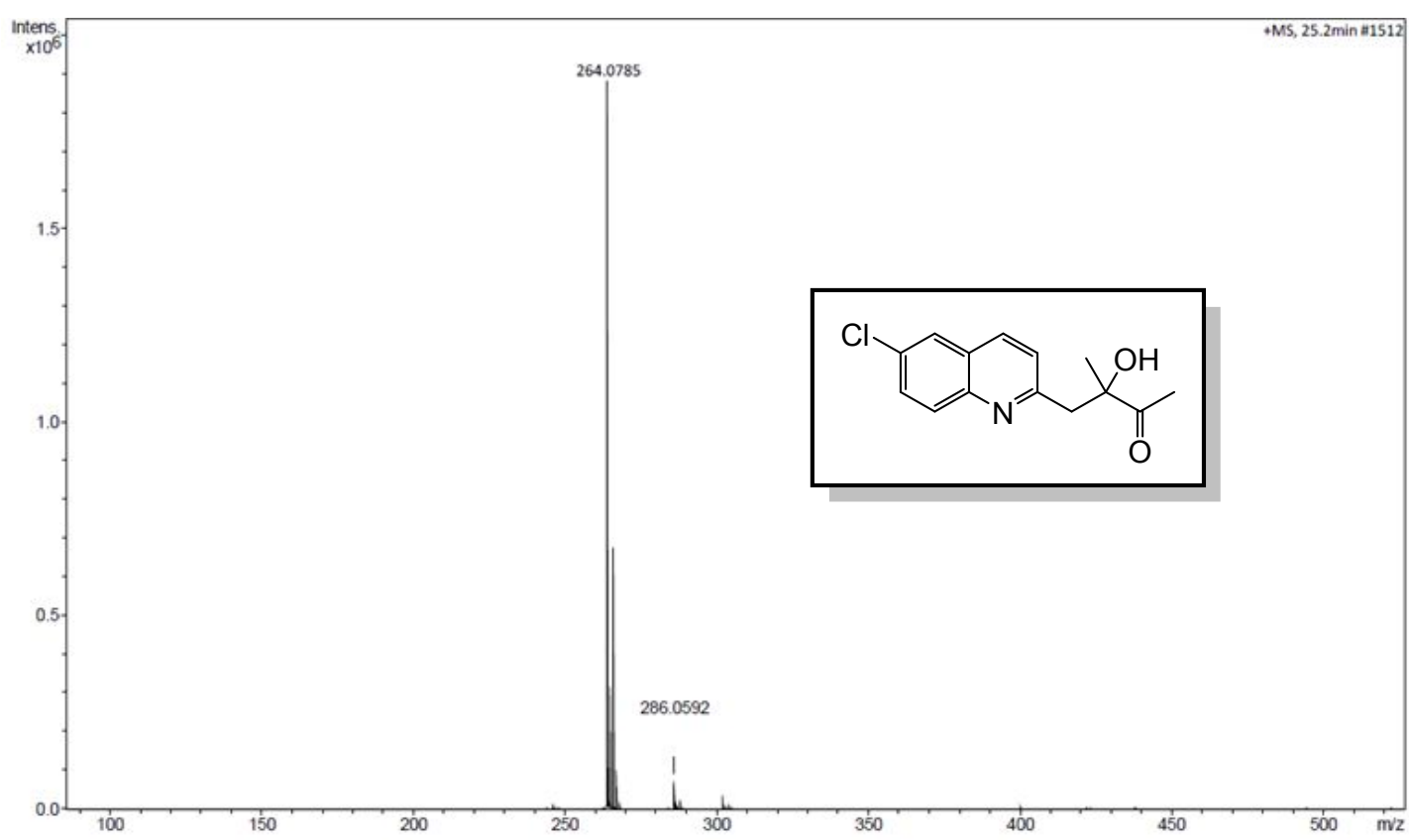

Figure S 5: 4-(6-chloroquinolin-2-yl)-3-hydroxy-3-methylbutan-2-one (3ca)

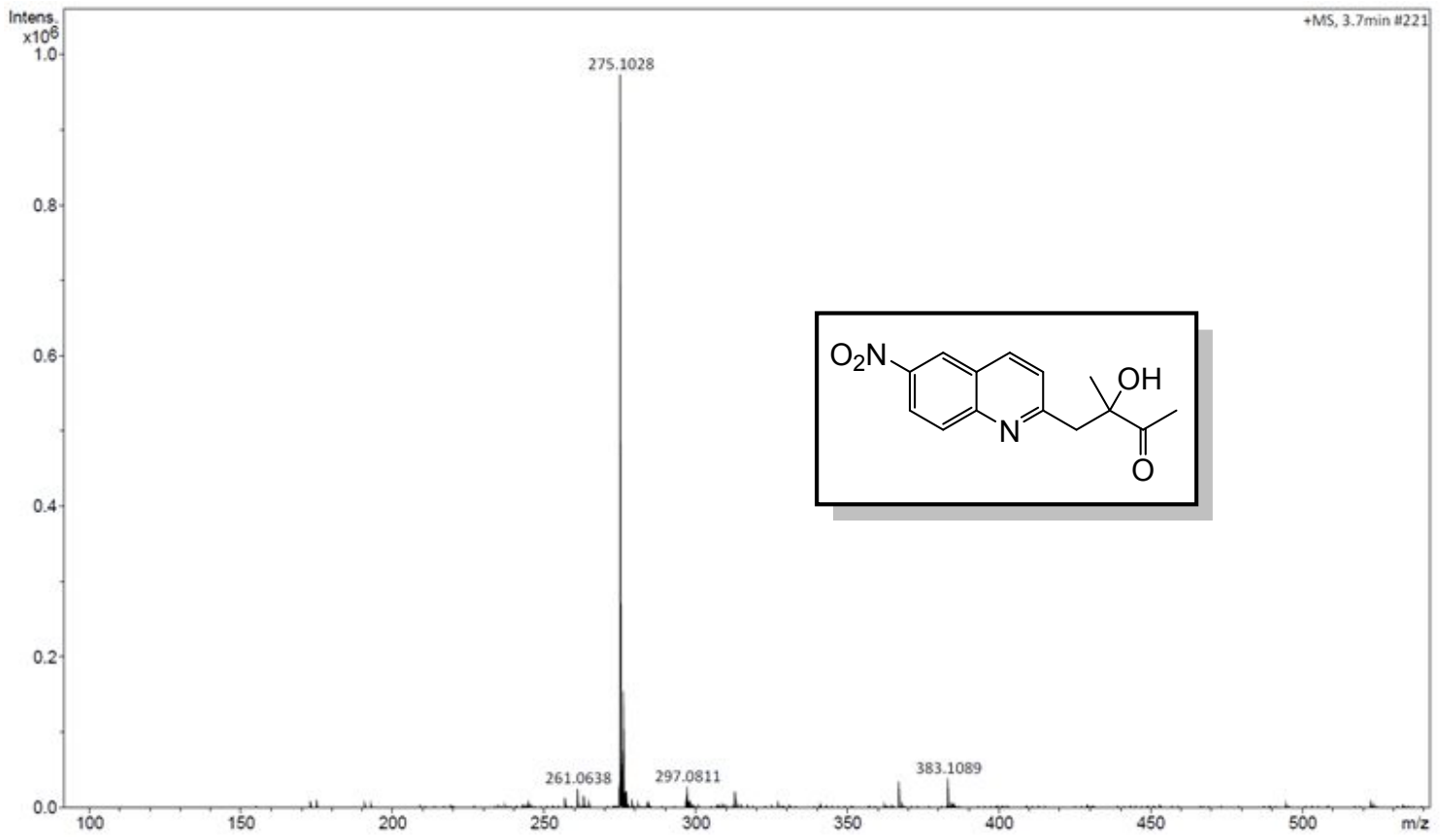

Figure S 6: 3-hydroxy-3-methyl-4-(6-nitroquinolin-2-yl)butan-2-one (3da) 


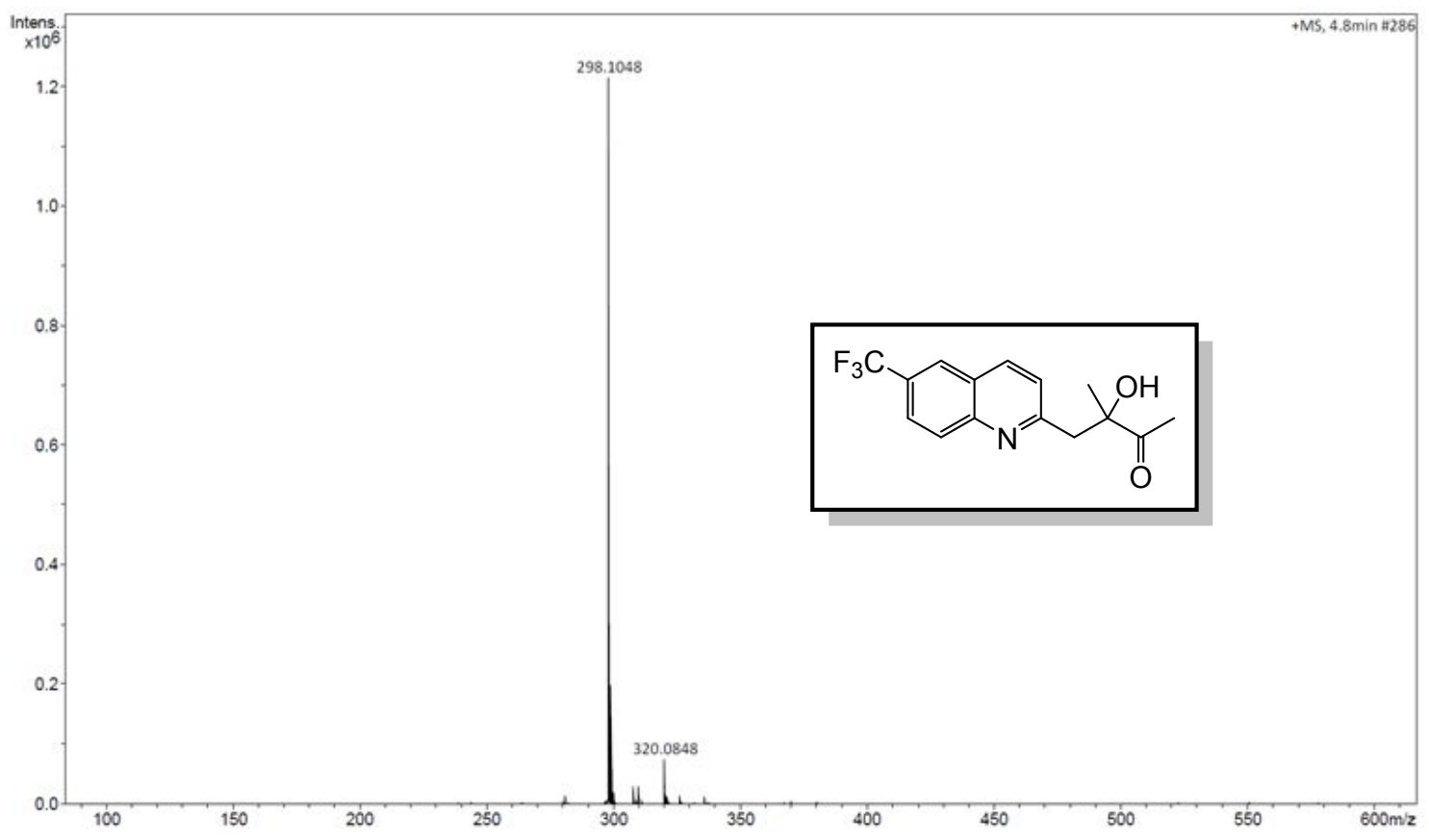

Figure S 7: 3-hydroxy-3-methyl-4-(6-(trifluoromethyl)quinolin-2-yl)butan-2-one (3ea)

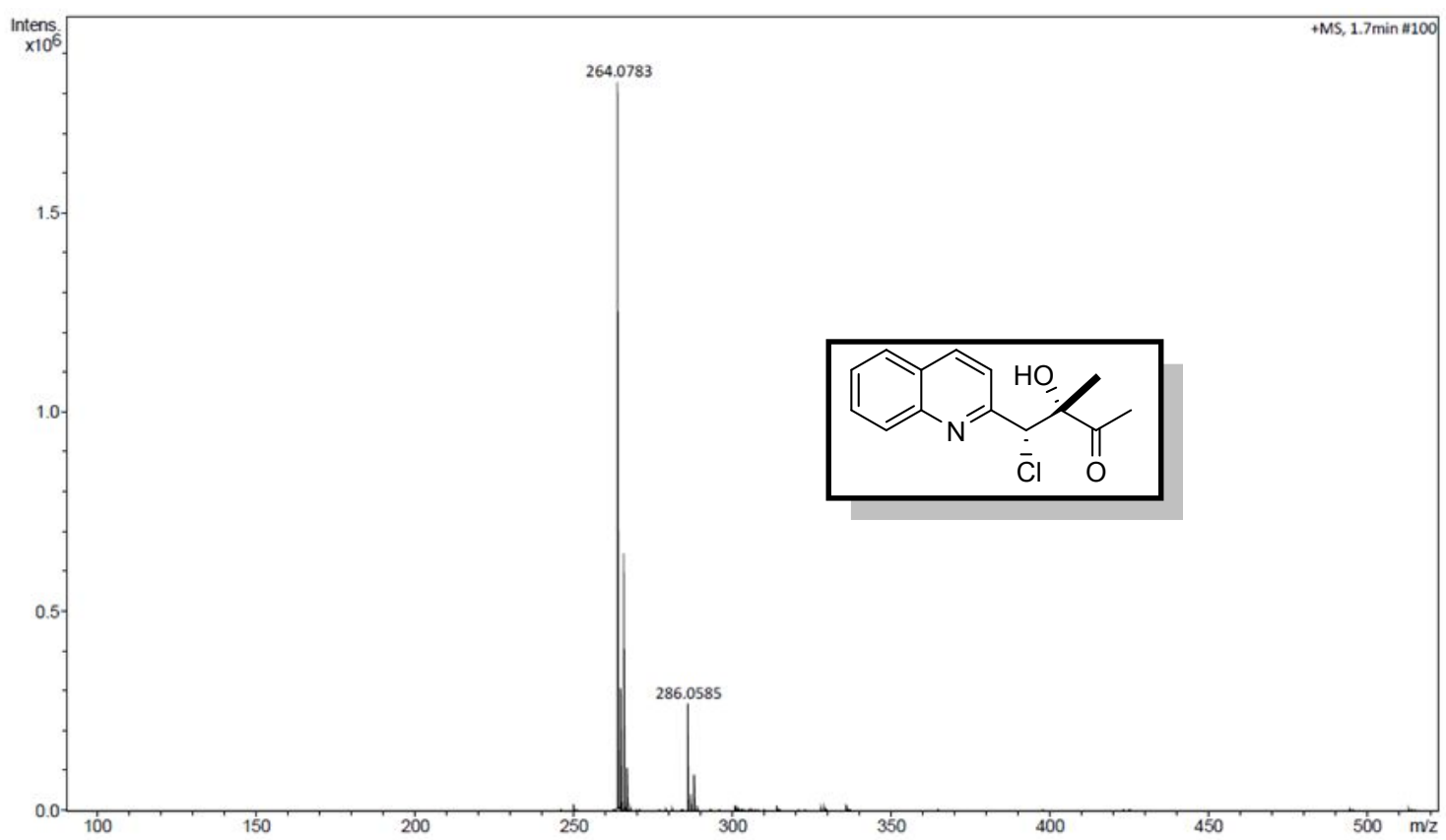

Figure S 8: 4-chloro-3-hydroxy-3-methyl-4-(quinolin-2-yl)butan-2-one (3fa) 


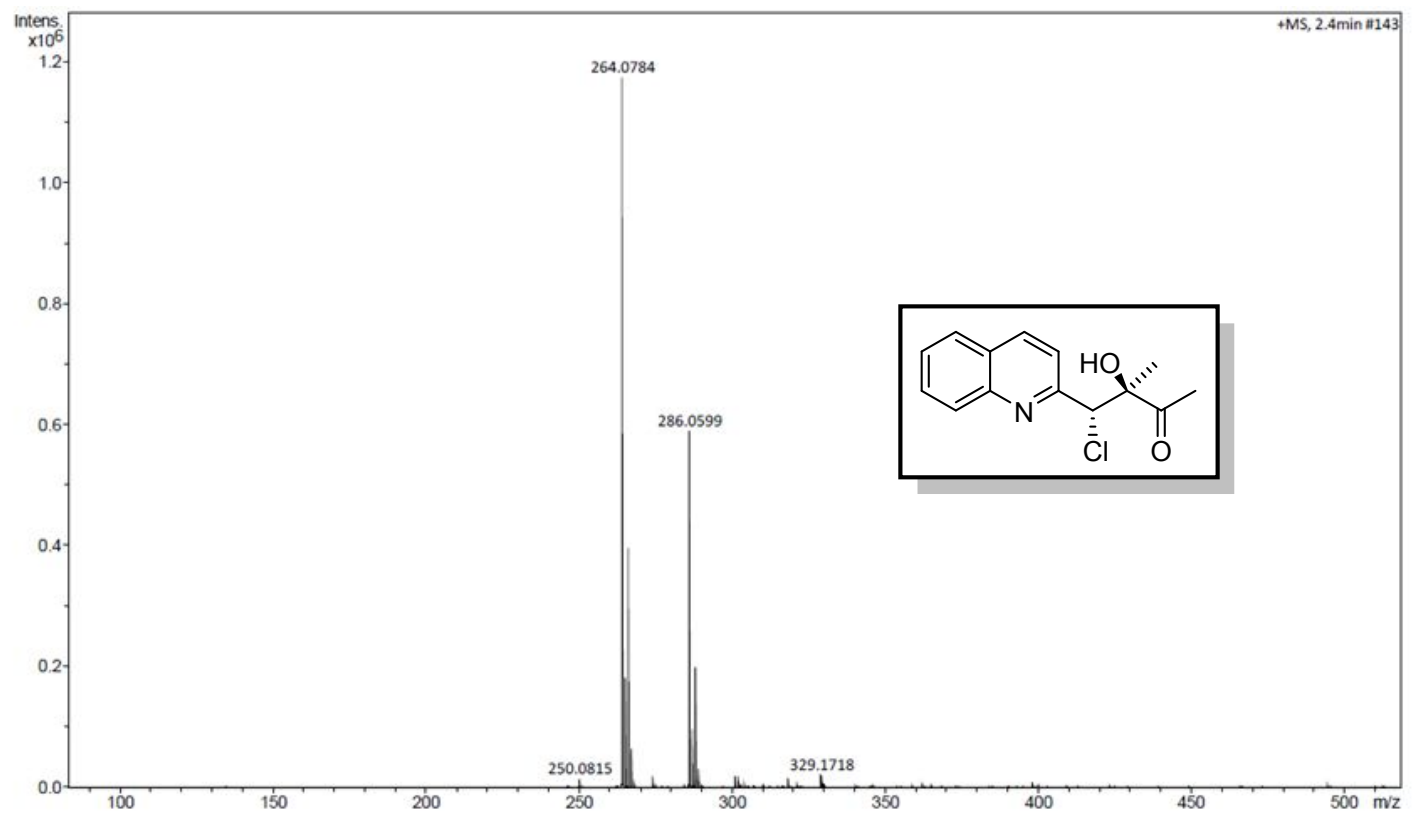

Figure S 9: 4-chloro-3-hydroxy-3-methyl-4-(quinolin-2-yl)butan-2-one (3fa')

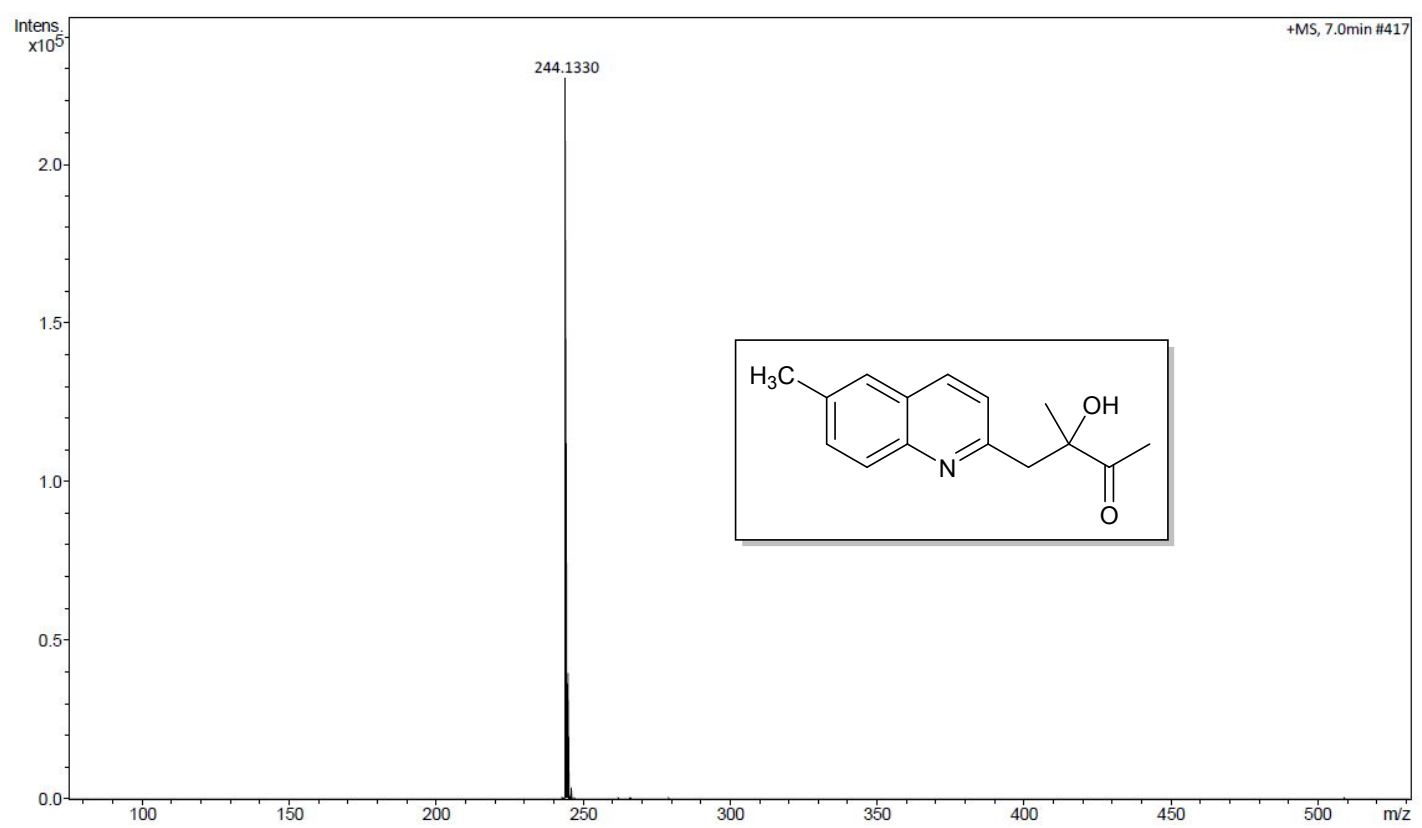

Figure S 10: 3-hydroxy-3-methyl-4-(6-methylquinolin-2-yl)butan-2-one (3ga) 


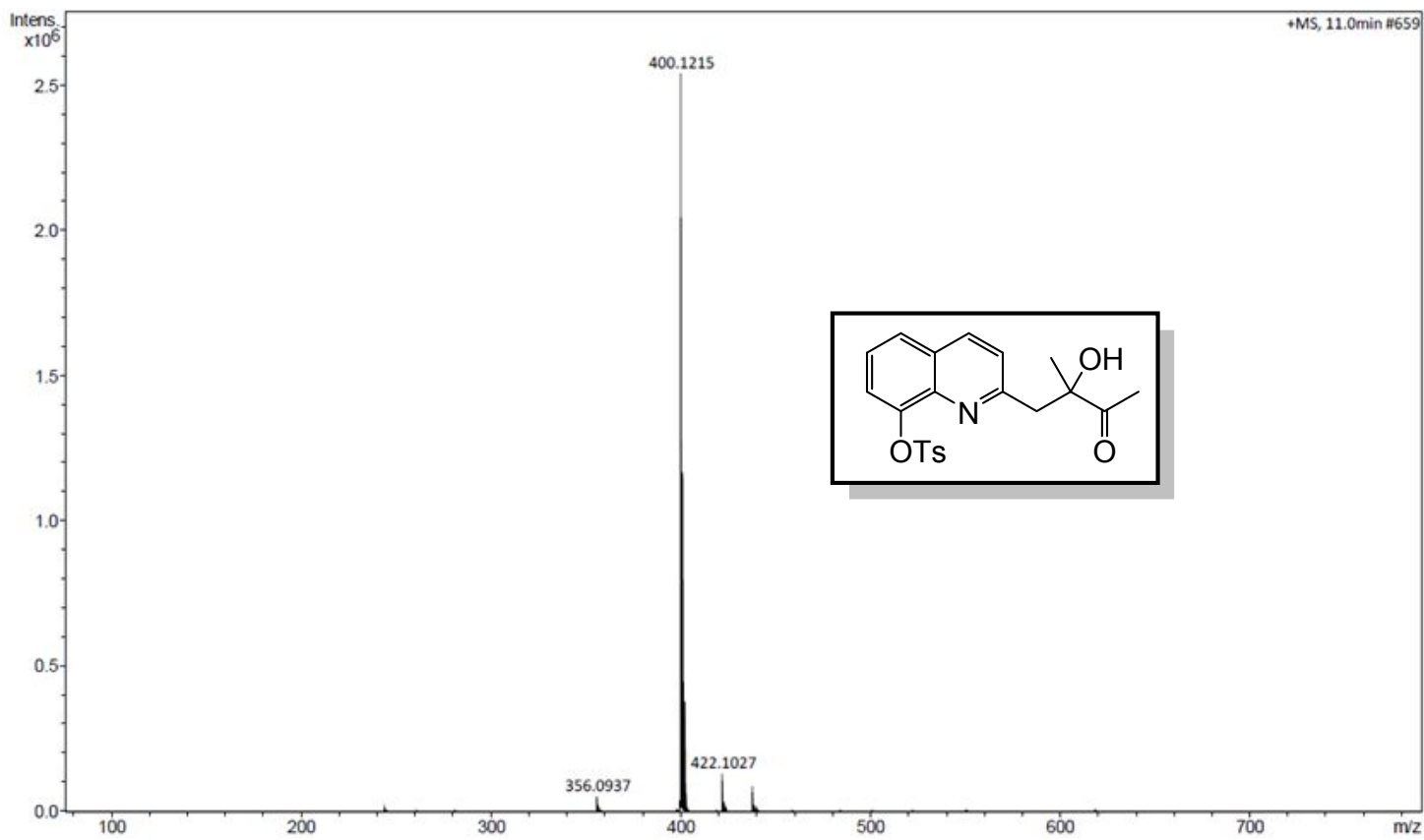

Figure S 11: 2-(2-hydroxy-2-methyl-3-oxobutyl)quinolin-8-yl 4-methylbenzenesulfonate (3ja)

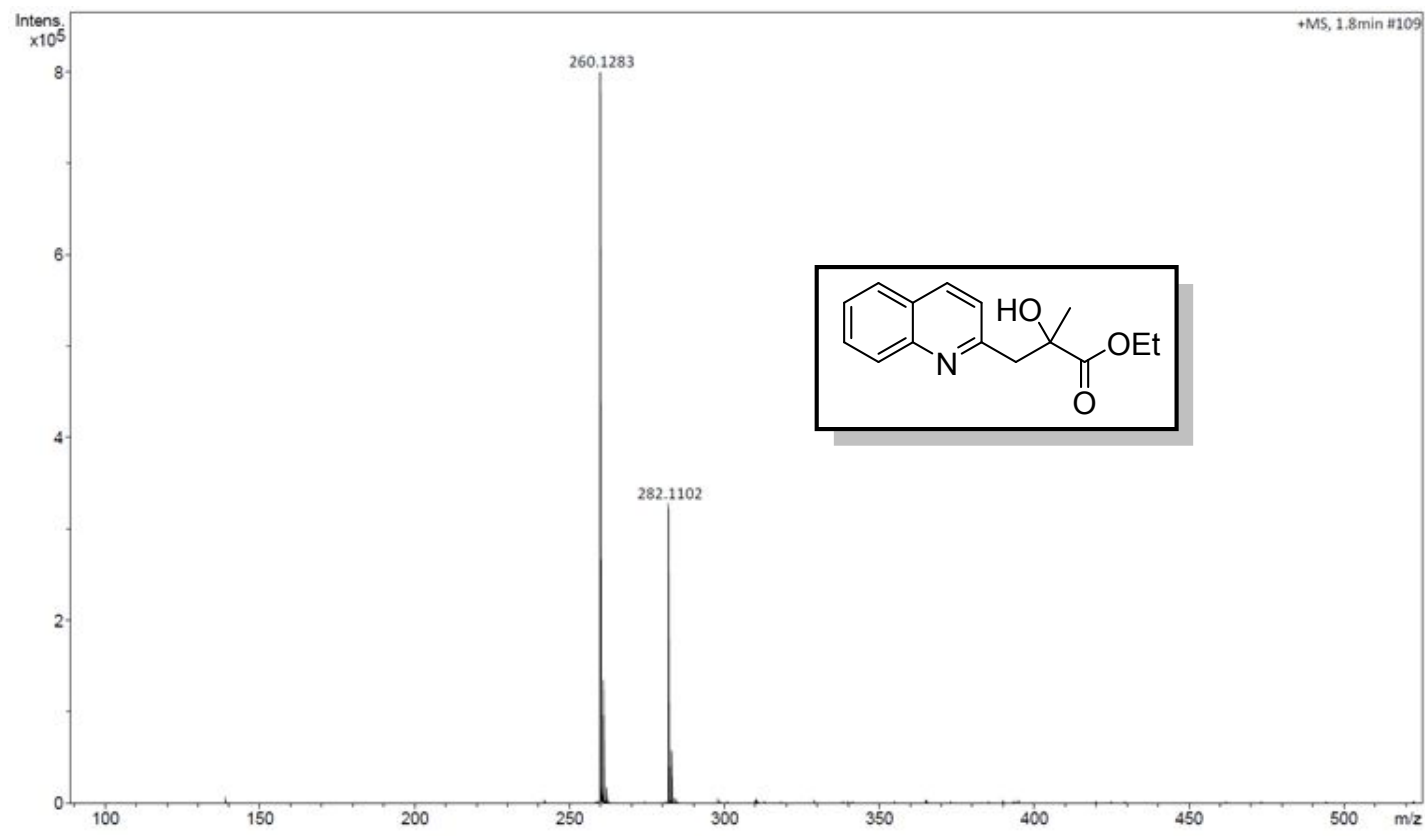

Figure S 12: ethyl 2-hydroxy-2-methyl-3-(quinolin-2-yl)propanoate (3ab) 


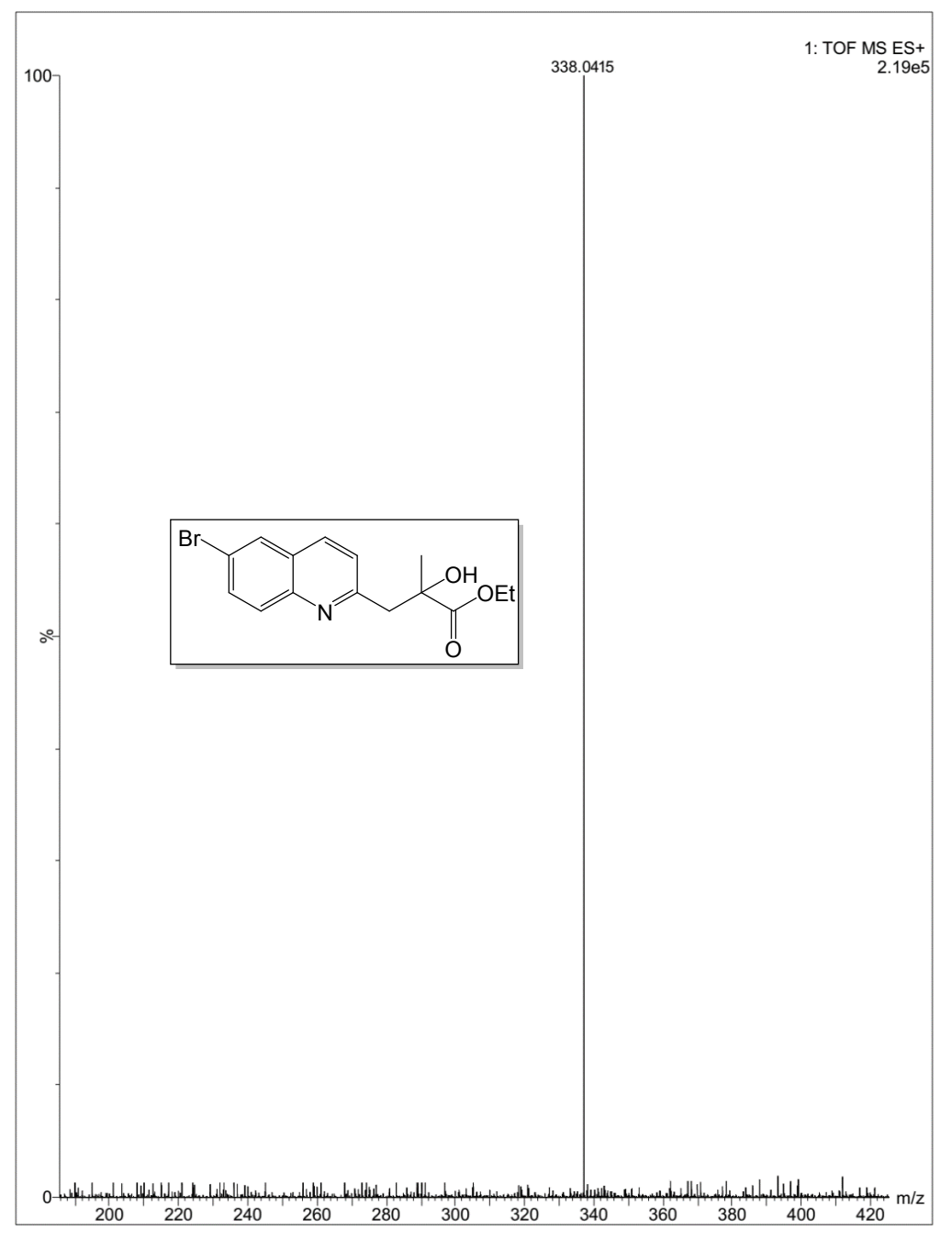

Figure S 13: Ethyl 3-(6-bromoquinolin-2-yl)-2-hydroxy-2-methylpropanoate (3bb) 


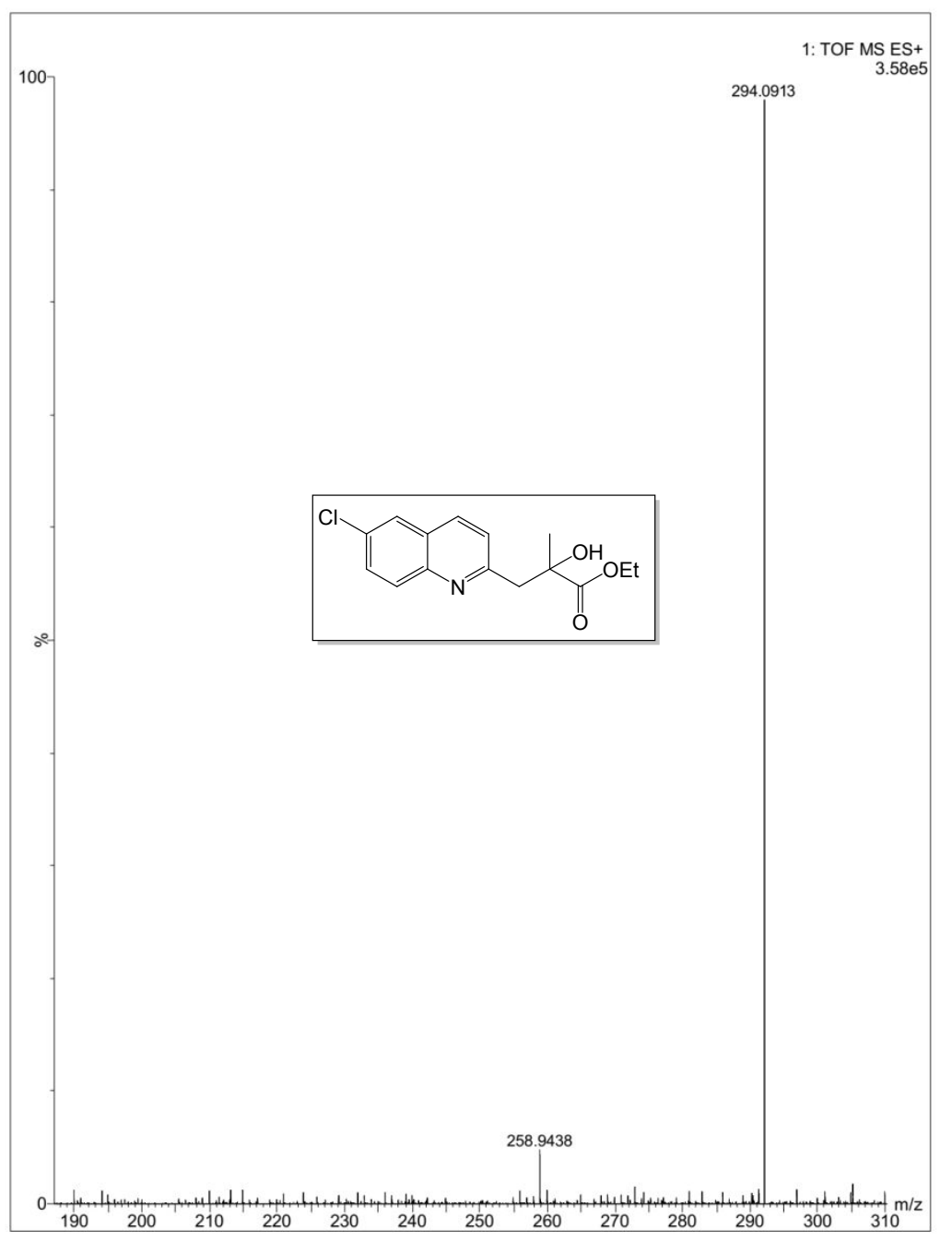

Figure S 14: ethyl 2-hydroxy-2-methyl-3-(6-chloroquinolin-2-yl)propanoate (3cb) 


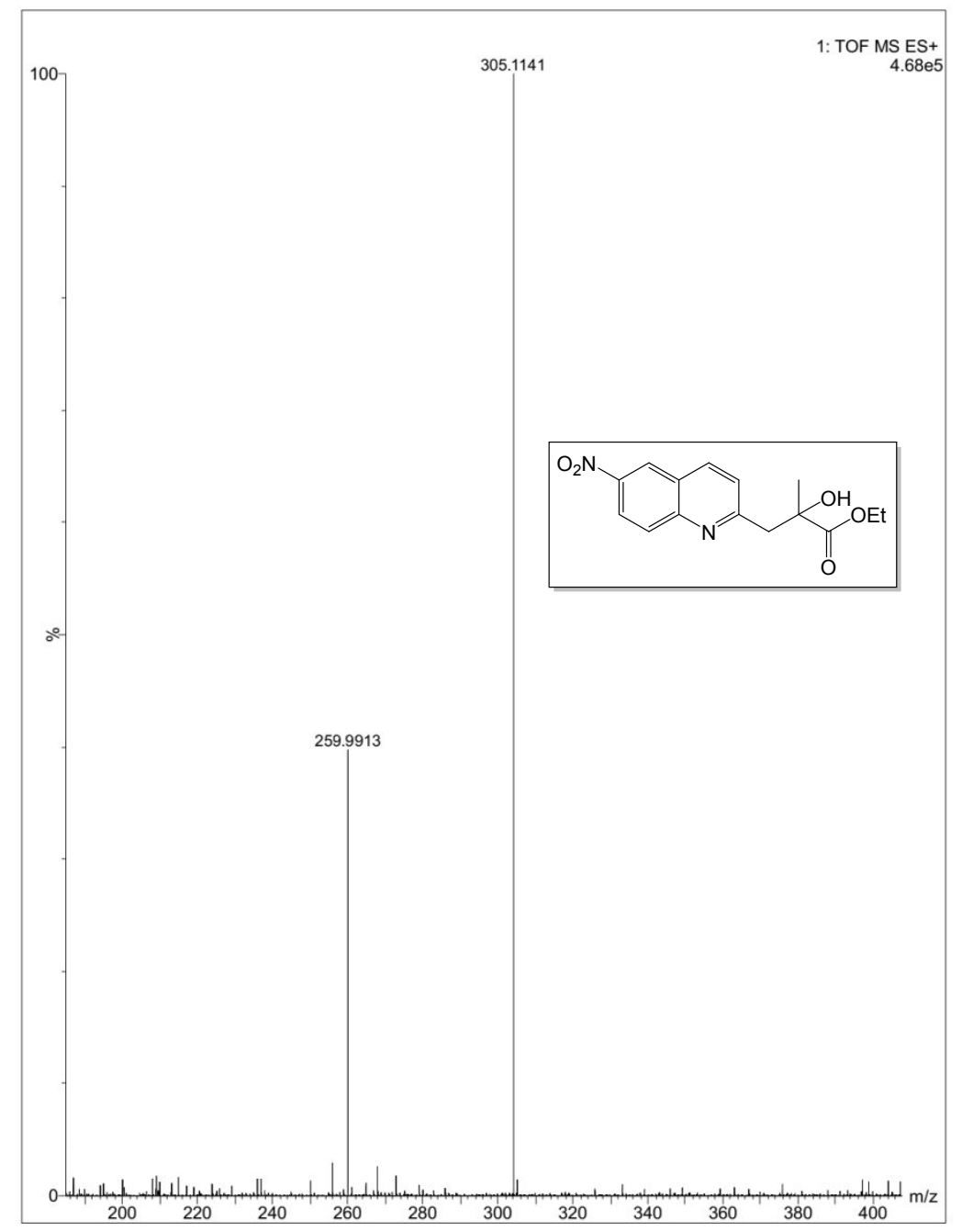

Figure S 15: 2-hydroxy-2-methyl-3-(6-nitroquinolin-2-yl)propanoate (3db) 


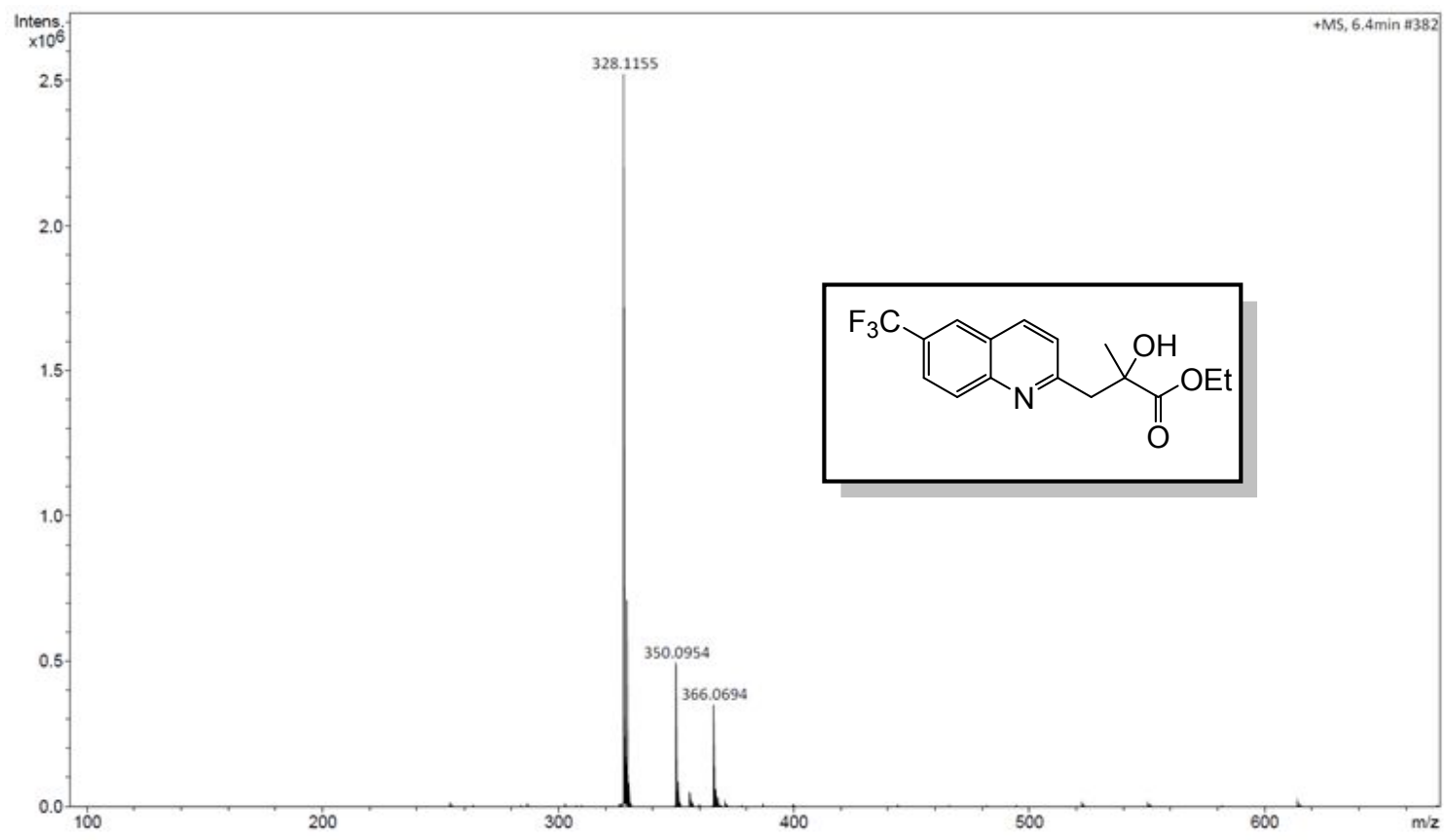

Figure S 16: ethyl 2-hydroxy-2-methyl-3-(6-(trifluoromethyl)quinolin-2-yl)propanoate (3eb) 


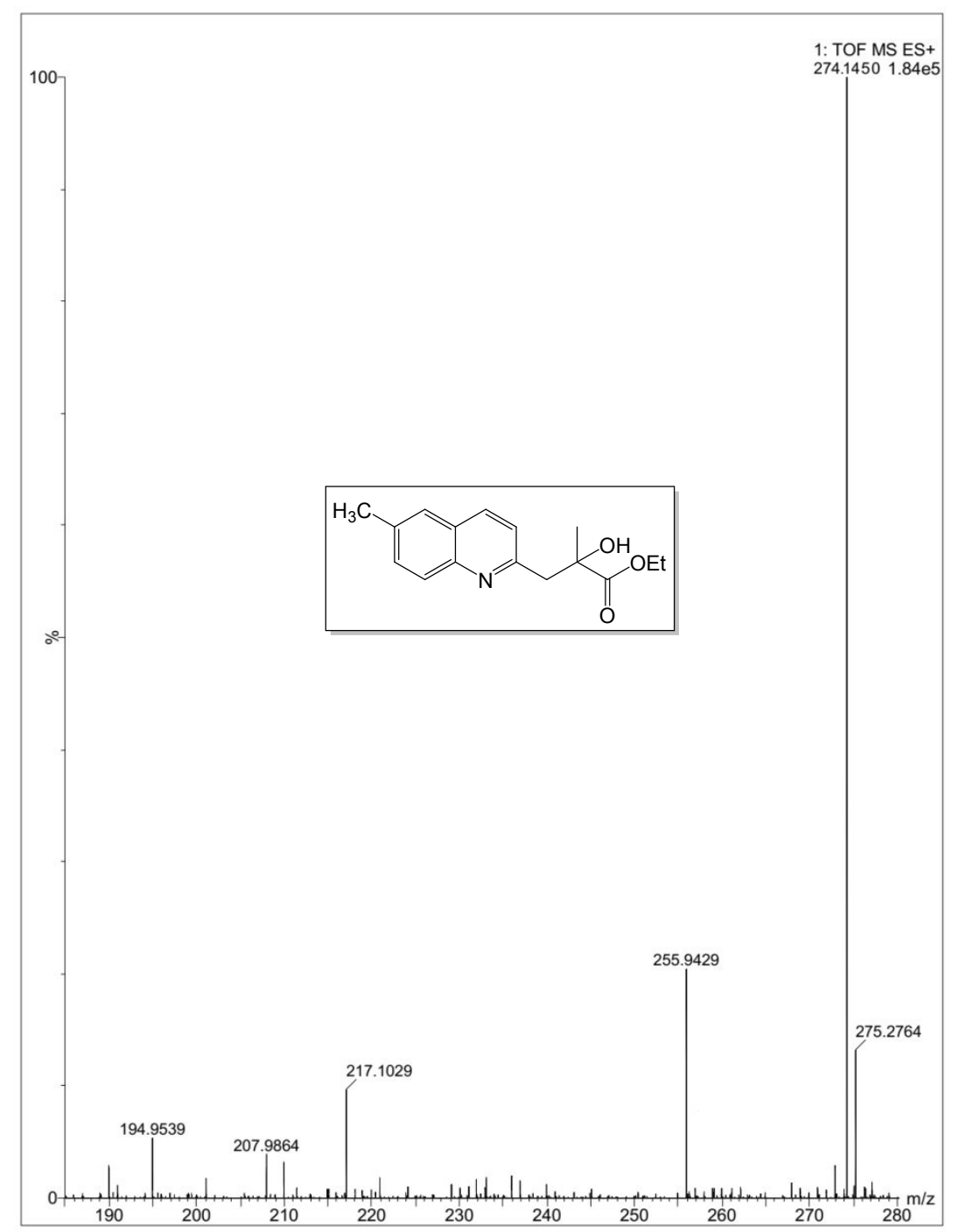

Figure S 17: 2-hydroxy-2-methyl-3-(6-methylquinolin-2-yl)propanoate (3gb) 


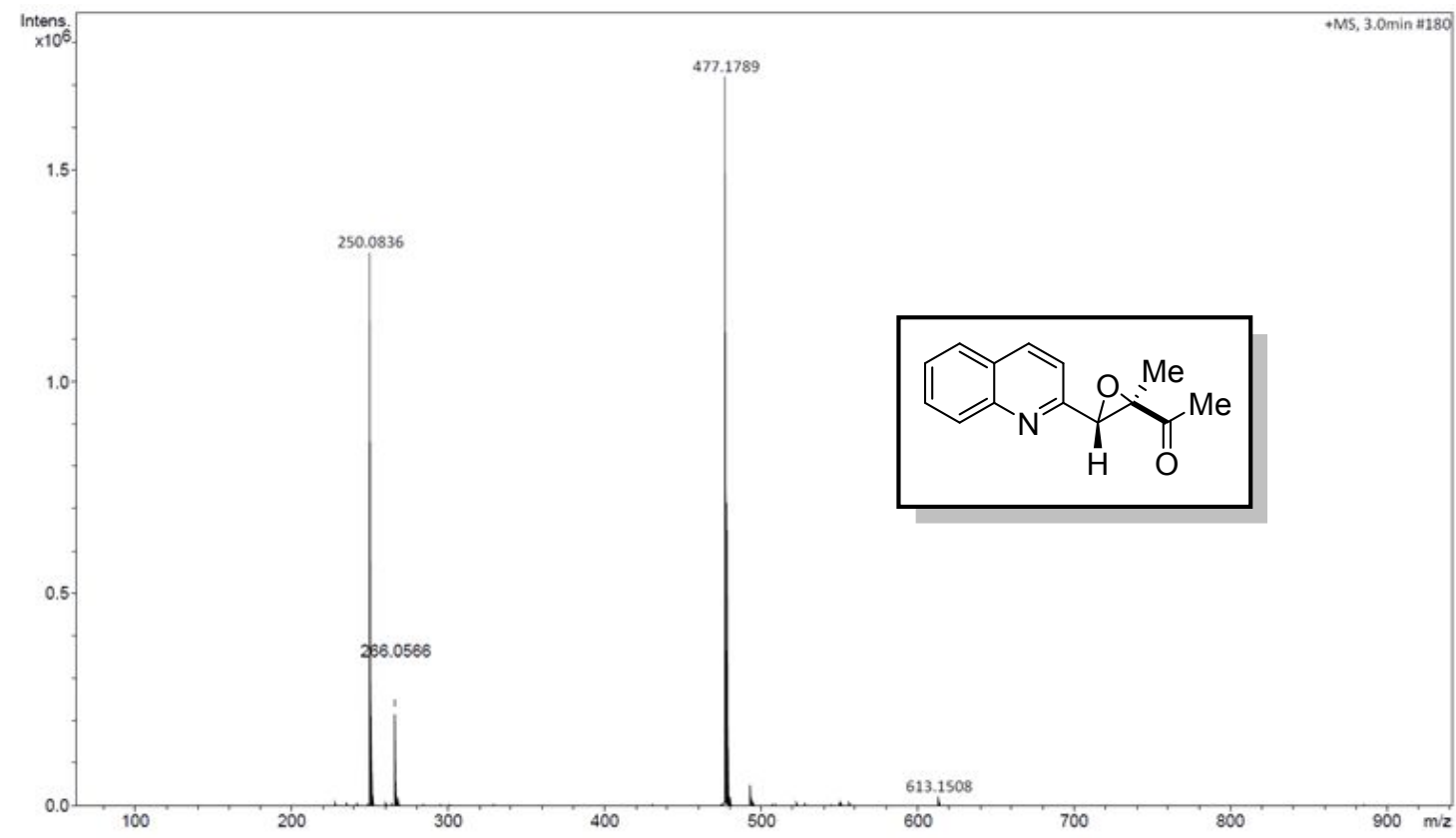

Figure S 18: 1-(2-methyl-3-(quinolin-2-yl)oxiran-2-yl)ethan-1-one (5)

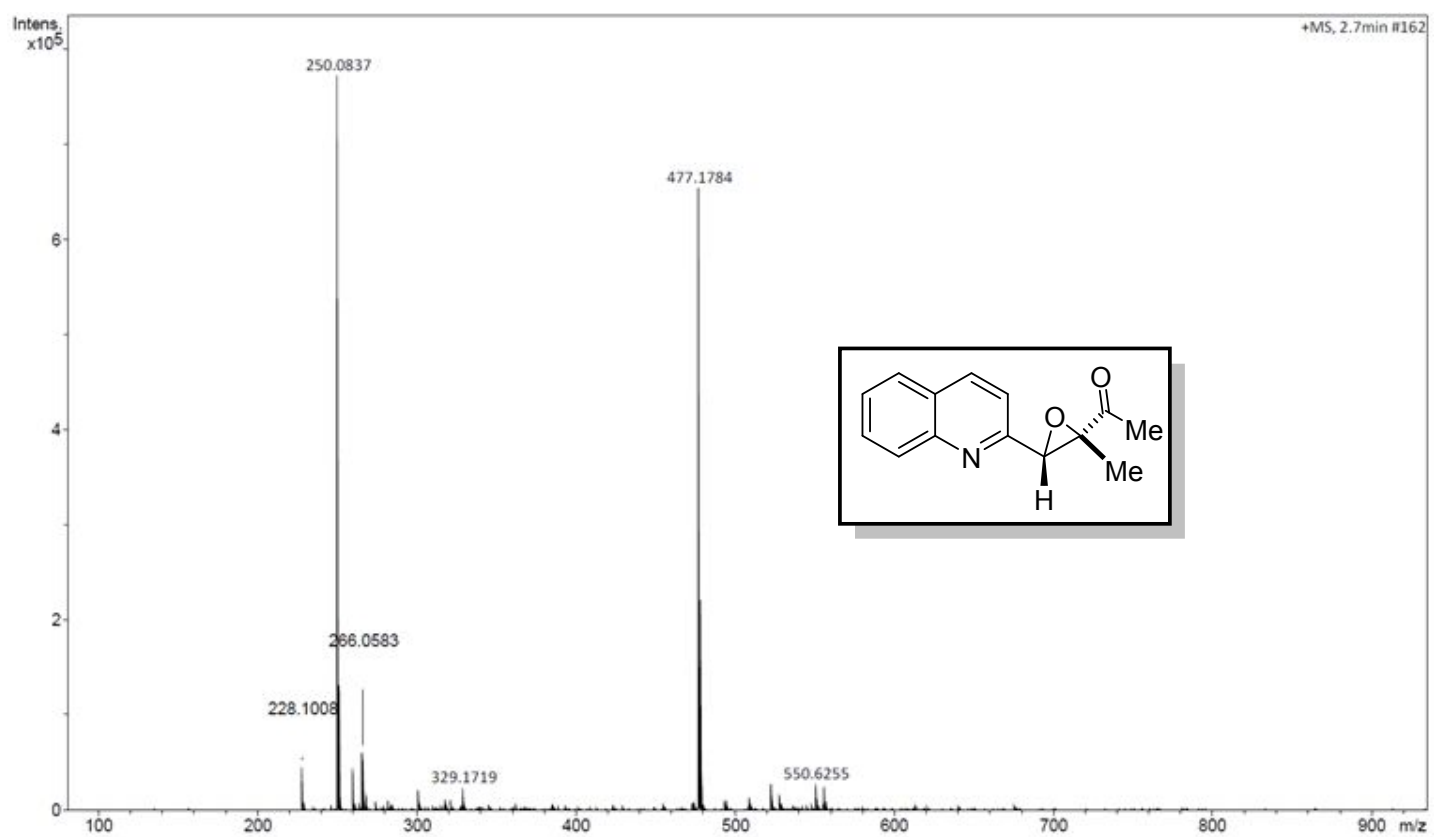

Figure S 19: 1-(2-methyl-3-(quinolin-2-yl)oxiran-2-yl)ethan-1-one (5') 
4. NMR Data $\left({ }^{1} \mathrm{H}\right.$ and ${ }^{13} \mathrm{C}$ spectra for all compounds in $\left.\mathrm{CDCl}_{3}\right)$

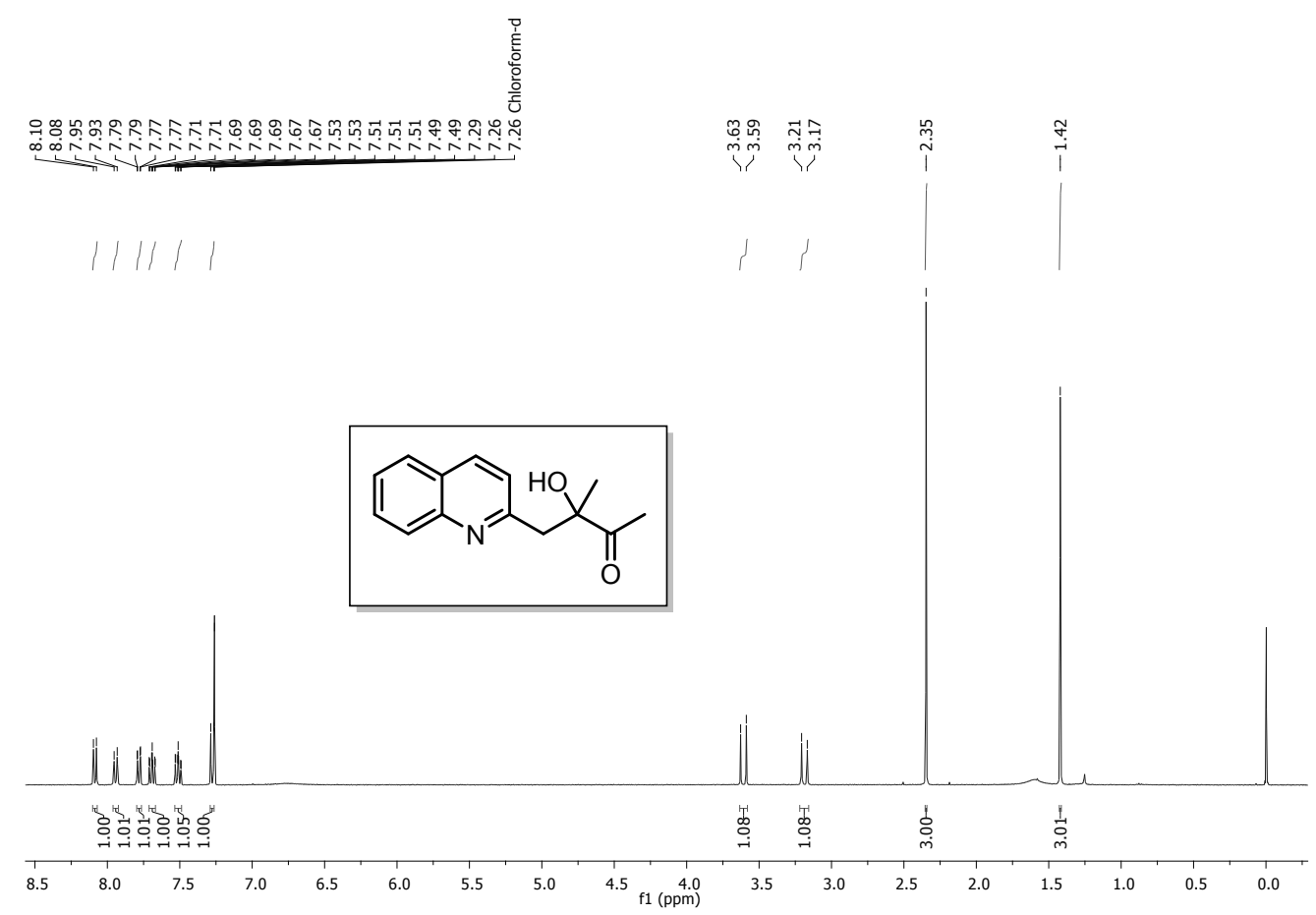

Figure S 20: ${ }^{1} \mathrm{H} \mathrm{NMR}\left(400 \mathrm{MHz}, \mathrm{CDCl}_{3}\right.$ ) of compound 3-hydroxy-3-methyl-4-(quinolin-2yl)butan-2-one (3aa) in $\mathrm{CDCl}_{3}$

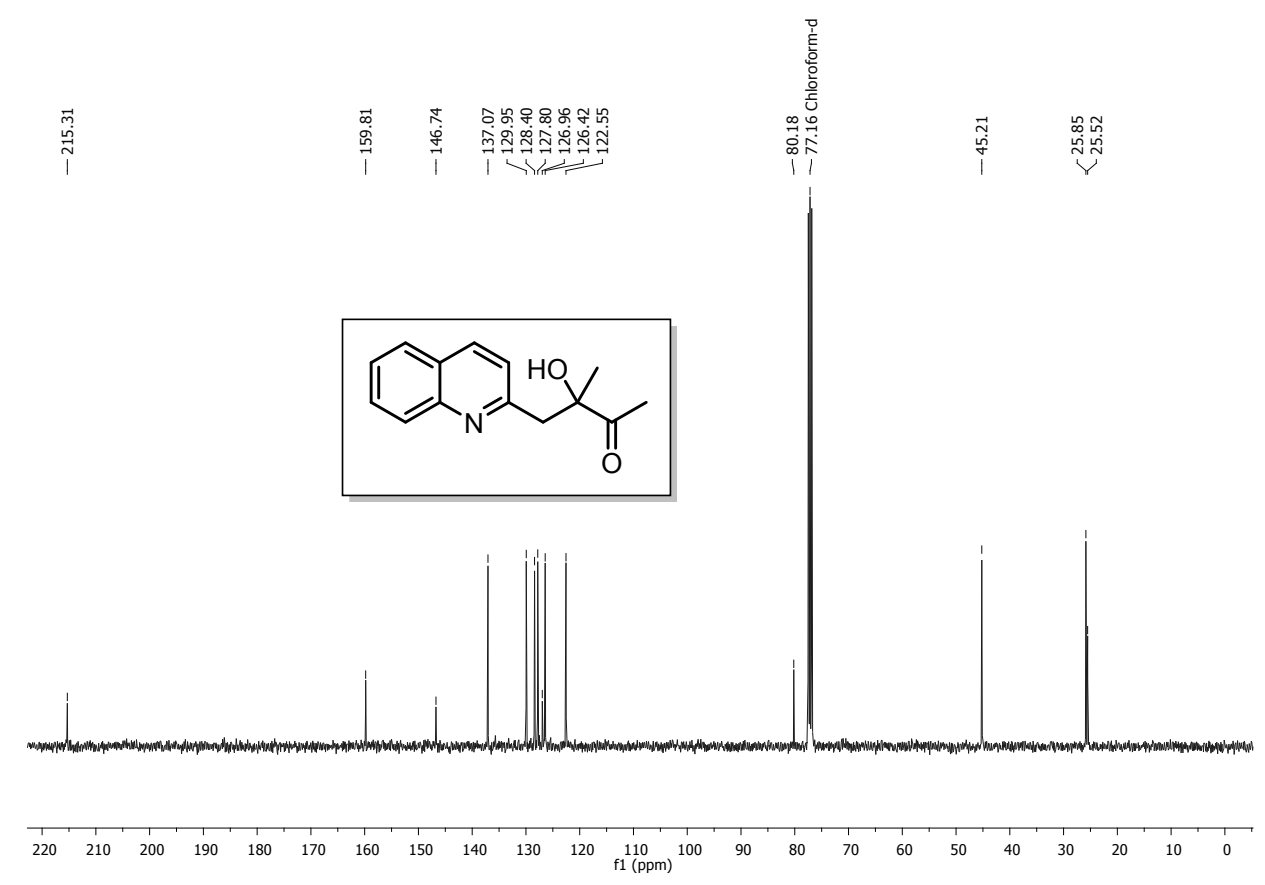

Figure $\mathrm{S} 21:{ }^{13} \mathrm{C} \mathrm{NMR}\left(101 \mathrm{MHz}, \mathrm{CDCl}_{3}\right.$ ) of the compound 3-hydroxy-3-methyl-4-(quinolin-2yl)butan-2-one (3aa) in $\mathrm{CDCl}_{3}$ 


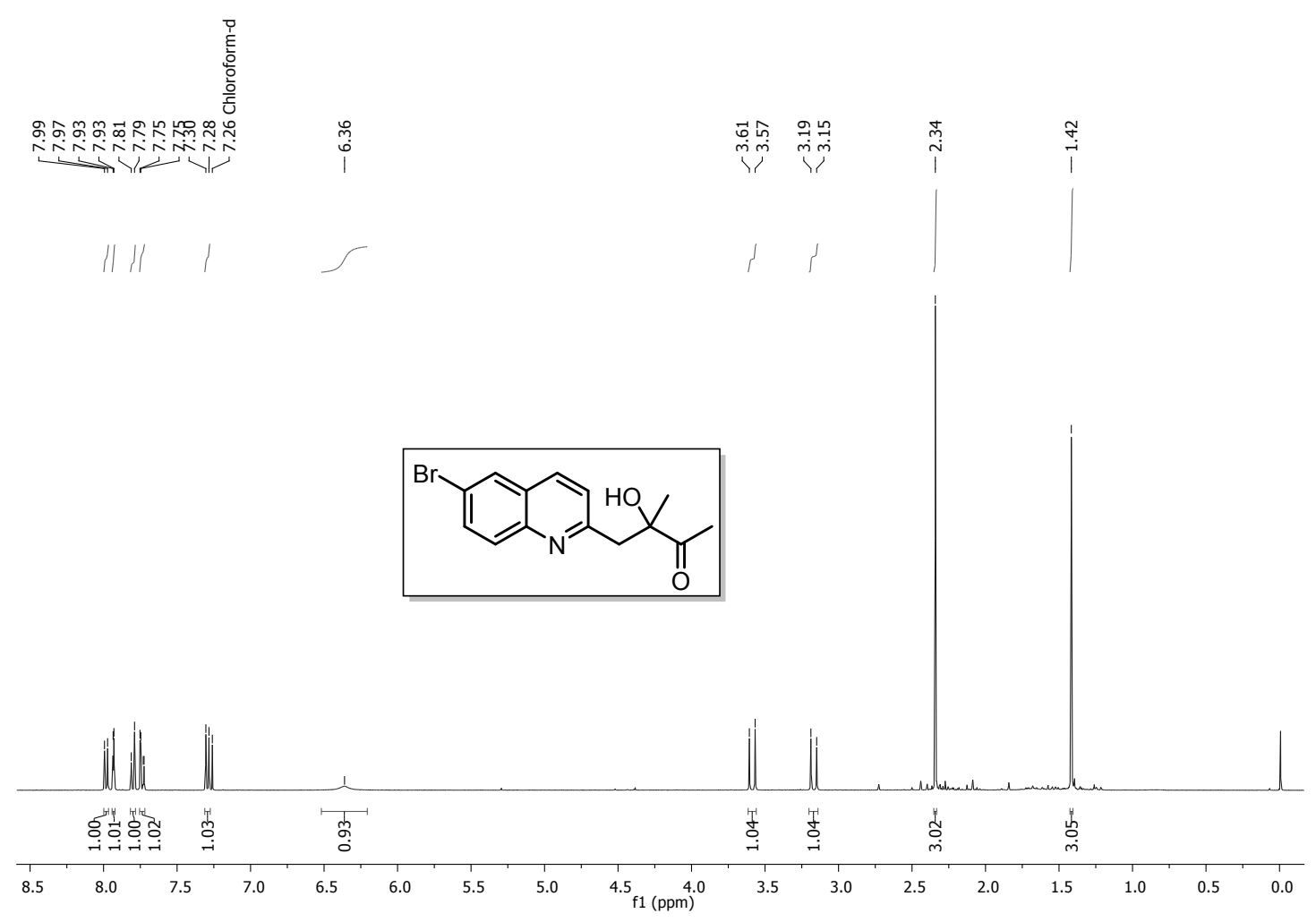

Figure $\mathrm{S} 22:{ }^{1} \mathrm{H}$ NMR (400 MHz, $\mathrm{CDCl}_{3}$ ) of the compound 4-(6-bromoquinolin-2-yl)-3hydroxy-3-methylbutan-2-one (3ba) in $\mathrm{CDCl}_{3}$

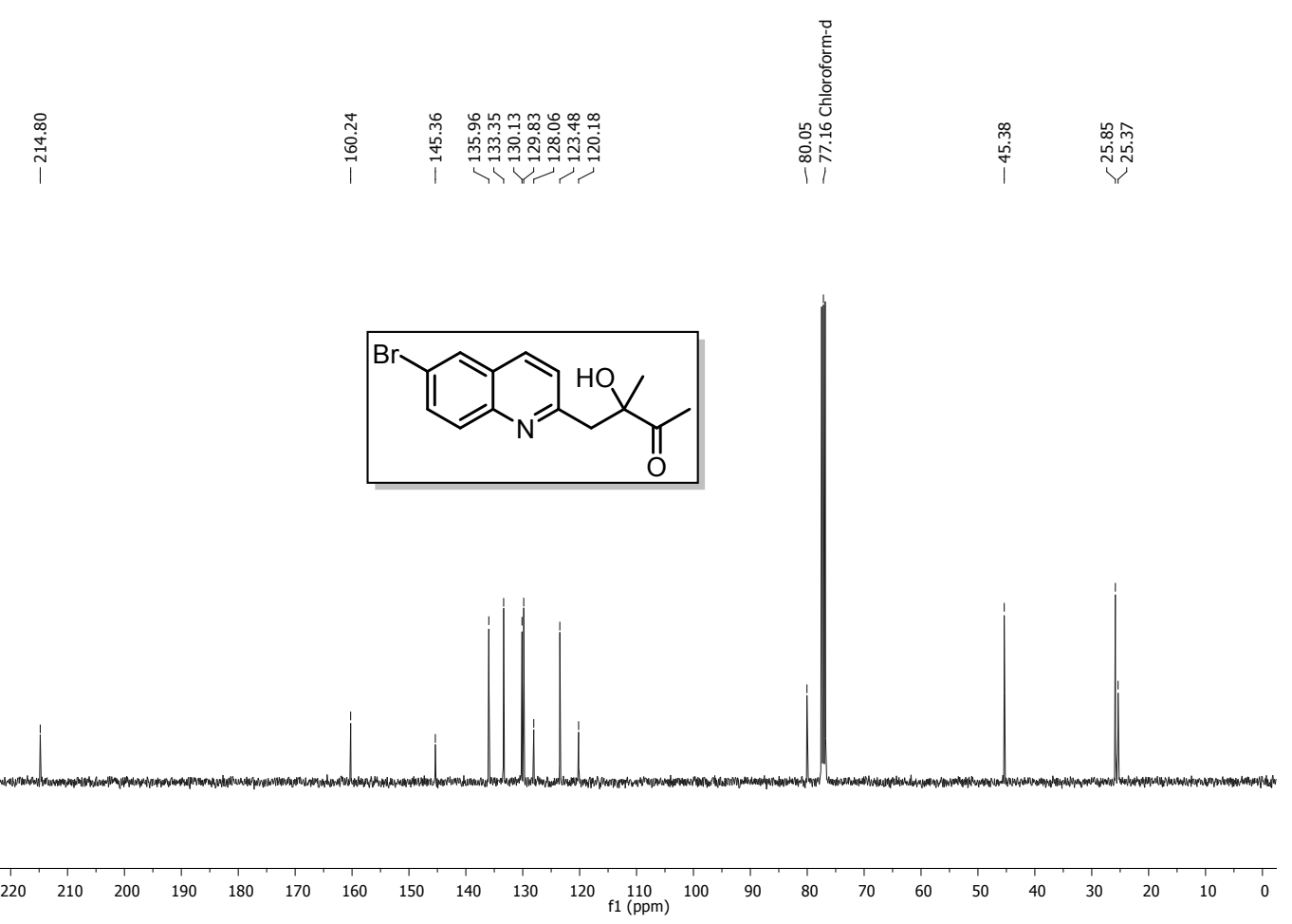

Figure S 23: ${ }^{13} \mathrm{C}$ NMR (101 MHz, $\mathrm{CDCl}_{3}$ ) of compound 4-(6-bromoquinolin-2-yl)-3-hydroxy3-methylbutan-2-one (3ba) in $\mathrm{CDCl}_{3}$ 


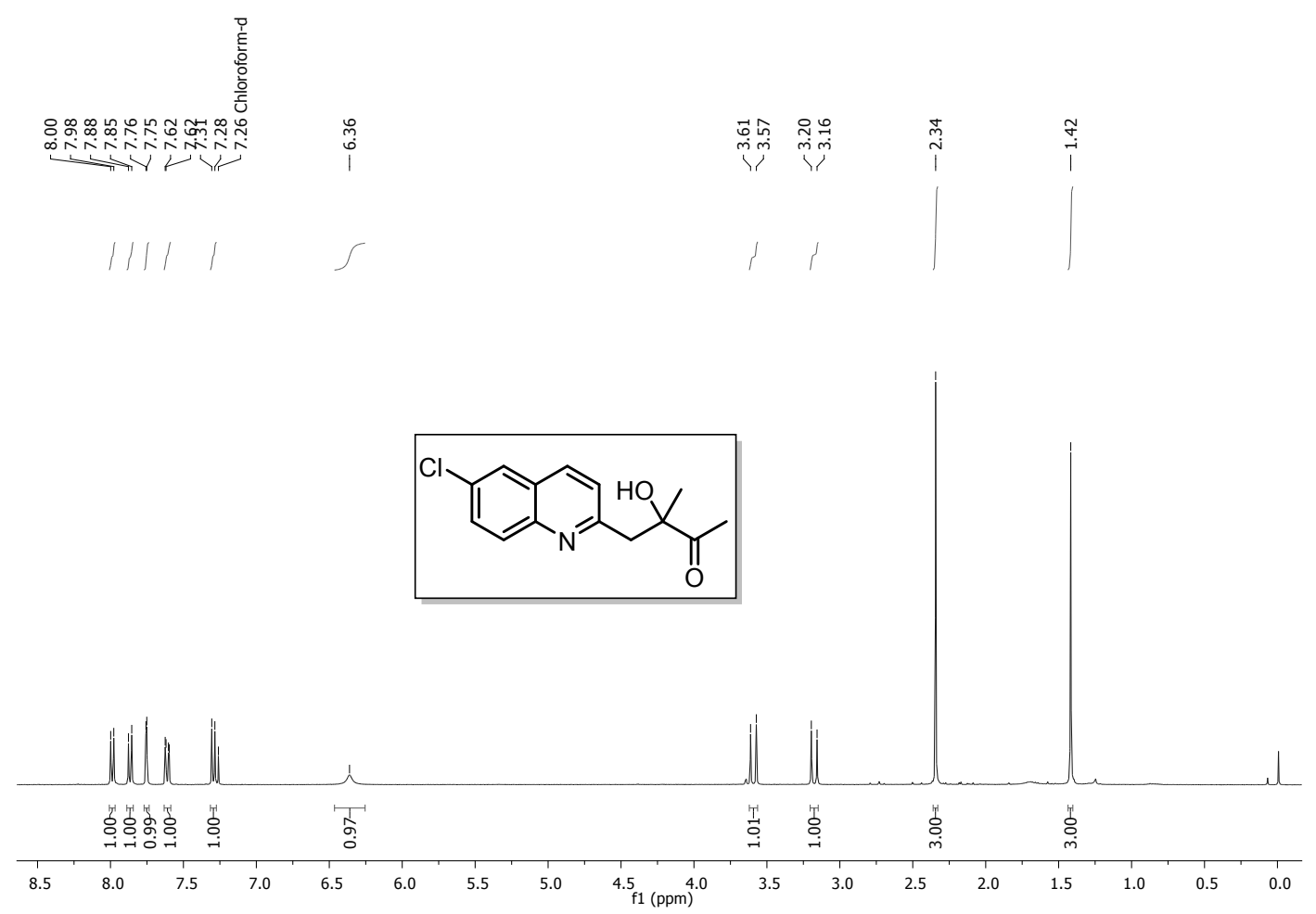

Figure S 24: ${ }^{1} \mathrm{H}$ NMR (400 MHz, $\mathrm{CDCl}_{3}$ ) of the compound 4-(6-chloroquinolin-2-yl)-3hydroxy-3-methylbutan-2-one (3ca) in $\mathrm{CDCl}_{3}$

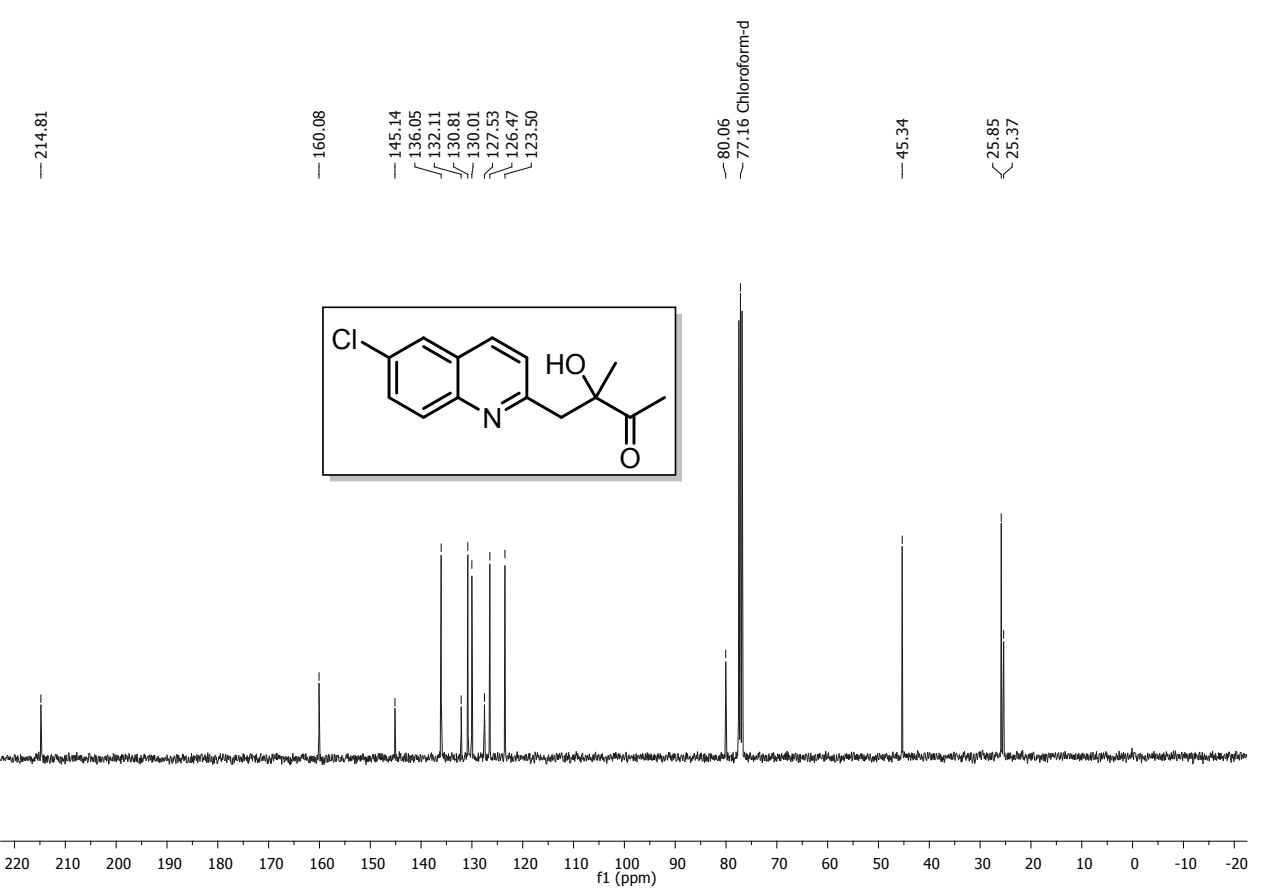

Figure $\mathrm{S} 25:{ }^{13} \mathrm{C}$ NMR (101 MHz, $\mathrm{CDCl}_{3}$ ) of the compound 4-(6-chloroquinolin-2-yl)-3hydroxy-3-methylbutan-2-one (3ca) in $\mathrm{CDCl}_{3}$ 


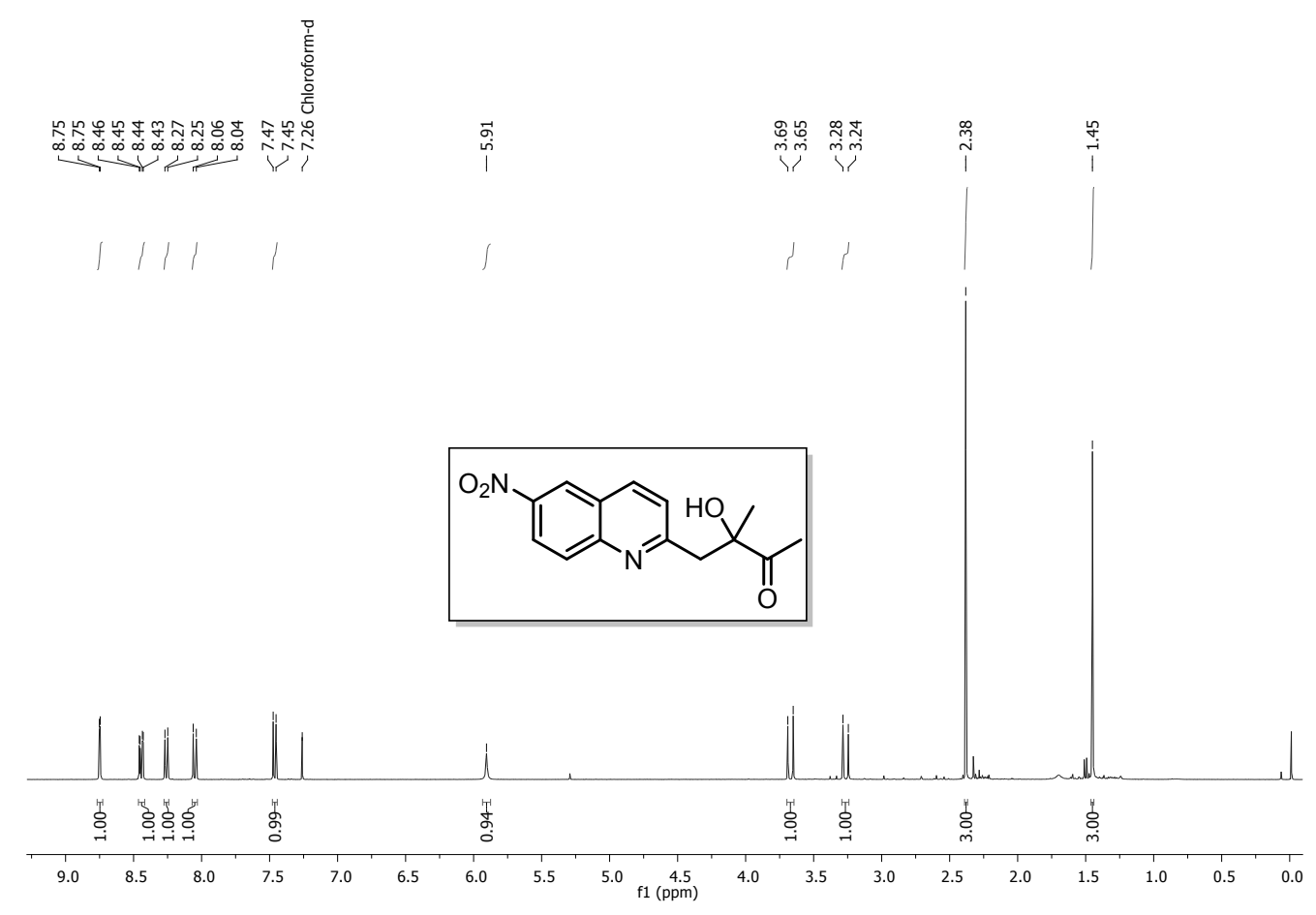

Figure S 26: ' $\mathrm{H}$ NMR (400 MHz, $\mathrm{CDCl}_{3}$ ) of compound 3-hydroxy-3-methyl-4-(6nitroquinolin-2-yl)butan-2-one (3da) in $\mathrm{CDCl}_{3}$
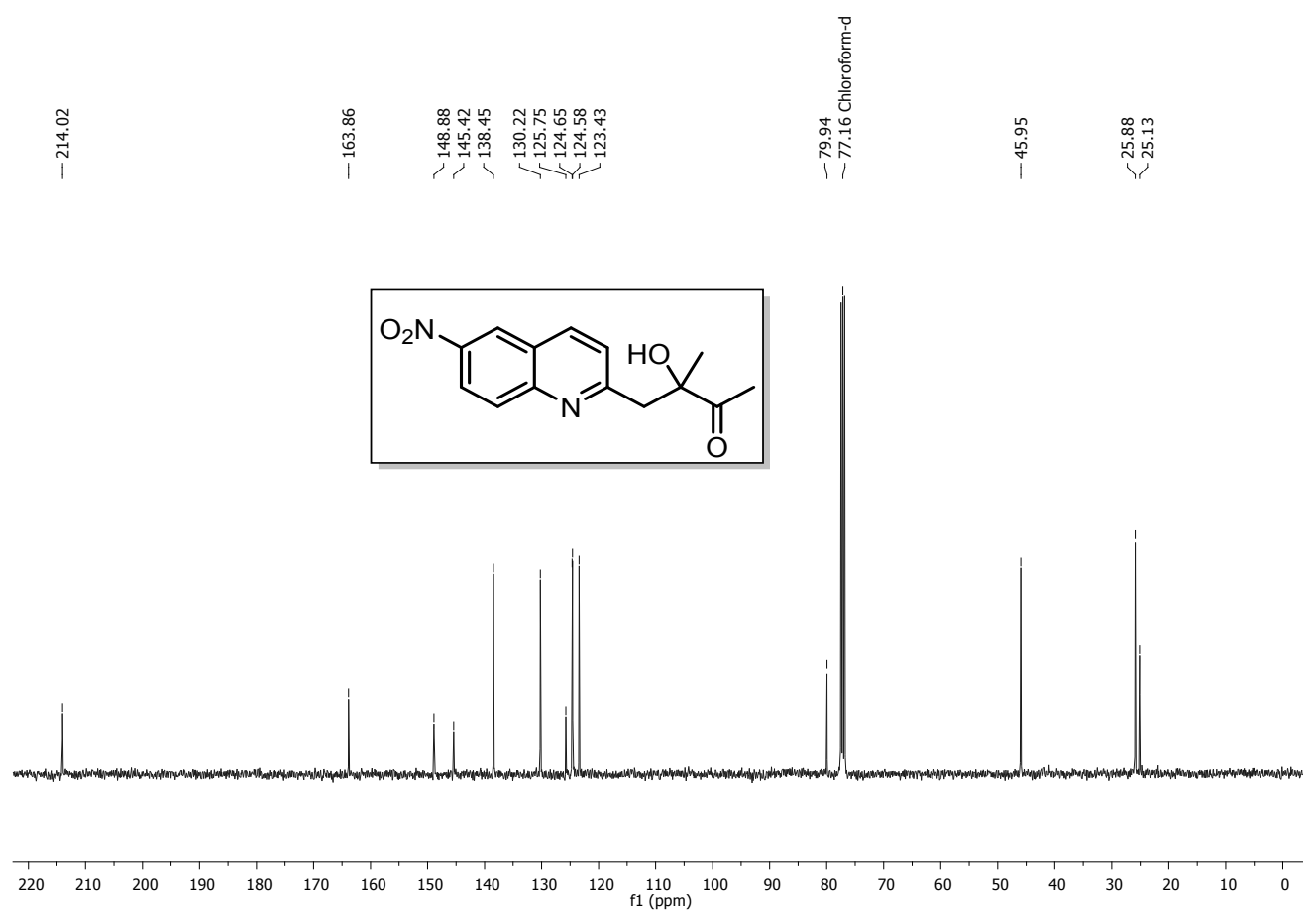

Figure $\mathrm{S} 27:{ }^{13} \mathrm{C} \mathrm{NMR}\left(101 \mathrm{MHz}, \mathrm{CDCl}_{3}\right.$ ) of compound 3-hydroxy-3-methyl-4-(6nitroquinolin-2-yl)butan-2-one (3da) in $\mathrm{CDCl}_{3}$ 


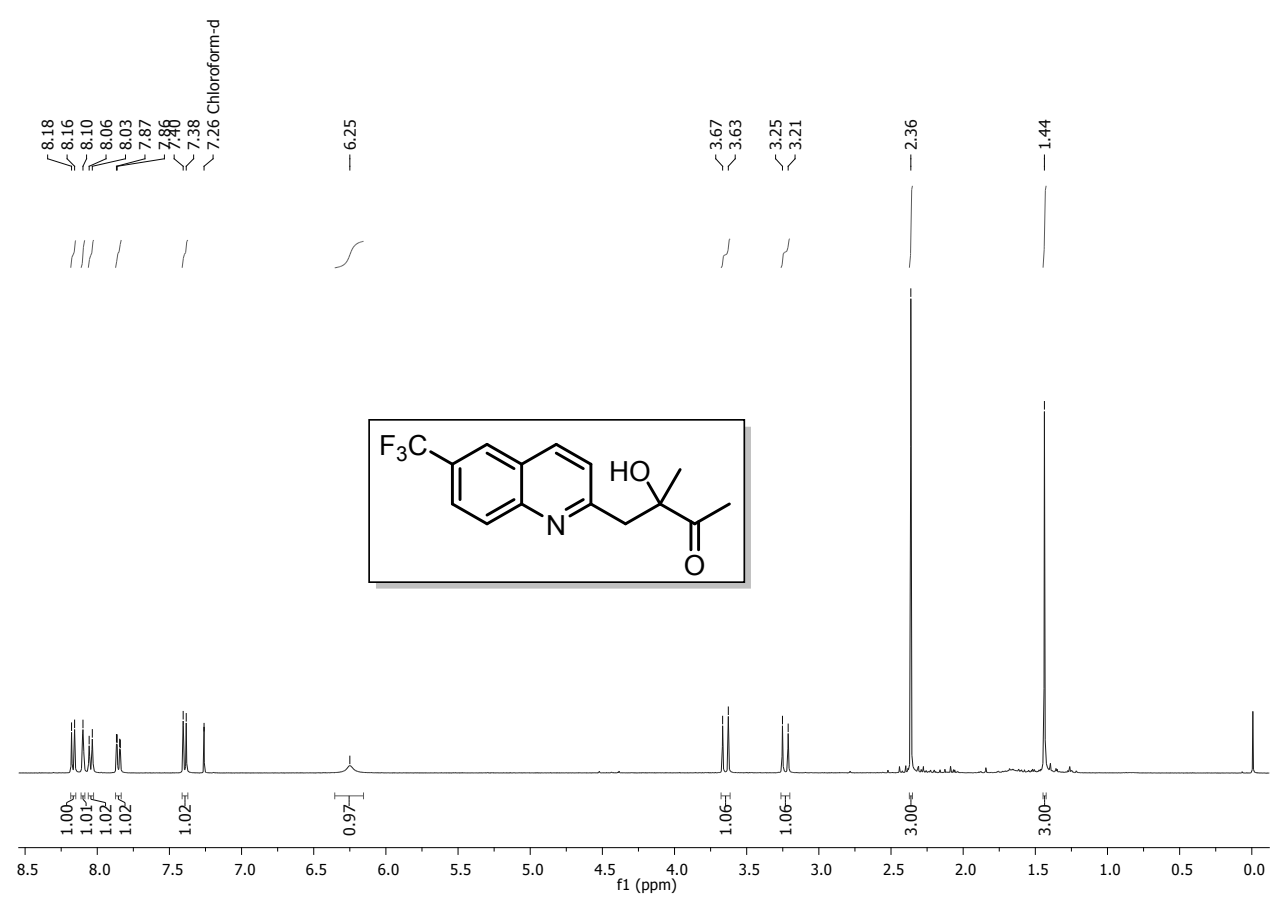

Figure $\mathrm{S} 28:{ }^{1} \mathrm{H}$ NMR (400 MHz, $\mathrm{CDCl}_{3}$ ) of compound 3-hydroxy-3-methyl-4-(6(trifluoromethyl)quinolin-2-yl)butan-2-one (3ea) in $\mathrm{CDCl}_{3}$
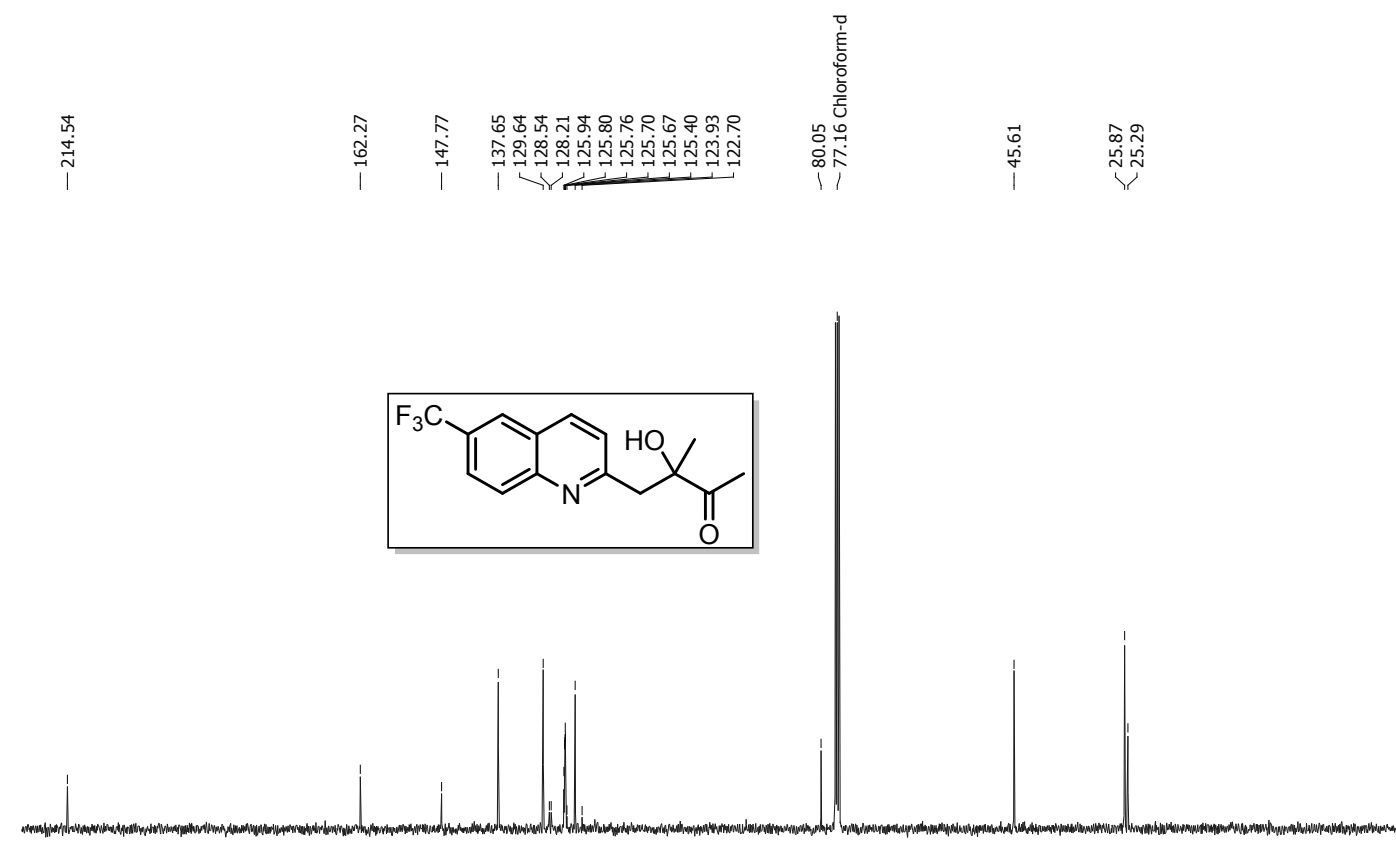

$\begin{array}{lllllllllllllllllllllllllllllllllllllll}220 & 210 & 200 & 190 & 180 & 170 & 160 & 150 & 140 & 130 & 120 & 110 & 100 & 90 & 80 & 70 & 60 & 50 & 40 & 30 & 20 & 10 & 0 & -10 & -20\end{array}$

Figure S 29: ${ }^{13} \mathrm{C}$ NMR (101 MHz, $\mathrm{CDCl}_{3}$ ) of compound 3-hydroxy-3-methyl-4-(6(trifluoromethyl)quinolin-2-yl)butan-2-one (3ea) in $\mathrm{CDCl}_{3}$ 


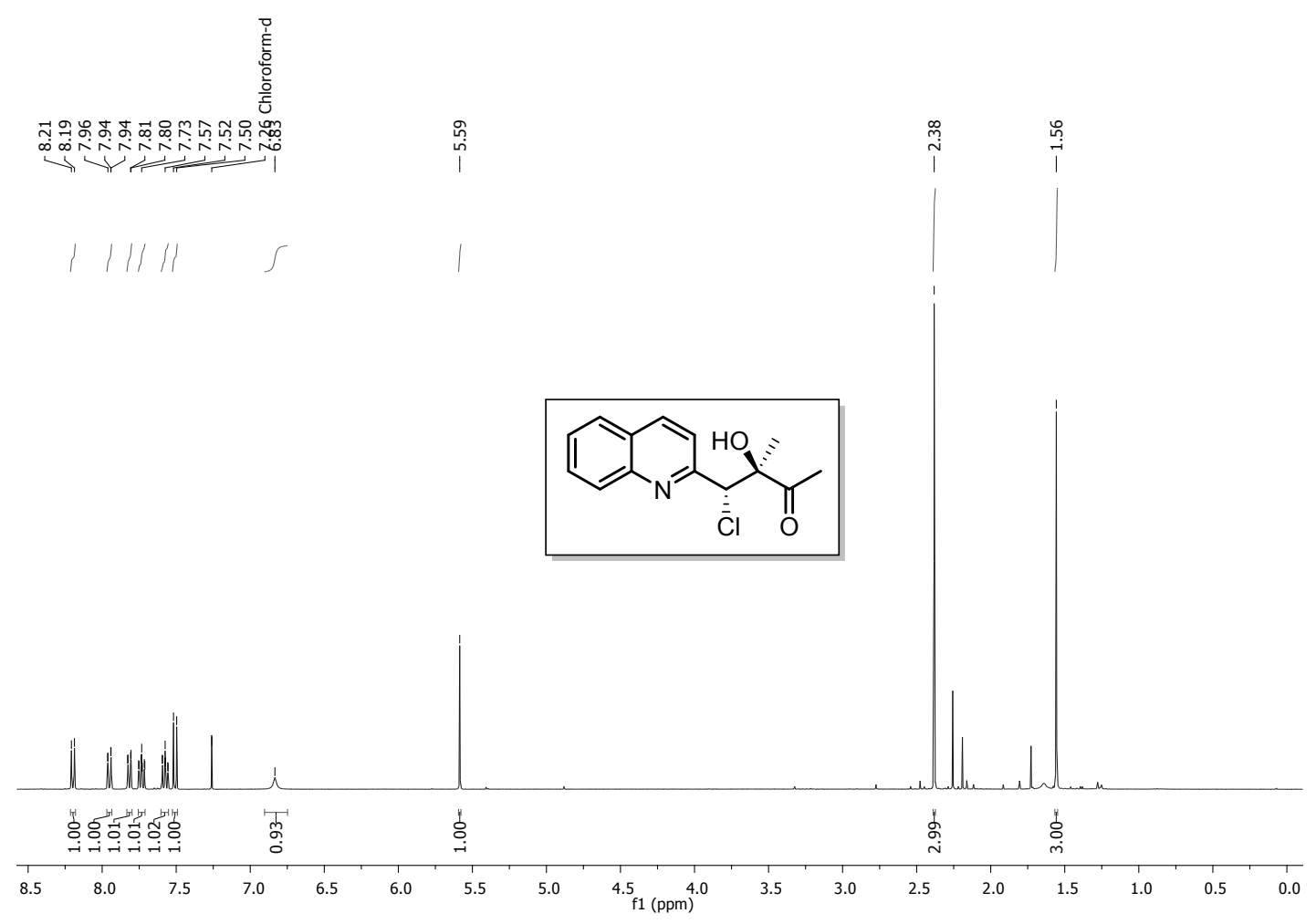

Figure S 30: ${ }^{1} \mathrm{H}$ NMR (400 MHz, $\mathrm{CDCl}_{3}$ ) of compound 4-chloro-3-hydroxy-3-methyl-4(quinolin-2-yl)butan-2-one (3fa) in $\mathrm{CDCl}_{3}$
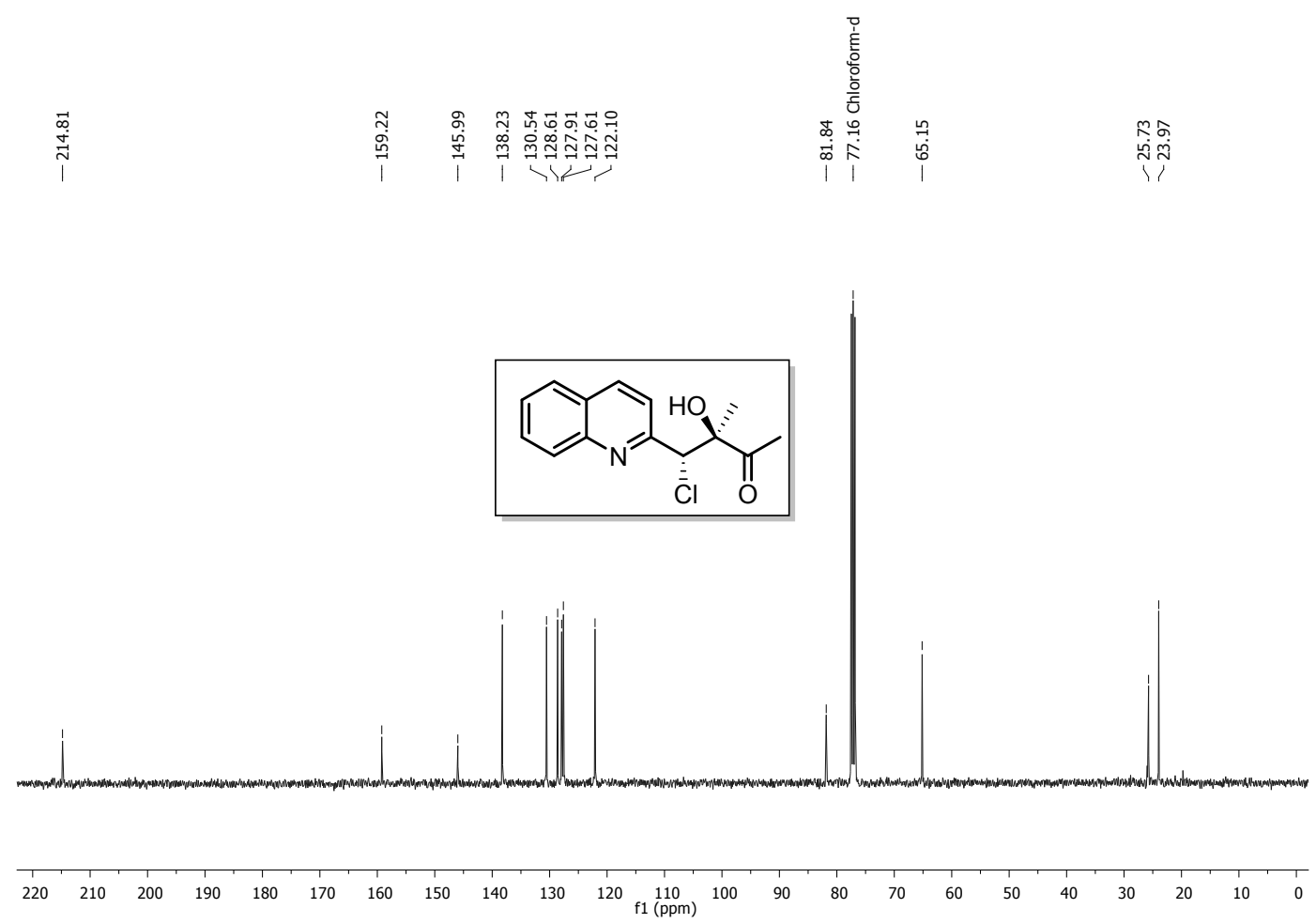

Figure $\mathrm{S} 31:{ }^{13} \mathrm{C} \mathrm{NMR}\left(101 \mathrm{MHz}, \mathrm{CDCl}_{3}\right.$ ) of compound 4-chloro-3-hydroxy-3-methyl-4(quinolin-2-yl)butan-2-one (3fa) in $\mathrm{CDCl}_{3}$ 


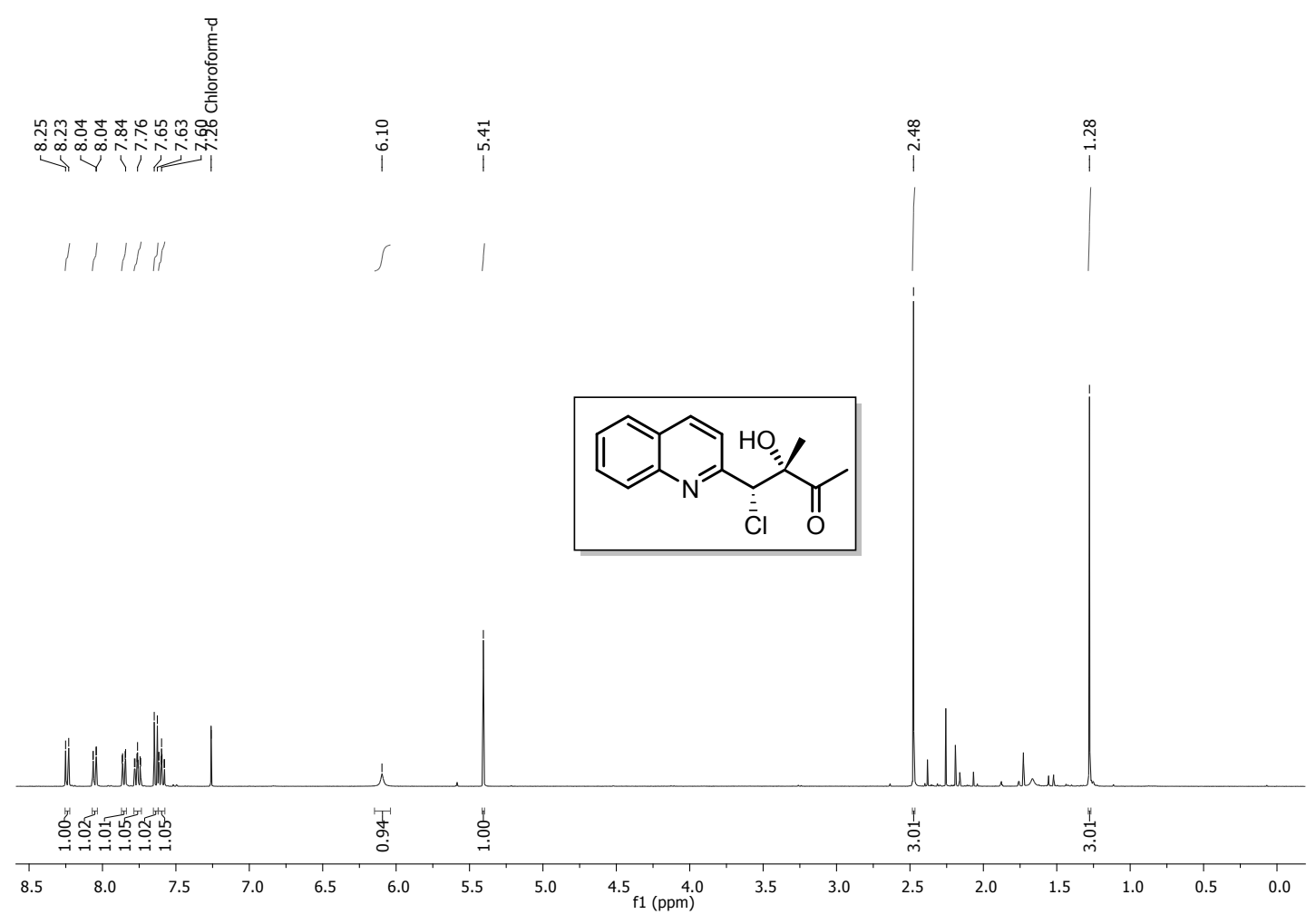

Figure S 32: ${ }^{1} \mathrm{H}$ NMR (400 MHz, $\mathrm{CDCl}_{3}$ ) of 4-chloro-3-hydroxy-3-methyl-4-(quinolin-2yl)butan-2-one (3fa') in $\mathrm{CDCl}_{3}$

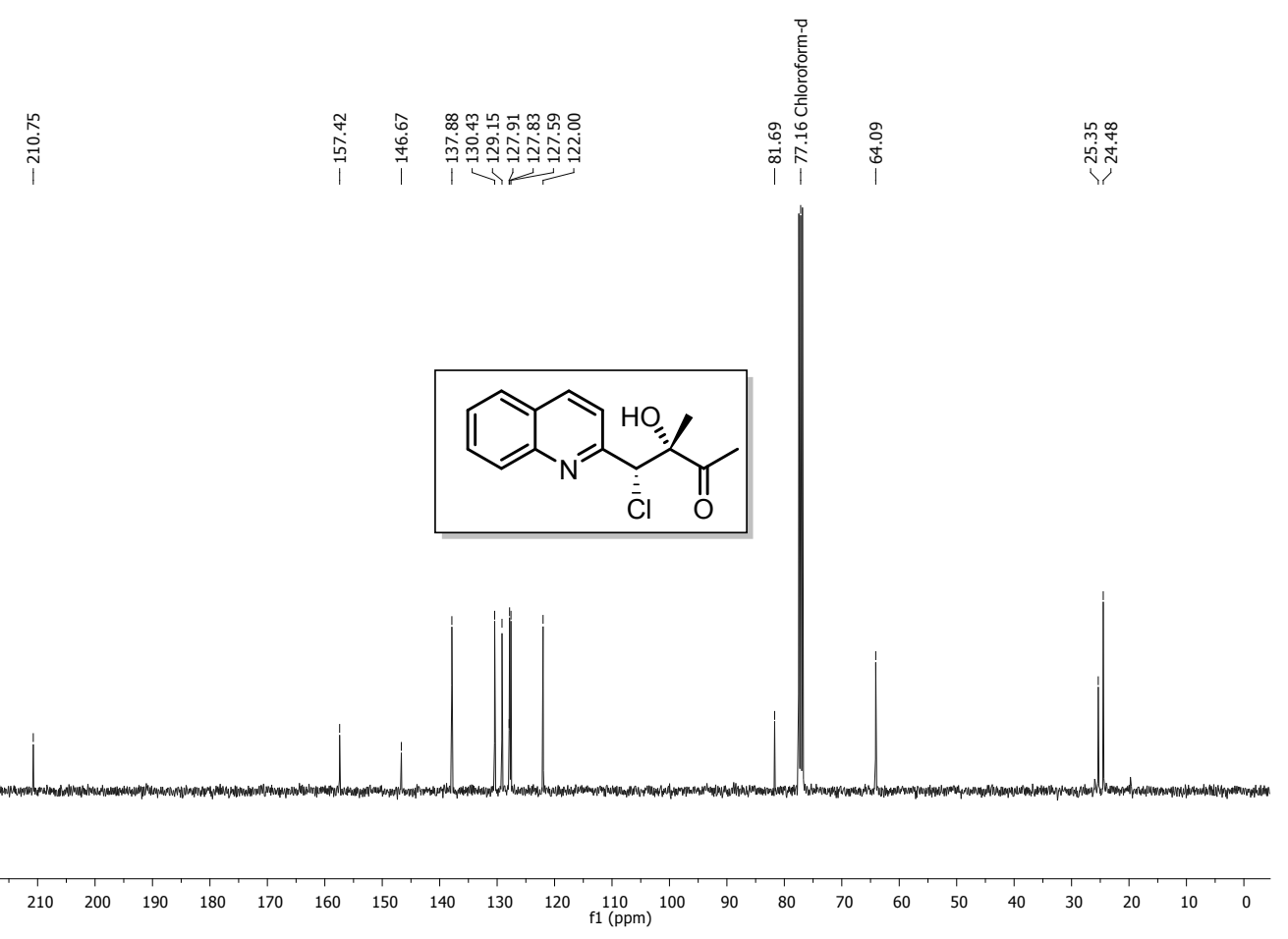

Figure S 33: ${ }^{13} \mathrm{C}$ NMR (101 MHz, $\mathrm{CDCl}_{3}$ ) of compound 4-chloro-3-hydroxy-3-methyl-4(quinolin-2-yl)butan-2-one (3fa') in $\mathrm{CDCl}_{3}$ 


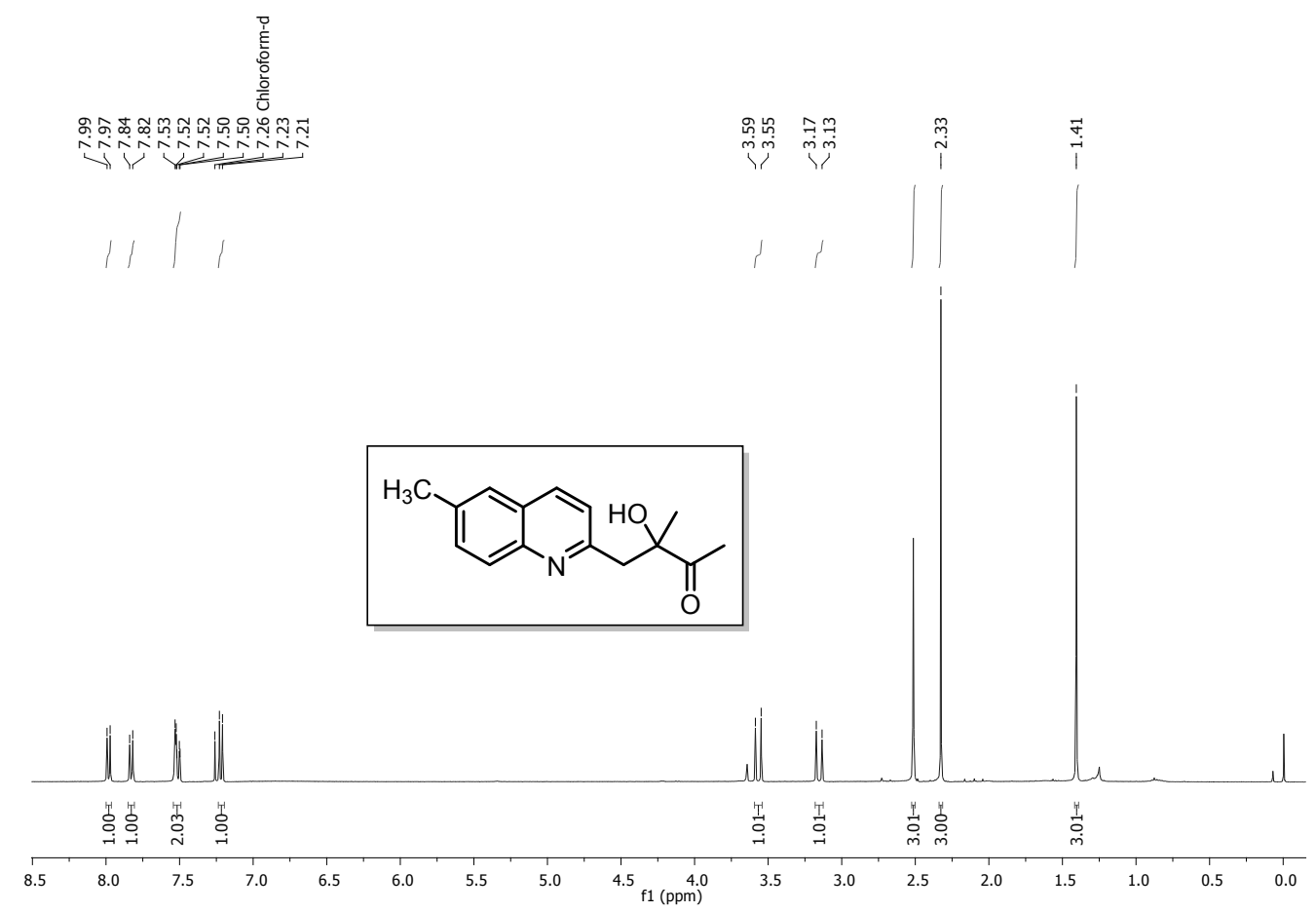

Figure S 34: ${ }^{1} \mathrm{H}$ NMR (400 MHz, $\mathrm{CDCl}_{3}$ ) of the compound 3-hydroxy-3-methyl-4-(6methylquinolin-2-yl)butan-2-one (3ga) in $\mathrm{CDCl}_{3}$

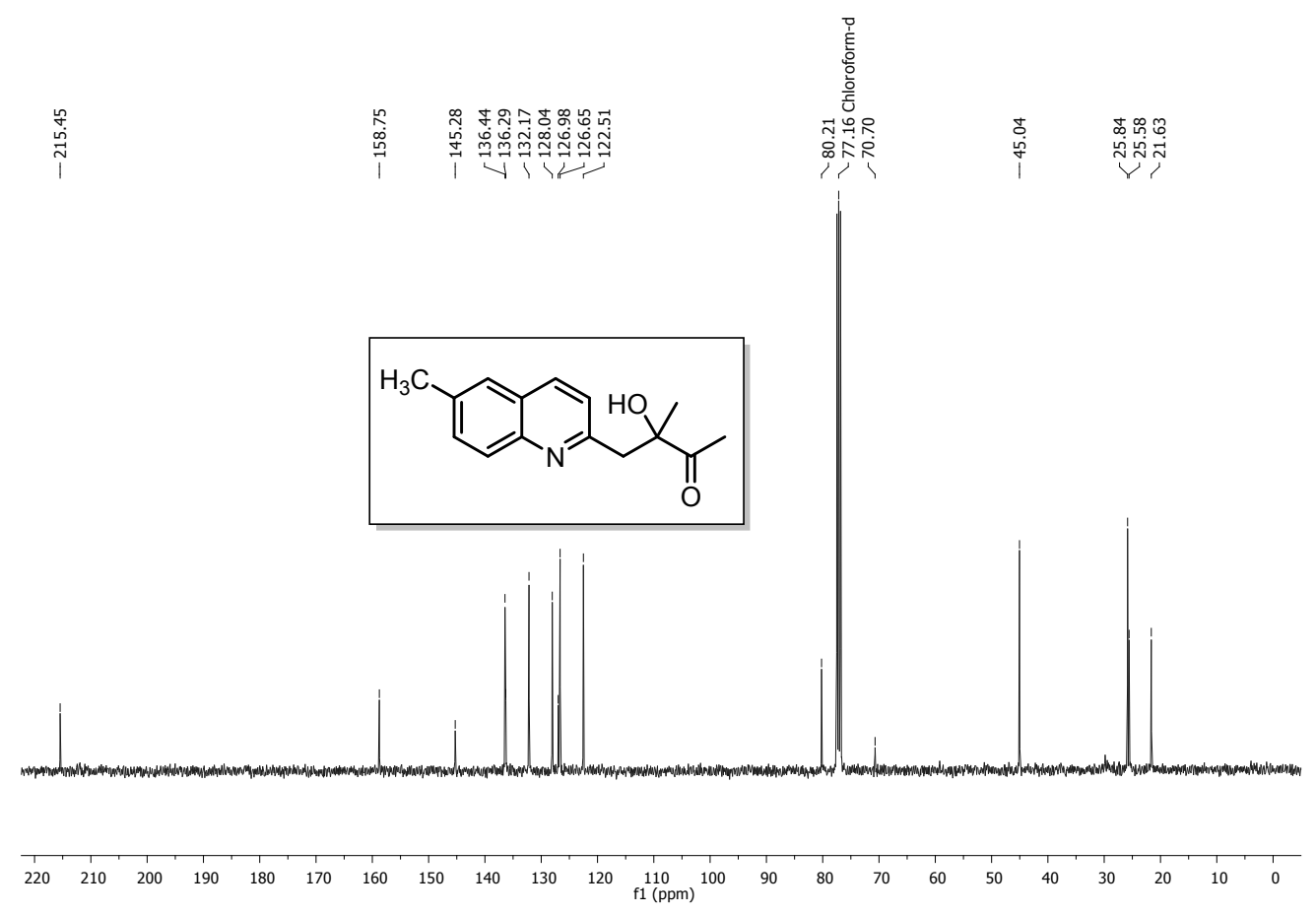

Figure $\mathrm{S} 35:{ }^{13} \mathrm{C}$ NMR (101 MHz, $\mathrm{CDCl}_{3}$ ) of the compound 3-hydroxy-3-methyl-4-(6methylquinolin-2-yl)butan-2-one (3ga) in $\mathrm{CDCl}_{3}$ 


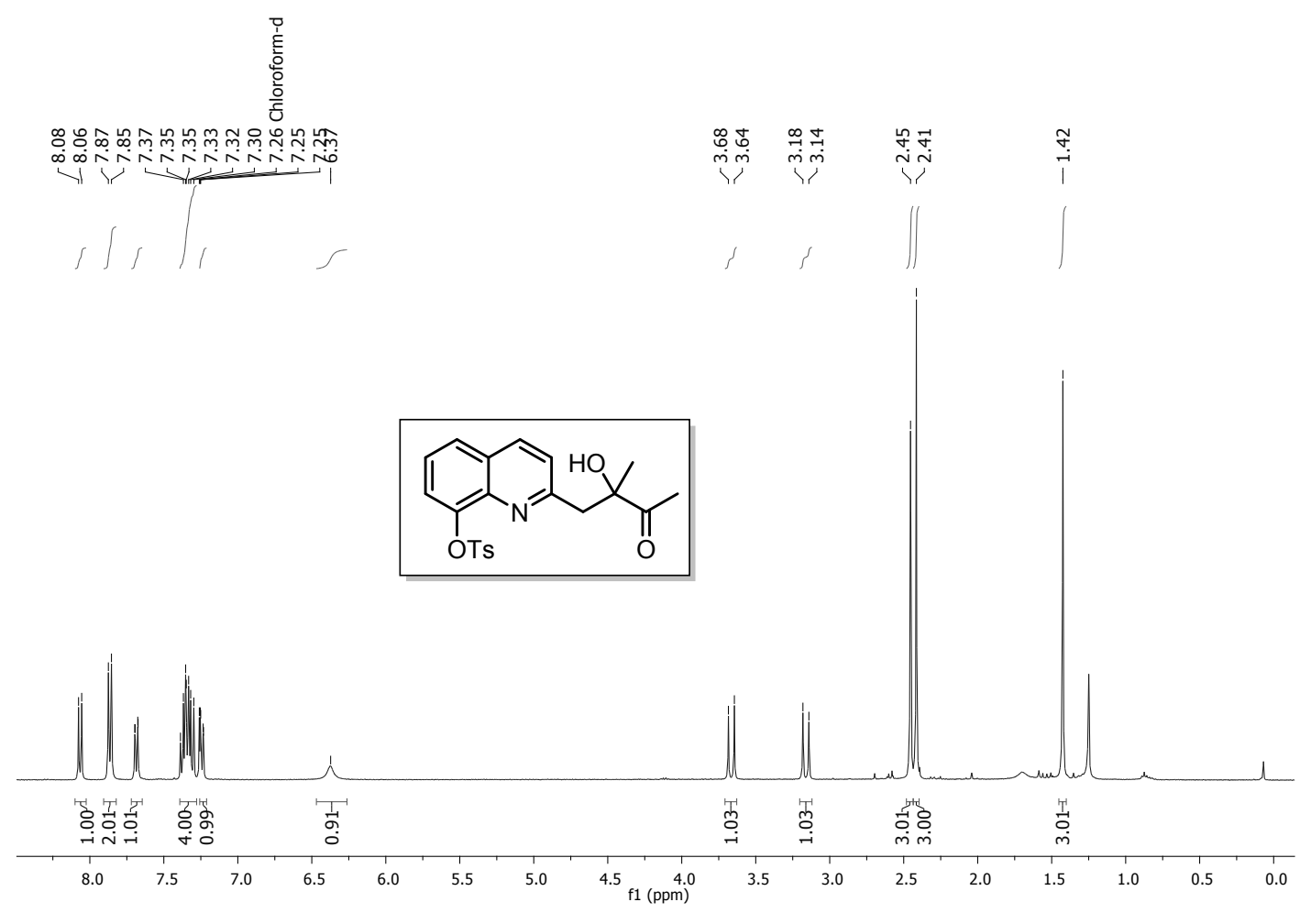

Figure S 36: ${ }^{1} \mathrm{H} \mathrm{NMR}\left(400 \mathrm{MHz}, \mathrm{CDCl}_{3}\right.$ ) of the compound 2-(2-hydroxy-2-methyl-3oxobutyl)quinolin-8-yl 4-methylbenzenesulfonate (3ja) in $\mathrm{CDCl}_{3}$

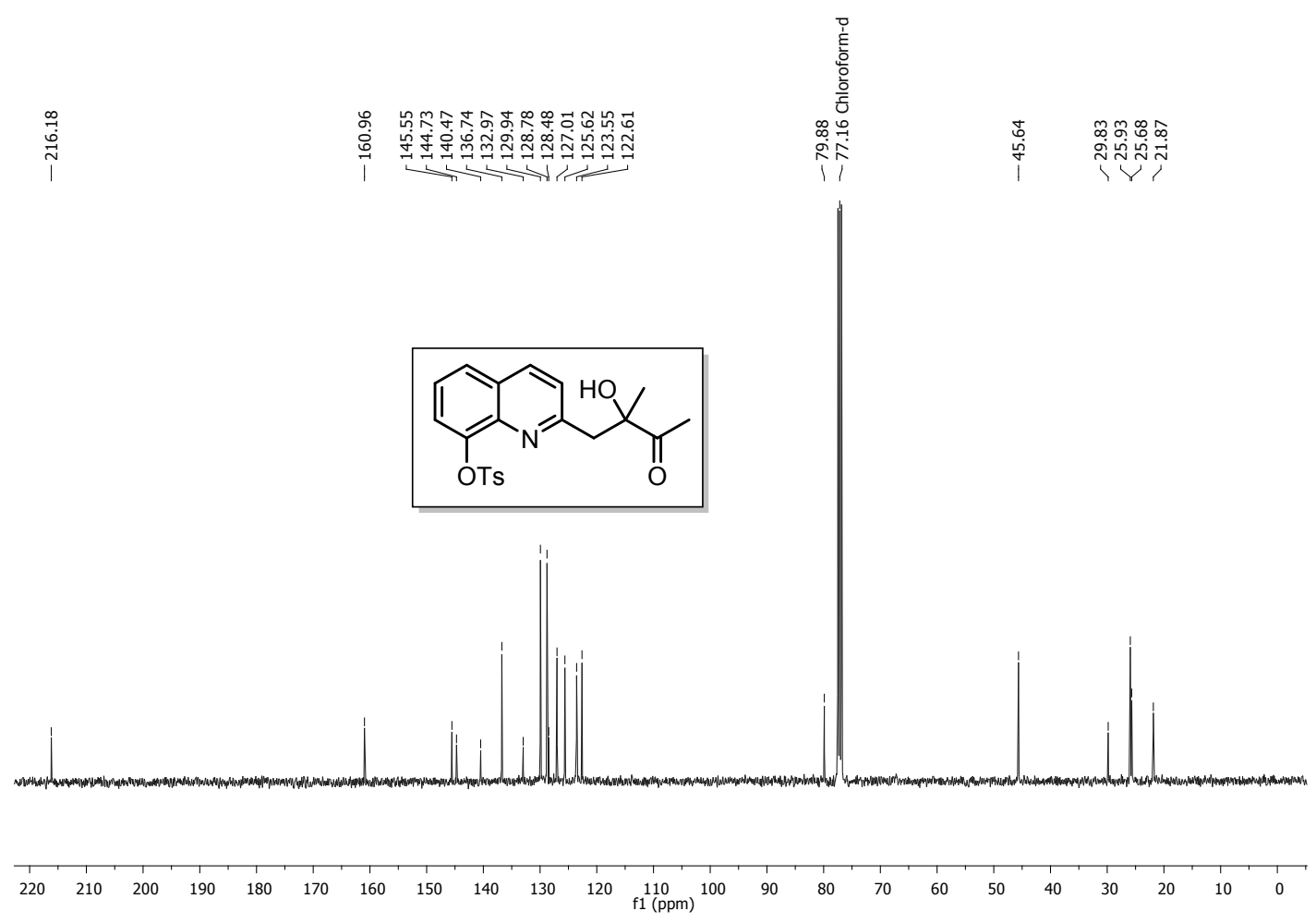

Figure S 37: ${ }^{13} \mathrm{C}$ NMR (101 MHz, $\mathrm{CDCl}_{3}$ ) of the compound 2-(2-hydroxy-2-methyl-3$10(\mathbf{3 j a})$ in $\mathrm{CDCl}_{3}$ 


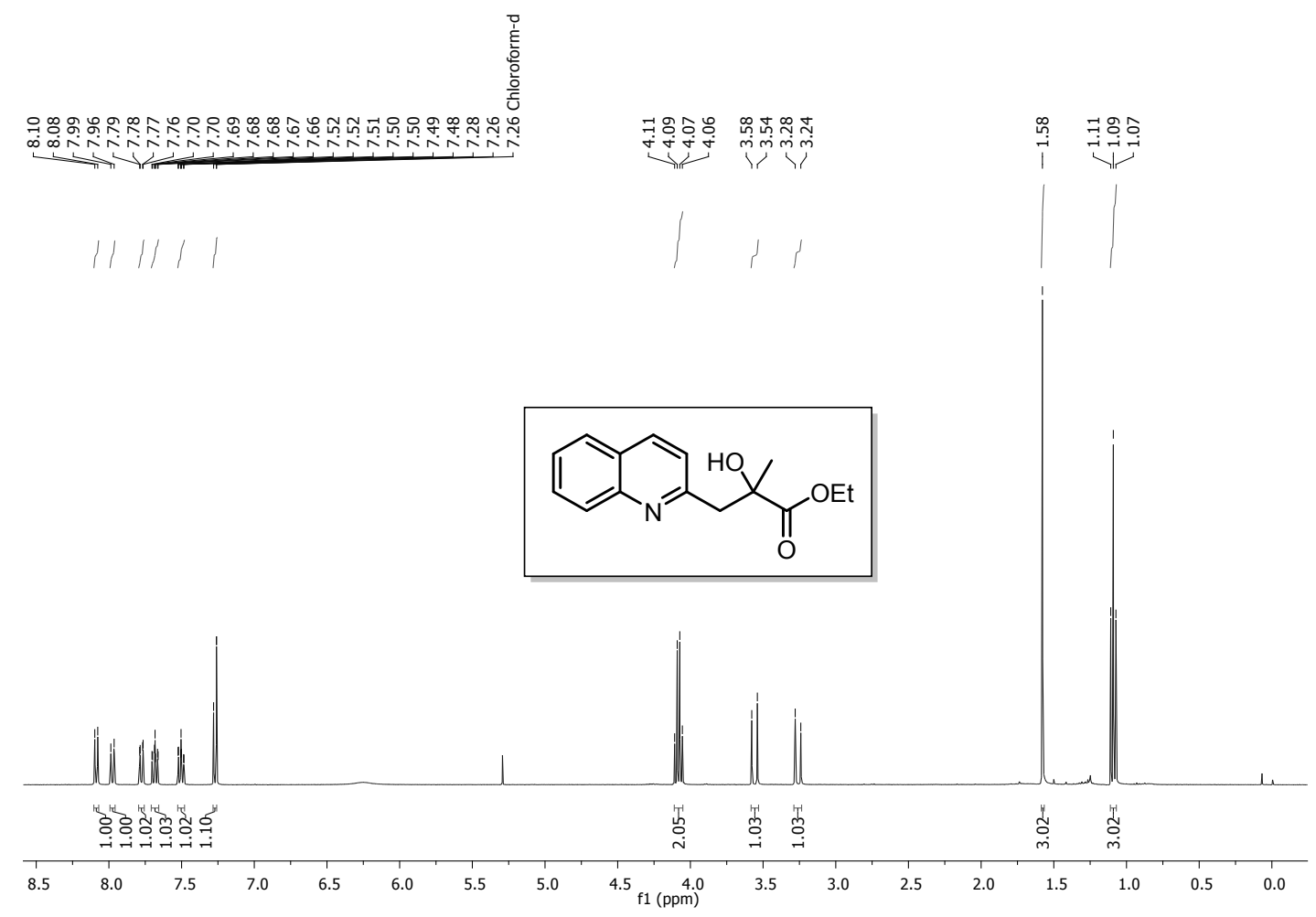

Figure S 38: ${ }^{1} \mathrm{H}$ NMR (400 MHz, $\mathrm{CDCl}_{3}$ ) of compound ethyl 2-hydroxy-2-methyl-3-(quinolin2-yl)propanoate (3ab) in $\mathrm{CDCl}$

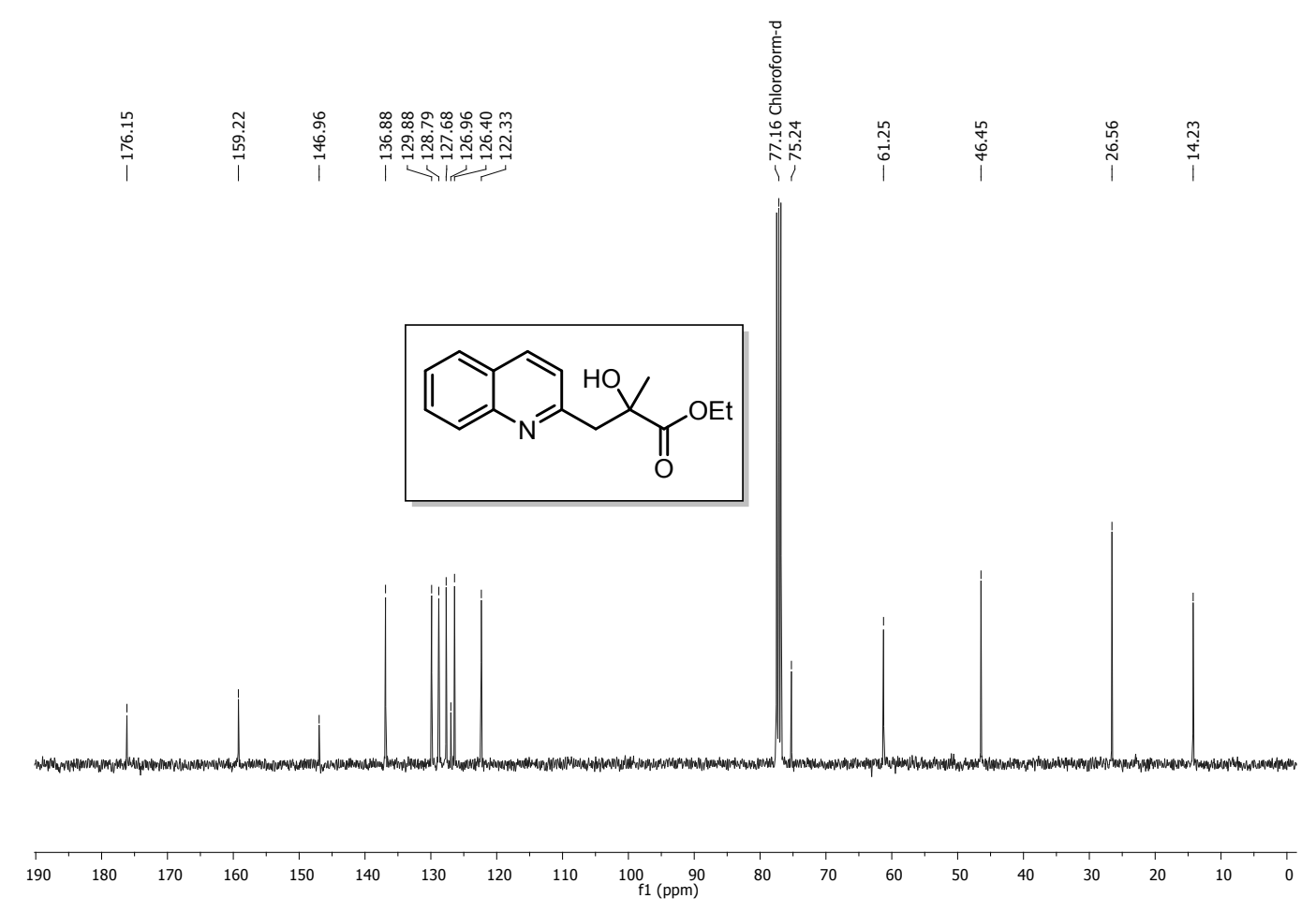

Figure S 39: ${ }^{13} \mathrm{C}$ NMR $\left(101 \mathrm{MHz}, \mathrm{CDCl}_{3}\right.$ ) of compound ethyl 2-hydroxy-2-methyl-3-(quinolin2-yl)propanoate (3ab) in $\mathrm{CDCl}_{3}$ 


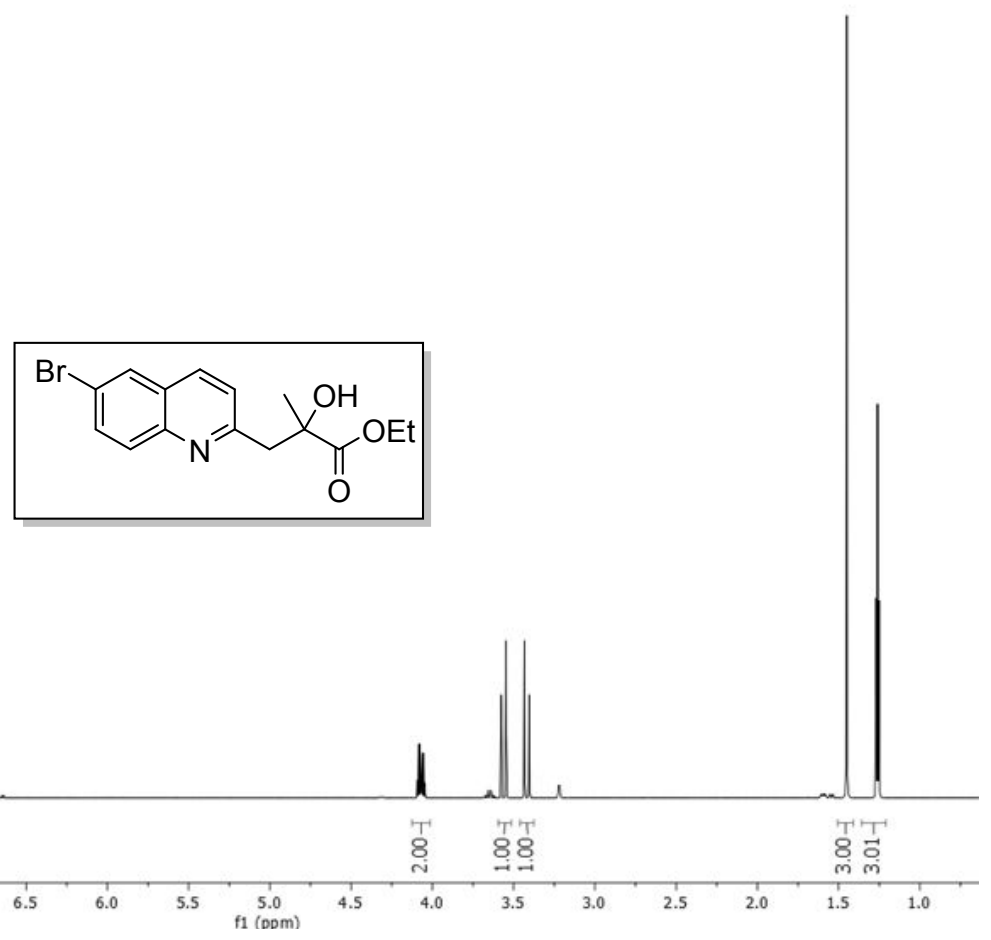

Figure S 40: ${ }^{1} \mathrm{H}$ NMR (400 MHz, $\mathrm{CDCl}_{3}$ ) of compound ethyl 3-(6-bromoquinolin-2-yl)-2hydroxy-2-methylpropanoate (3bb) in $\mathrm{CDCl}_{3}$

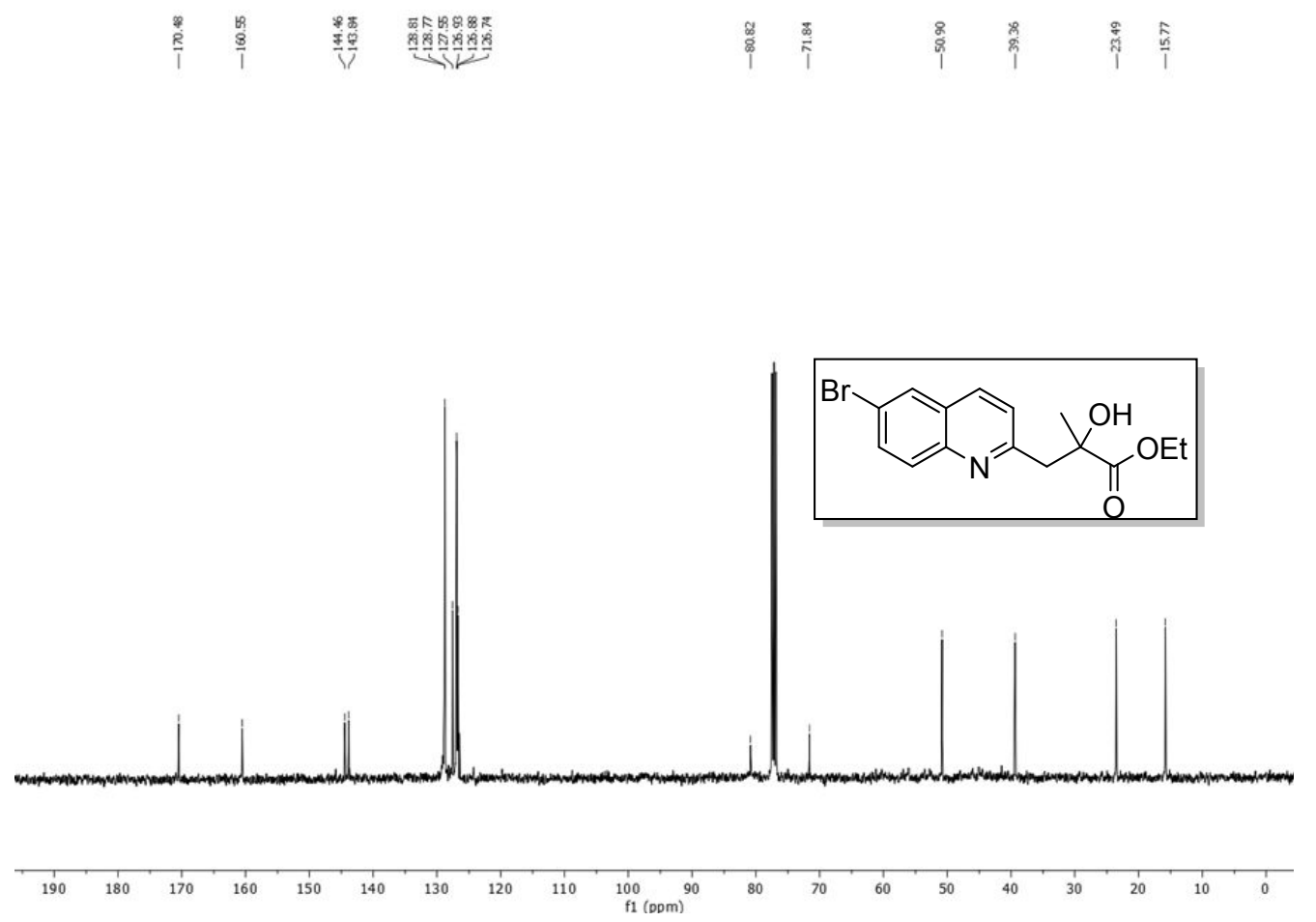

Figure S 41: ${ }^{13} \mathrm{C}$ NMR (101 MHz, $\mathrm{CDCl}_{3}$ ) of compound ethyl 3-(6-bromoquinolin-2-yl)-2hydroxy-2-methylpropanoate (3bb) in $\mathrm{CDCl}_{3}$ 


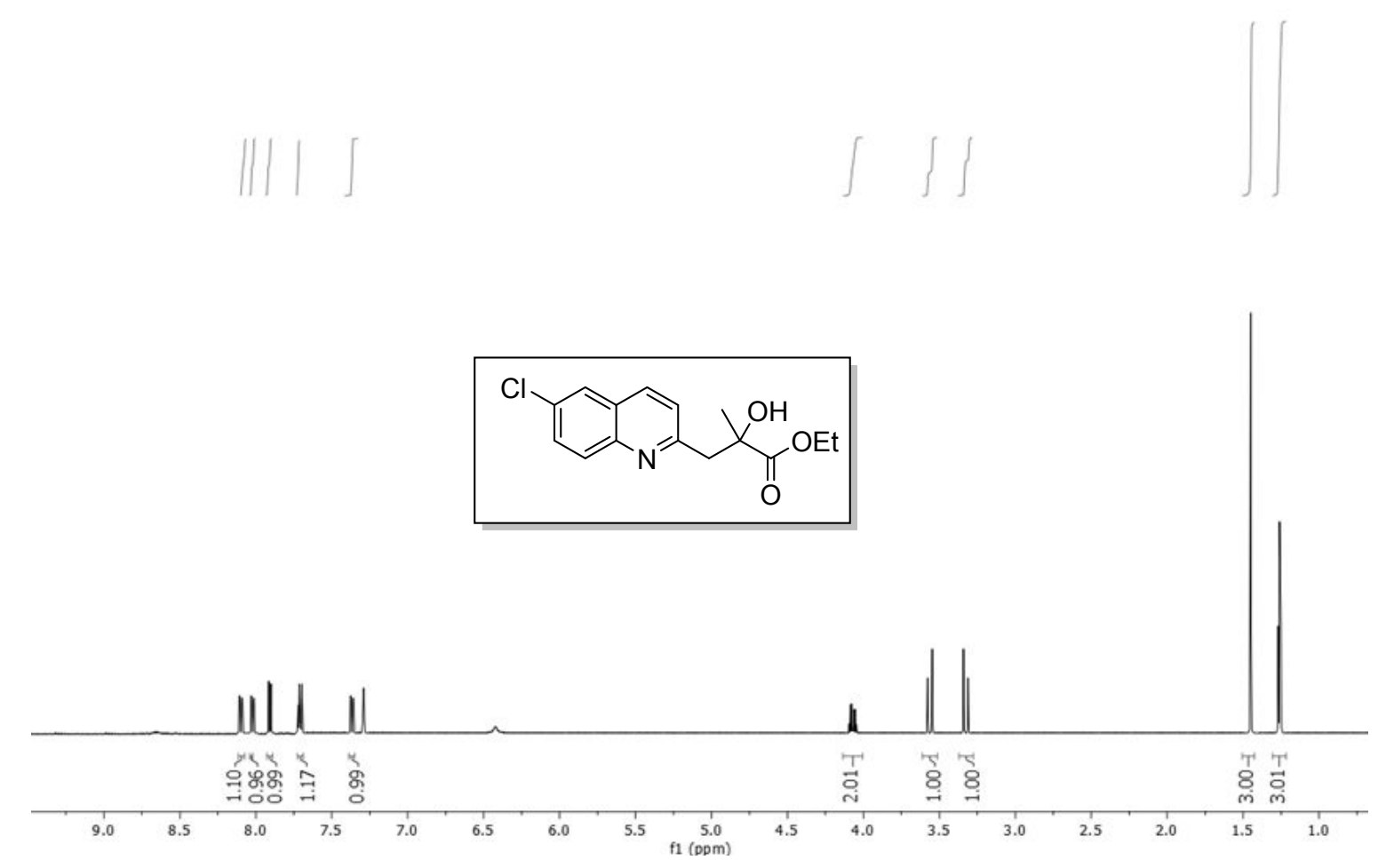

Figure S 42: ${ }^{1} \mathrm{H}$ NMR (400 MHz, $\mathrm{CDCl}_{3}$ ) of compound ethyl 3-(6-chloroquinolin-2-yl)-2-hydroxy2-methylpropanoate (3cb) in $\mathrm{CDCl}_{3}$

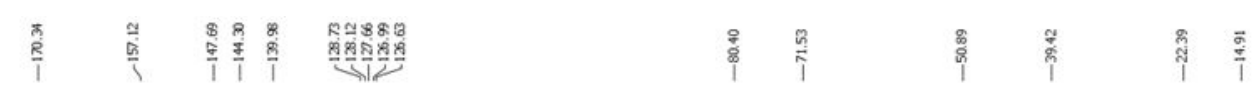
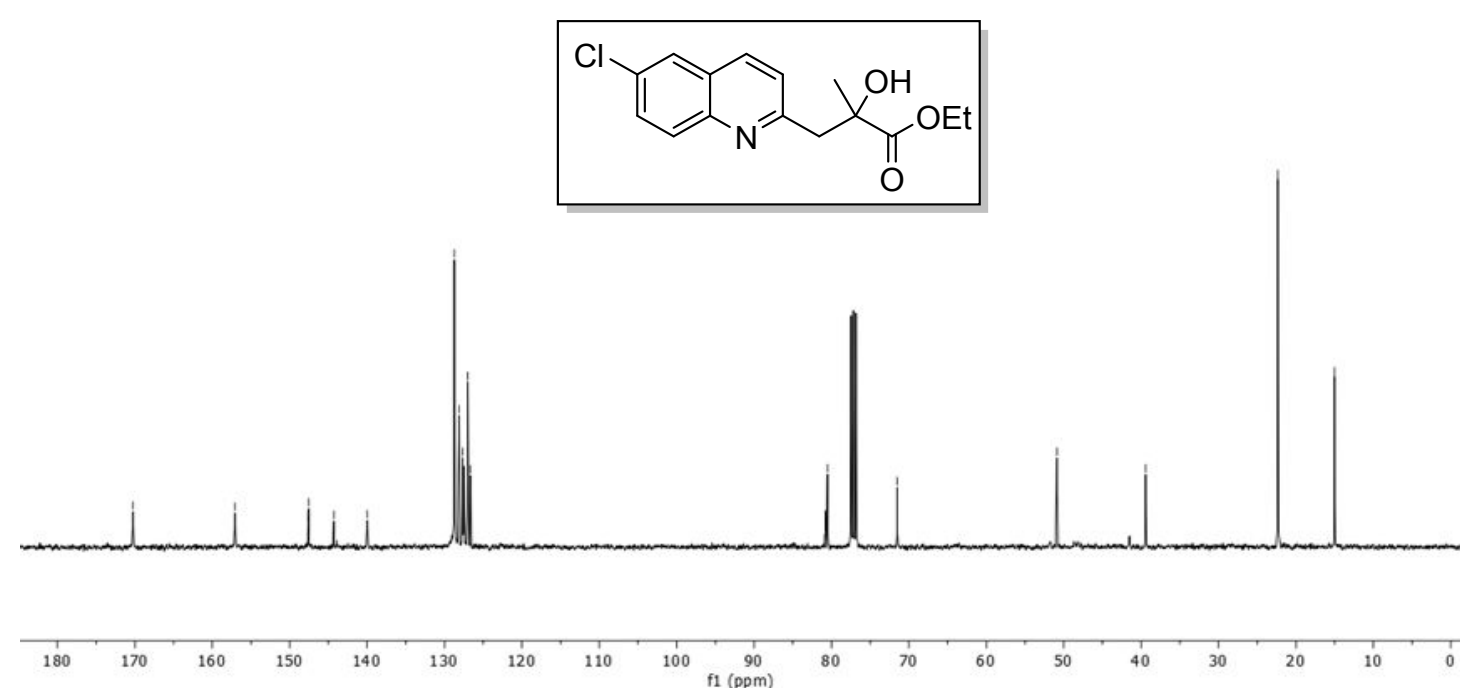

Figure S 43: ${ }^{13} \mathrm{C}$ NMR (101 MHz, $\mathrm{CDCl}_{3}$ ) of compound ethyl 3-(6-chloroquinolin-2-yl)-2hydroxy-2-methylpropanoate (3cb) in $\mathrm{CDCl}_{3}$ 

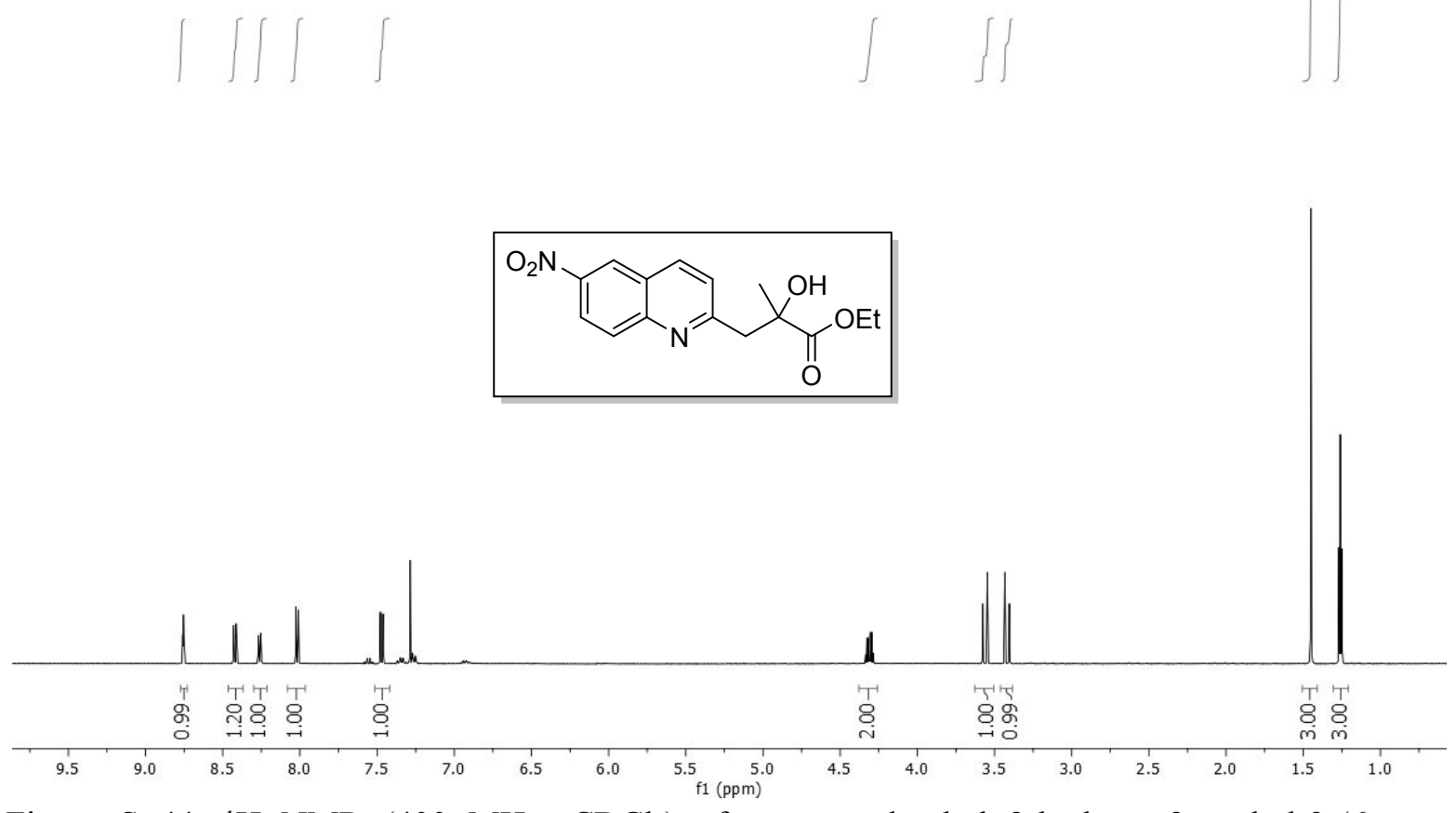

Figure S 44: ${ }^{1} \mathrm{H}$ NMR (400 $\mathrm{MHz}, \mathrm{CDCl}_{3}$ ) of compound ethyl 2-hydroxy-2-methyl-3-(6nitroquinolin-2-yl)propanoate (3db) in $\mathrm{CDCl}_{3}$
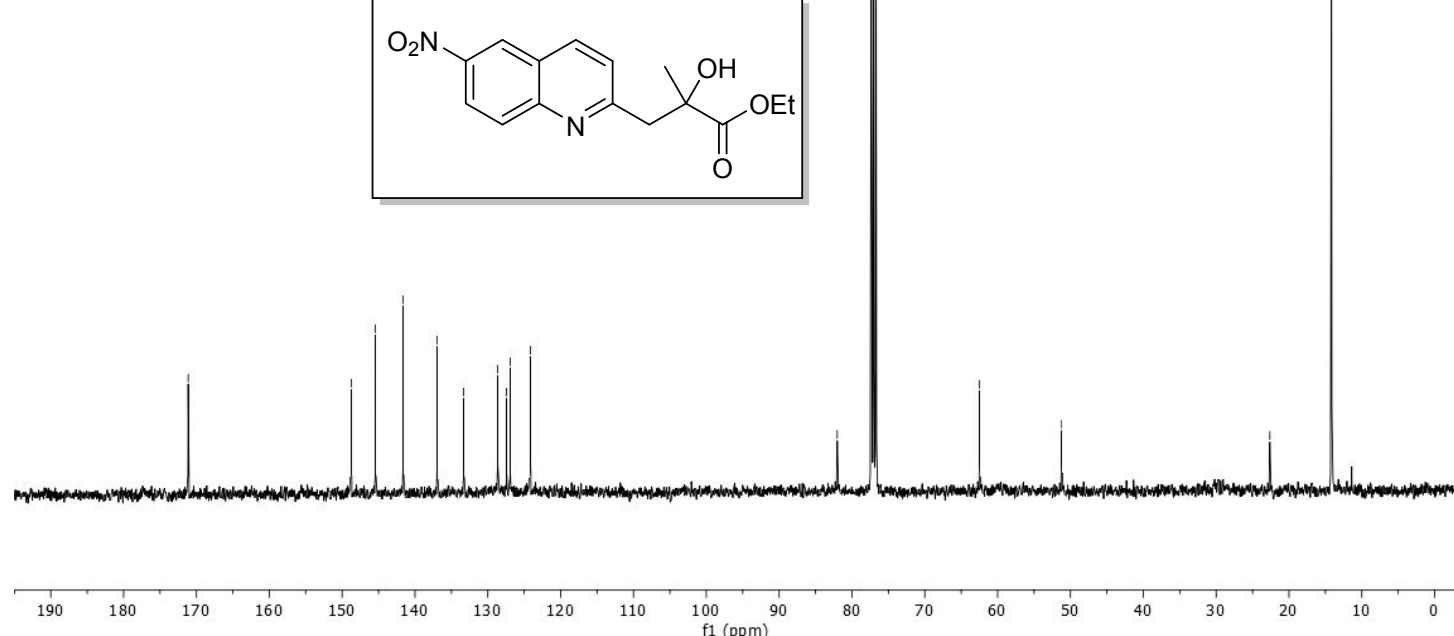

Figure $\mathrm{S} 45:{ }^{13} \mathrm{C}$ NMR (101 MHz, $\mathrm{CDCl}_{3}$ ) of compound ethyl 2-hydroxy-2-methyl-3-(6nitroquinolin-2-yl)propanoate (3db) in $\mathrm{CDCl}_{3}$ 


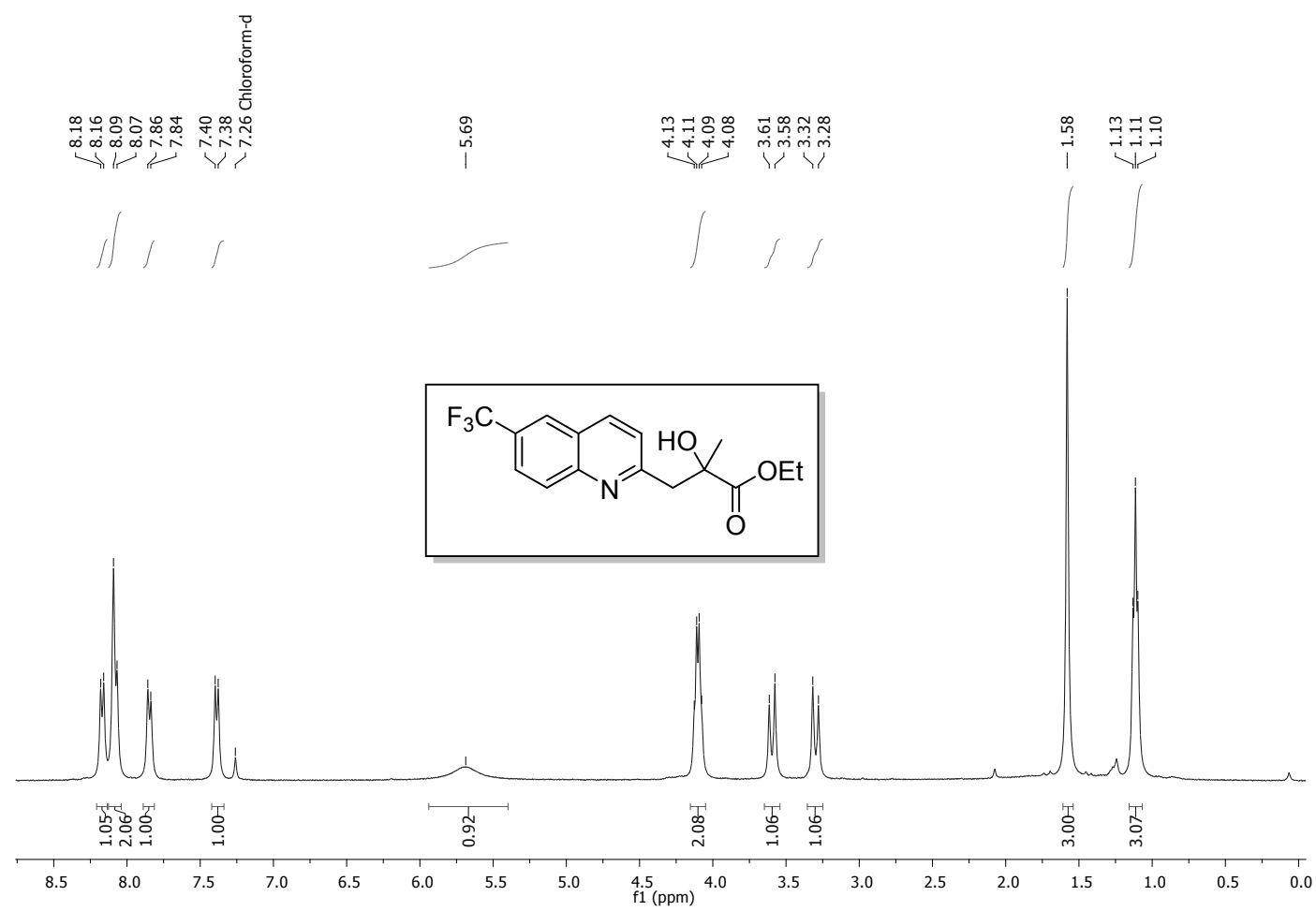

Figure S 46: ${ }^{1} \mathrm{H}$ NMR (400 MHz, $\mathrm{CDCl}_{3}$ ) of compound ethyl 2-hydroxy-2-methyl-3-(6(trifluoromethyl)quinolin-2-yl)propanoate (3eb) in $\mathrm{CDCl}_{3}$
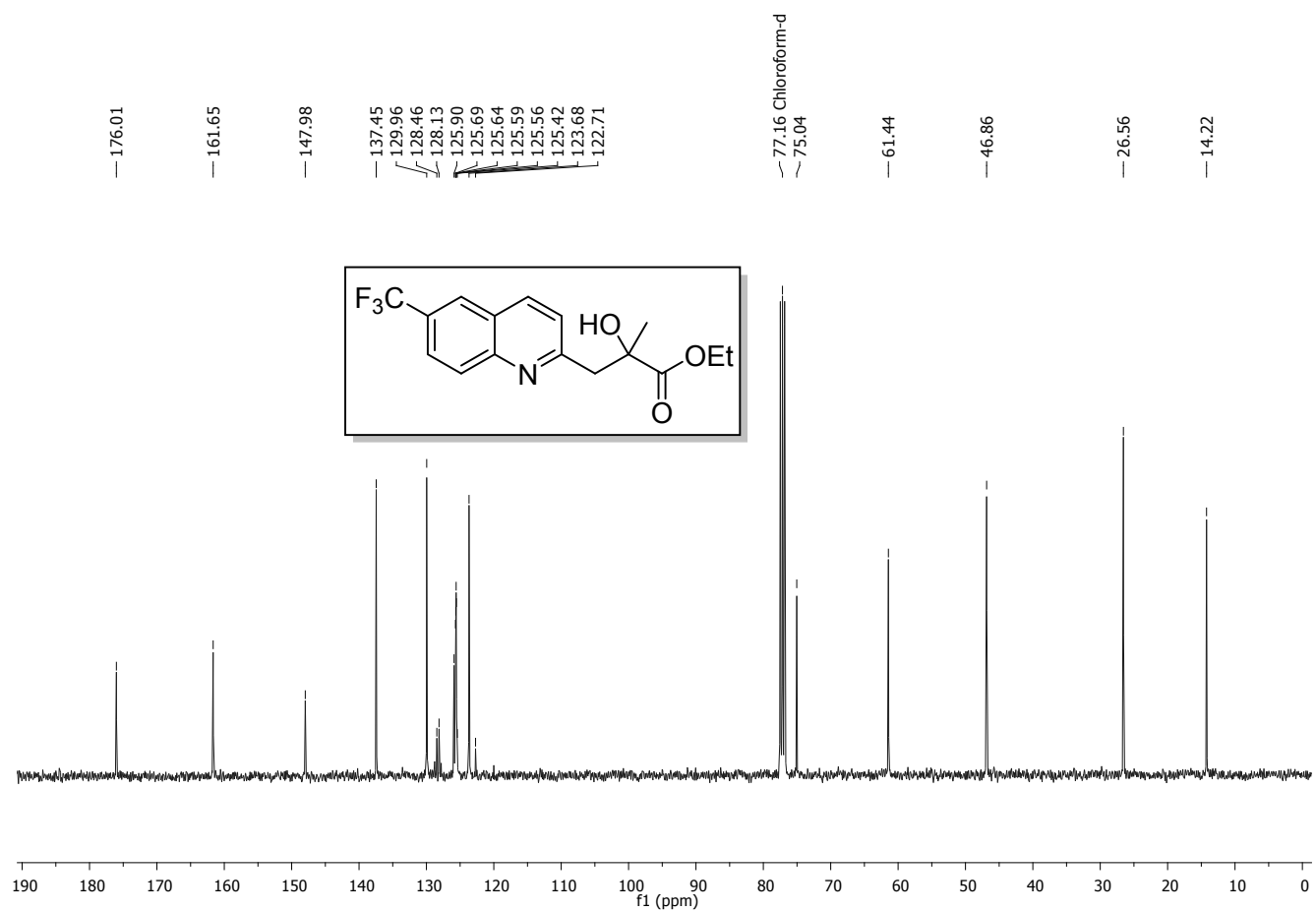

Figure S 47: ${ }^{13} \mathrm{C}$ NMR (101 MHz, $\mathrm{CDCl}_{3}$ ) of compound ethyl 2-hydroxy-2-methyl-3-(6(trifluoromethyl)quinolin-2-yl)propanoate (3eb) in $\mathrm{CDCl}_{3}$. 


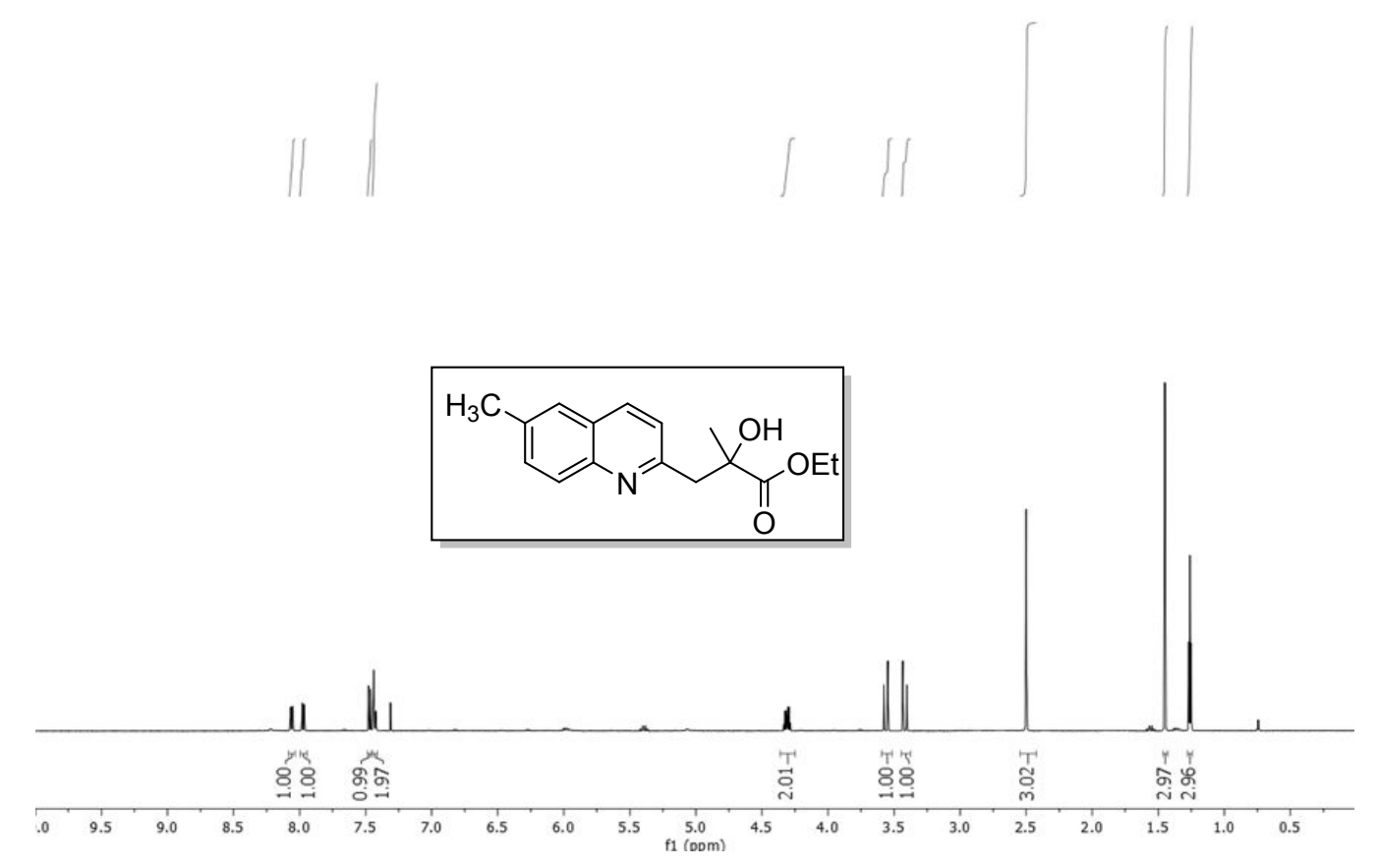

Figure S 48: ${ }^{1} \mathrm{H}$ NMR (400 MHz, $\left.\mathrm{CDCl}_{3}\right)$ of compound ethyl 2-hydroxy-2-methyl-3-(6methylquinolin-2-yl)propanoate (3gb) in $\mathrm{CDCl}_{3}$

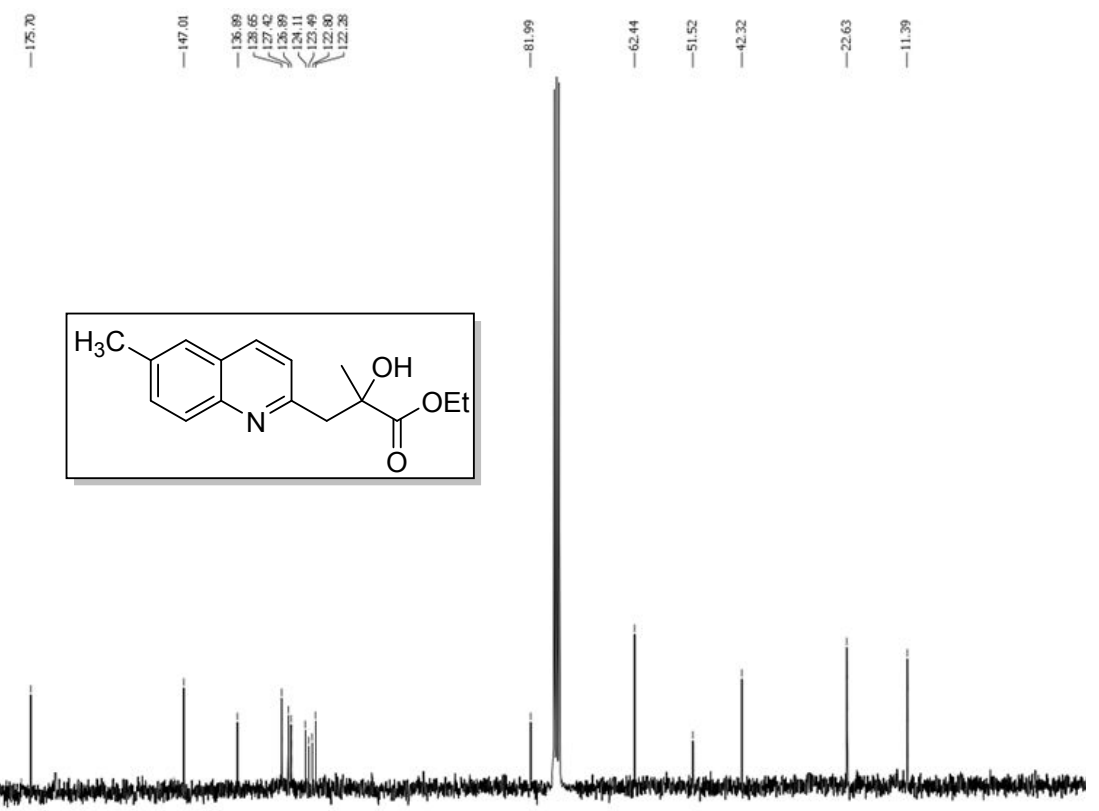

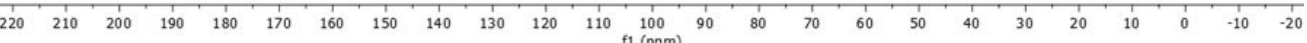

Figure S 49: ${ }^{13} \mathrm{C}$ NMR (101 MHz, $\mathrm{CDCl}_{3}$ ) of compound ethyl 2-hydroxy-2-methyl-3-(6methylquinolin-2-yl)propanoate $(\mathbf{3 g b})$ in $\mathrm{CDCl}_{3}$ in $\mathrm{CDCl}_{3}$ 


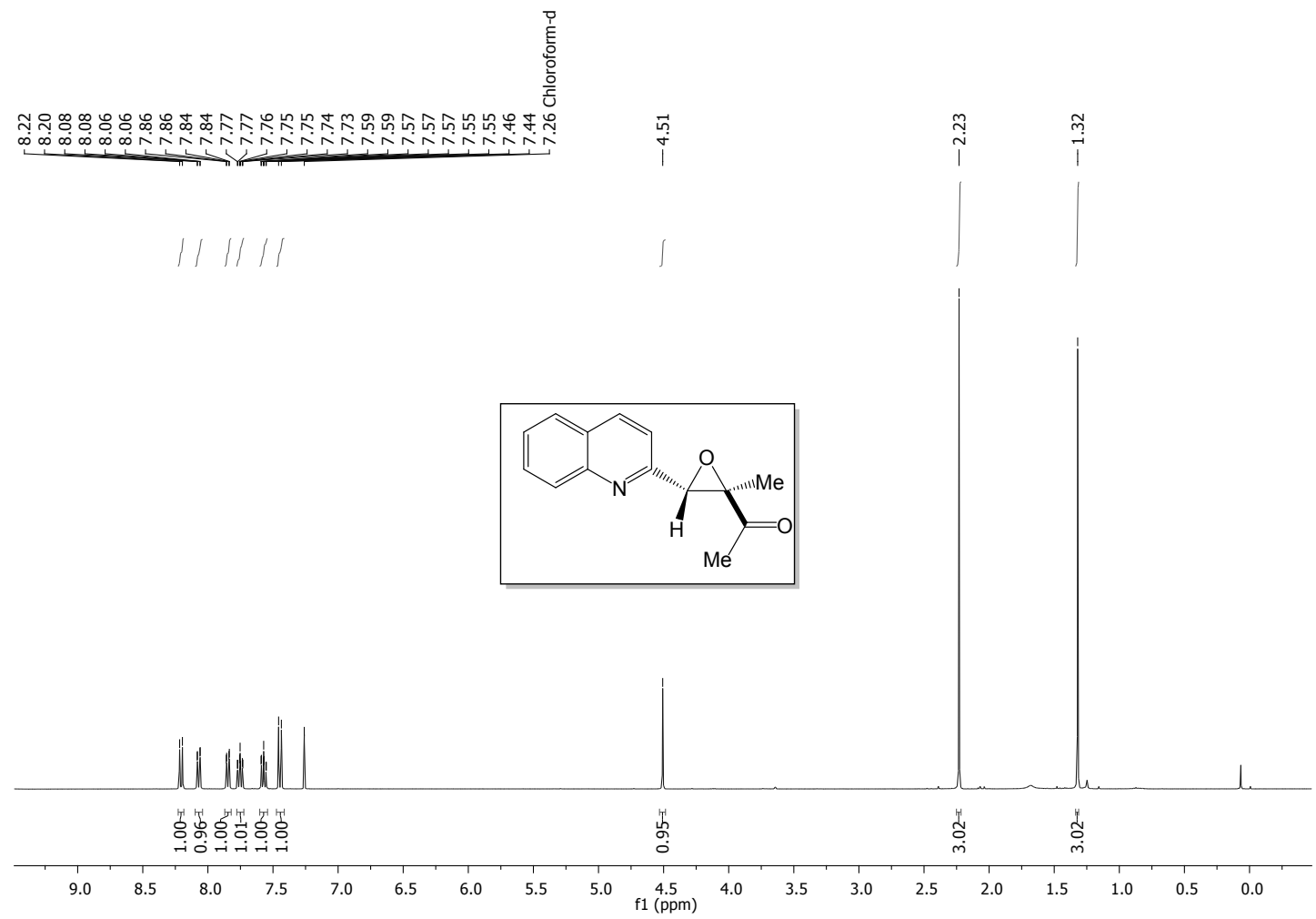

Figure S 50: ${ }^{1} \mathrm{H}$ NMR (400 MHz, $\mathrm{CDCl}_{3}$ ) of compound 1-(2-methyl-3-(quinolin-2-yl)oxiran-2yl)ethan-1-one (5) in $\mathrm{CDCl}_{3}$

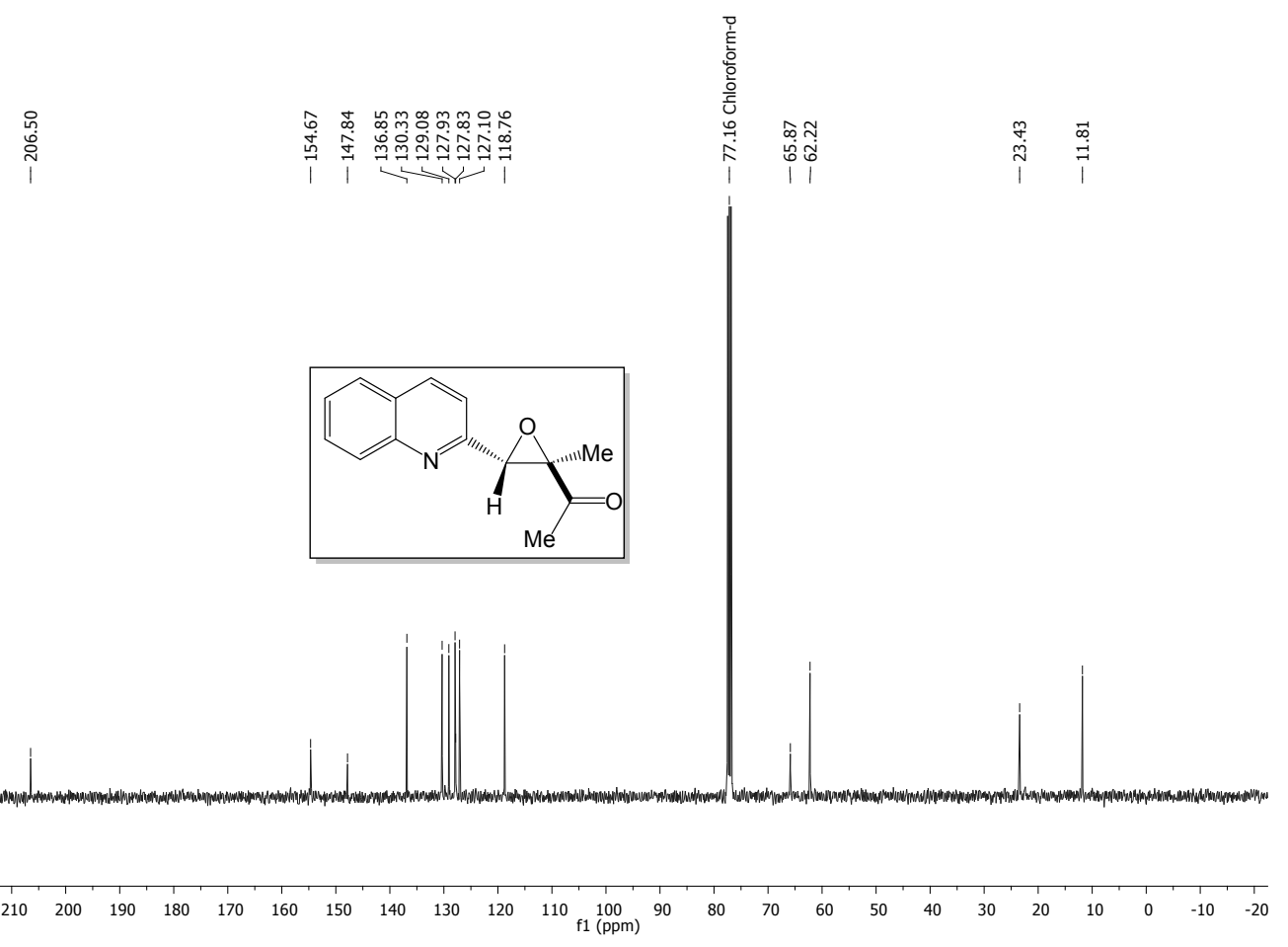

Figure S 51: ${ }^{13} \mathrm{C}$ NMR (101 MHz, $\mathrm{CDCl}_{3}$ ) of compound 1-(2-methyl-3-(quinolin-2-yl)oxiran-2yl)ethan-1-one (5) in $\mathrm{CDCl}_{3}$ 

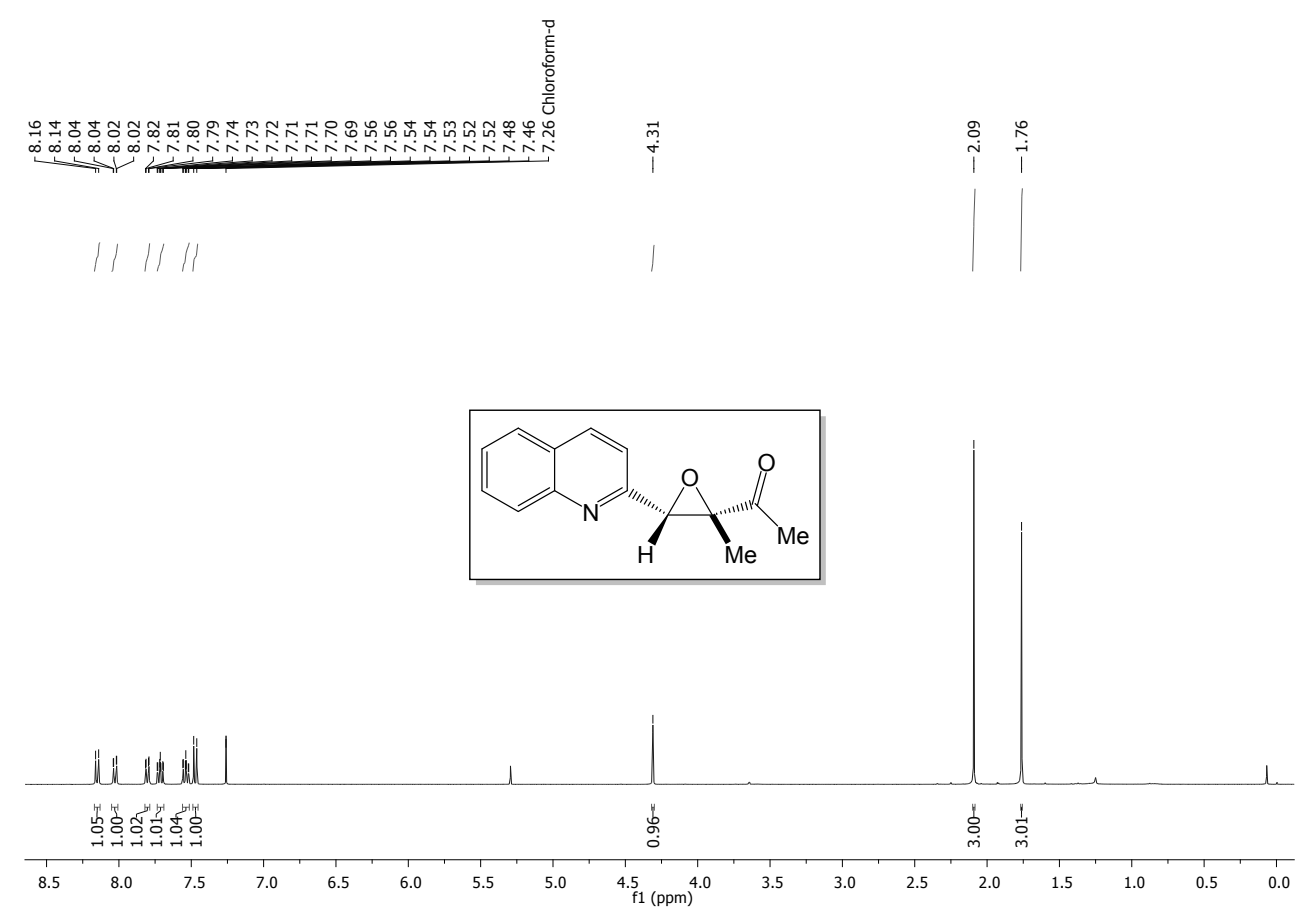

Figure S 52: ${ }^{1} \mathrm{H}$ NMR (400 MHz, $\mathrm{CDCl}_{3}$ ) of compound 1-(2-methyl-3-(quinolin-2-yl)oxiran-2yl)ethan-1-one (5') in $\mathrm{CDCl}_{3}$
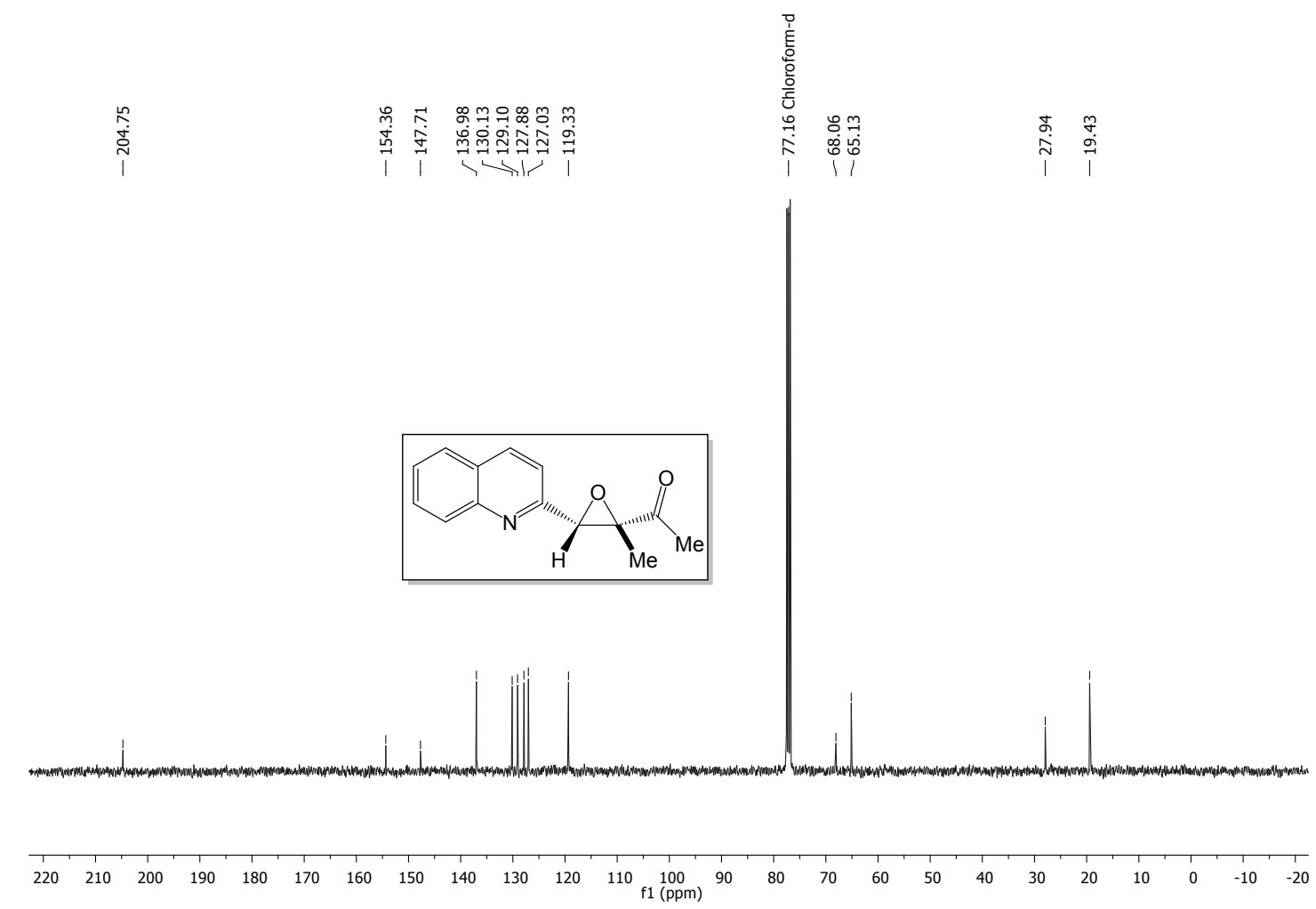

11

Figure S 53: ${ }^{13} \mathrm{C}$ NMR (101 MHz, $\mathrm{CDCl}_{3}$ ) of compound 1-(2-methyl-3-(quinolin-2-yl)oxiran-2yl)ethan-1-one (5') in $\mathrm{CDCl}_{3}$ 


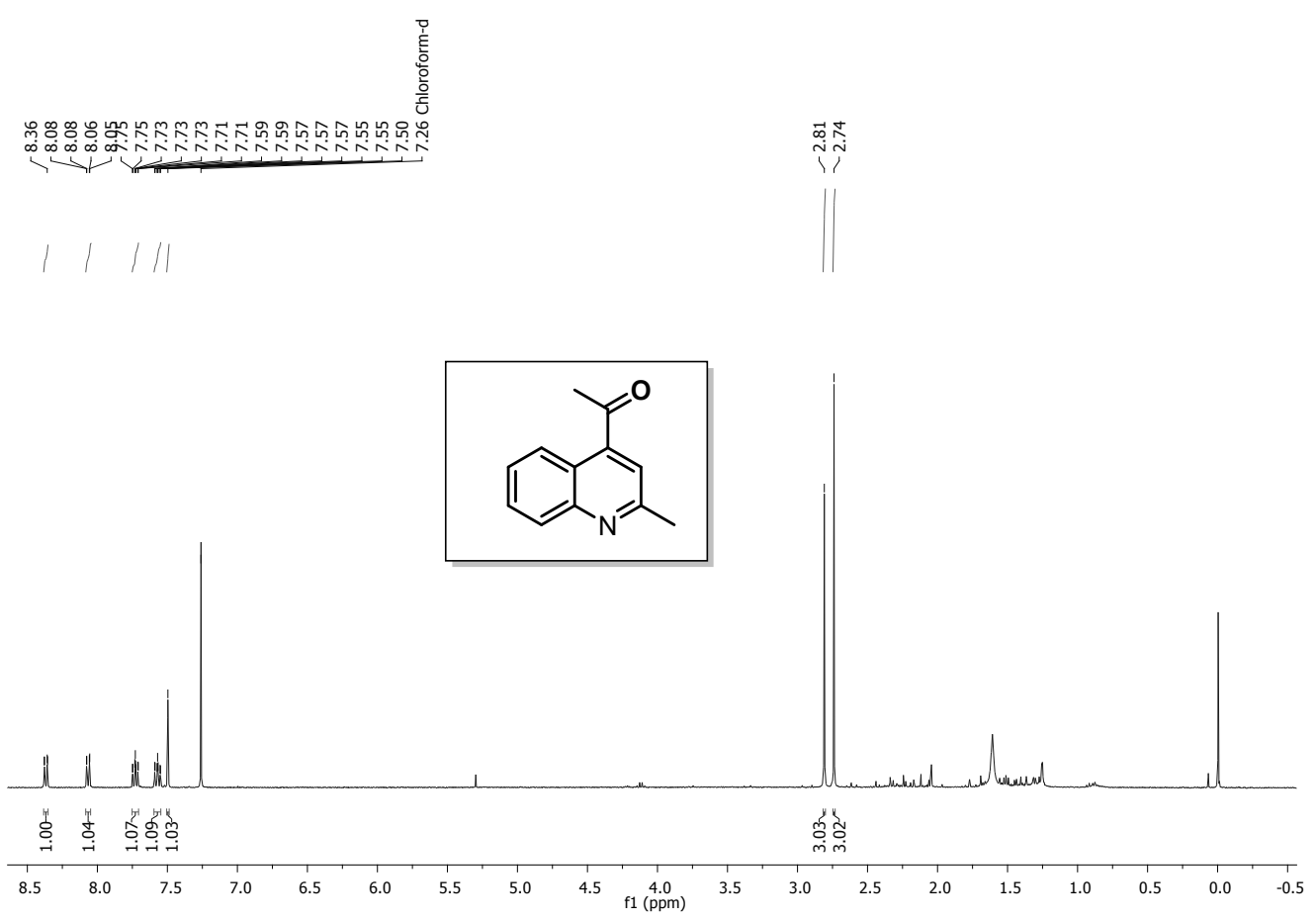

Figure S 54: ${ }^{1} \mathrm{H} \mathrm{NMR} \mathrm{(400} \mathrm{MHz,} \mathrm{CDCl}_{3}$ ) of compound 1-(2-methylquinolin-4-yl)ethan-1-one (4) ${ }^{4}$ in $\mathrm{CDCl}_{3}$
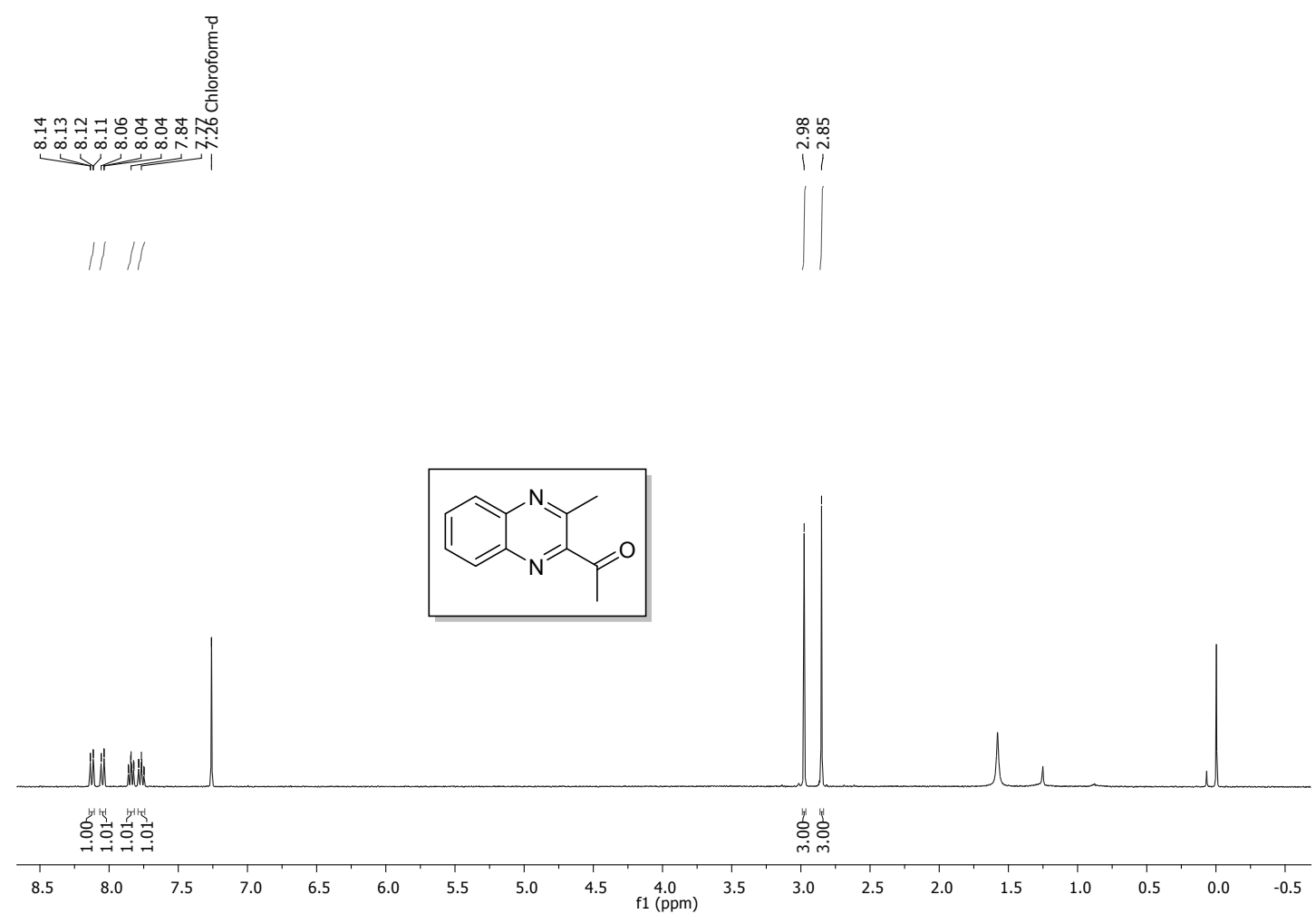

Figure S 55: ${ }^{1} \mathrm{H}$ NMR (400 MHz, $\mathrm{CDCl}_{3}$ ) of compound 1-(3-methylquinoxalin-2-yl)ethan-1one $(7 \mathbf{a})^{5}$ in $\mathrm{CDCl}_{3}$ 

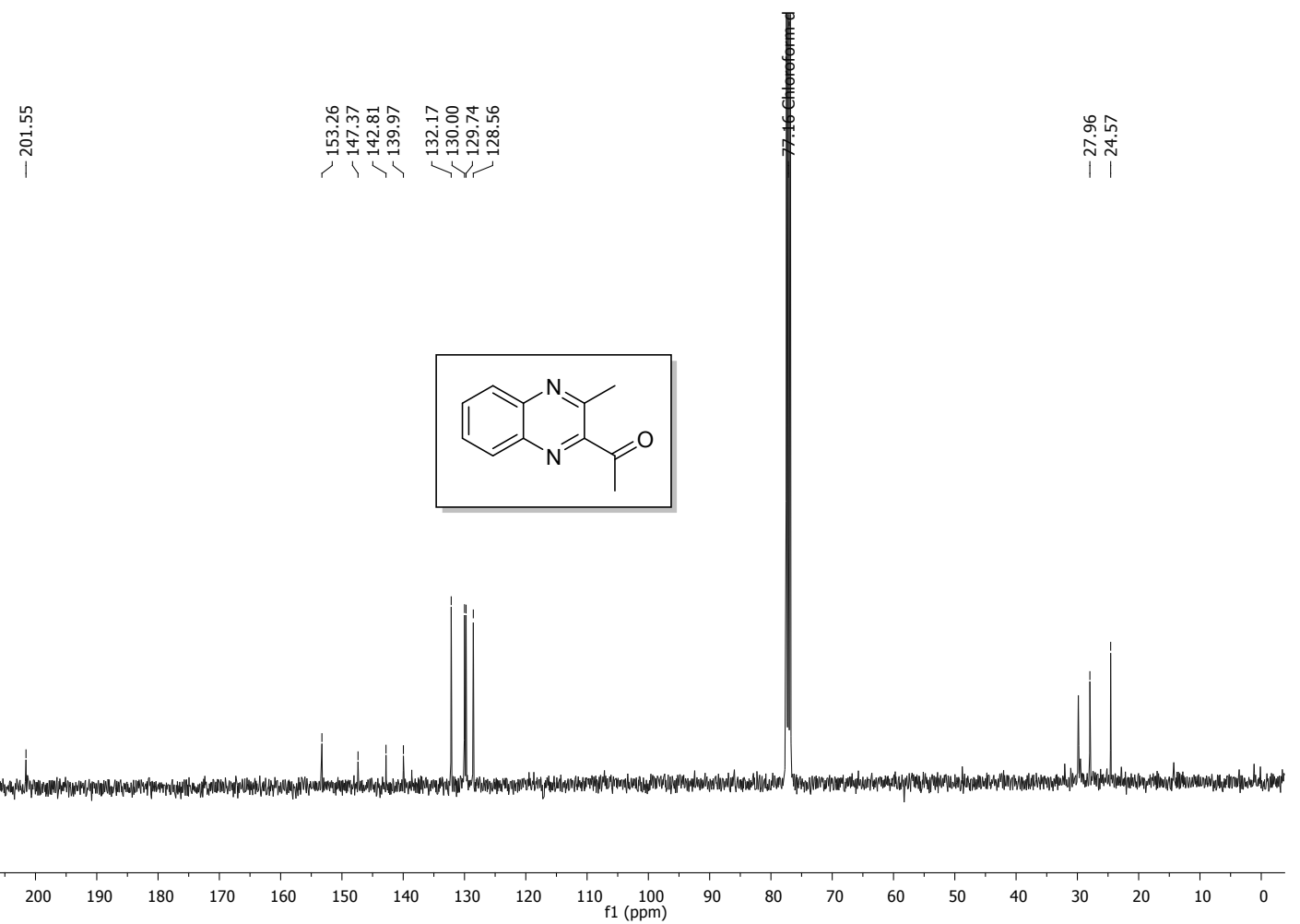

Figure S 56: ${ }^{13} \mathrm{C}$ NMR (101 MHz, $\mathrm{CDCl}_{3}$ ) of compound 1-(3-methylquinoxalin-2-yl)ethan-1one $(7 \mathbf{a})^{5}$ in $\mathrm{CDCl}_{3}$
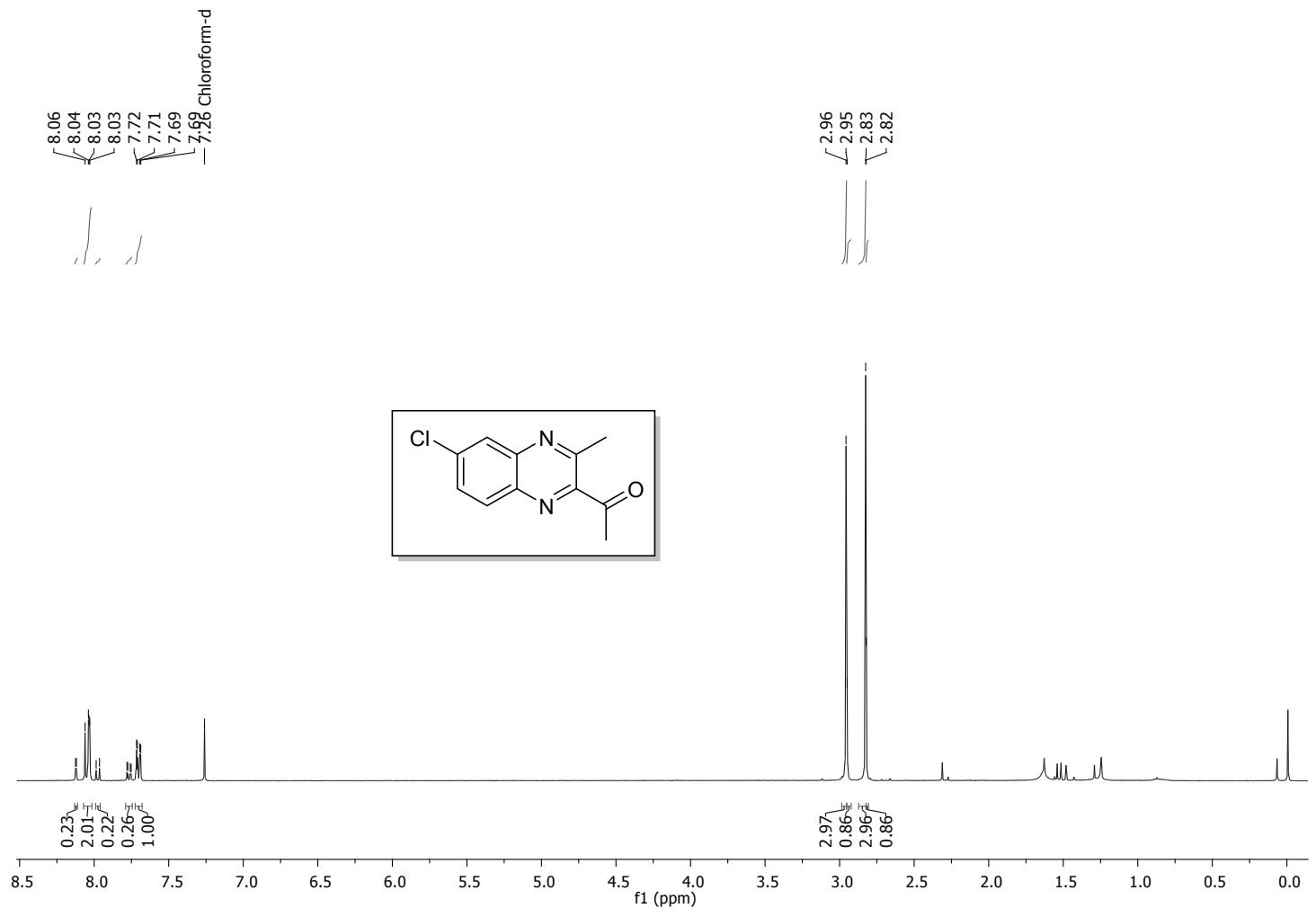

Figure S 57: ${ }^{1} \mathrm{H} \mathrm{NMR}\left(400 \mathrm{MHz}, \mathrm{CDCl}_{3}\right)$ of compound 1-(6-chloro-3-methylquinoxalin-2yl)ethan-1-one (7b) $)^{5}$ in $\mathrm{CDCl}_{3}$ 


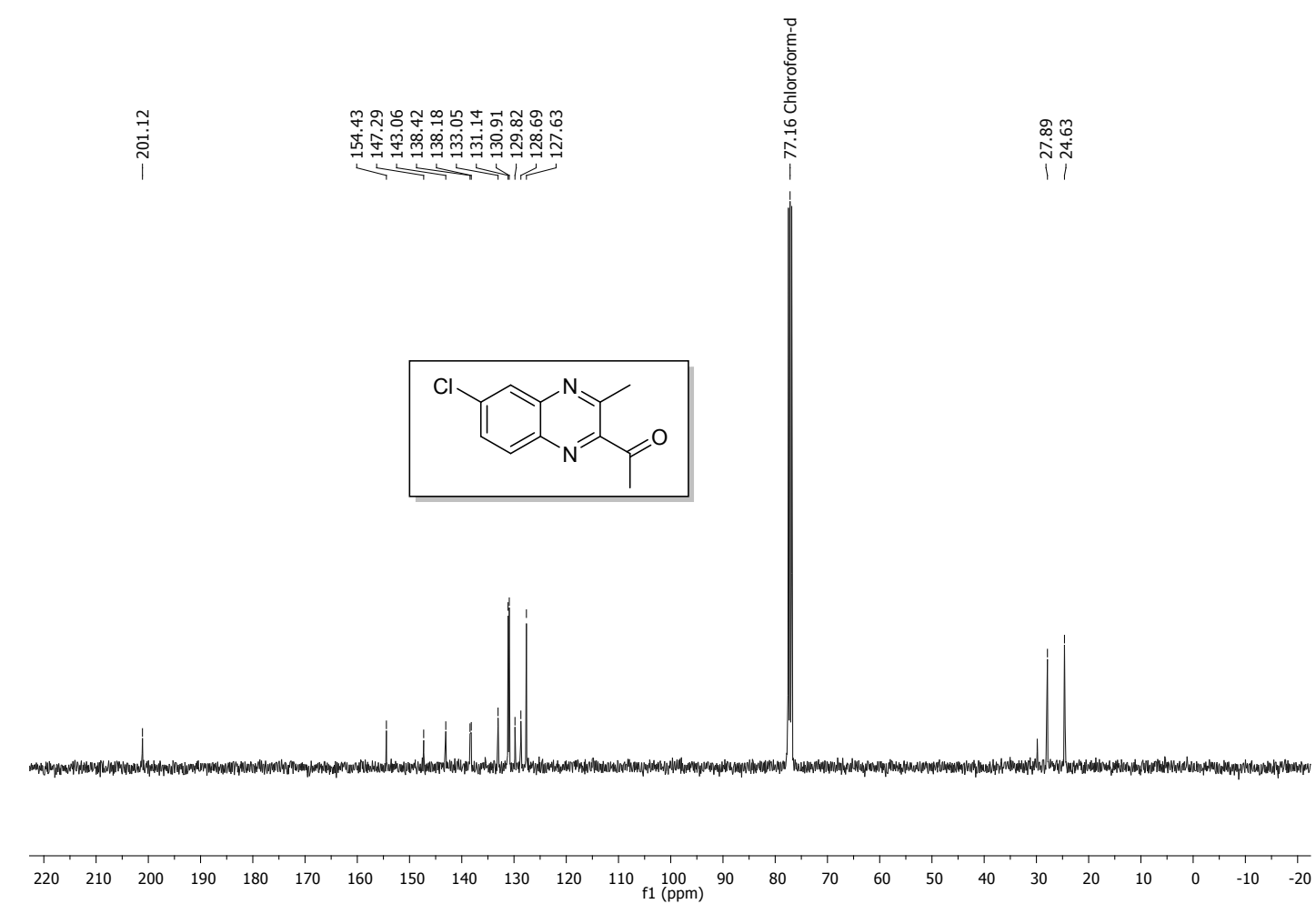

Figure S 58: ${ }^{13} \mathrm{C}$ NMR (101 MHz, $\mathrm{CDCl}_{3}$ ) of compound 1-(6-chloro-3-methylquinoxalin-2yl)ethan-1-one (7b) ${ }^{2}$ in $\mathrm{CDCl}_{3}$.

\section{DEPT 135, HSQC, IR DATA}
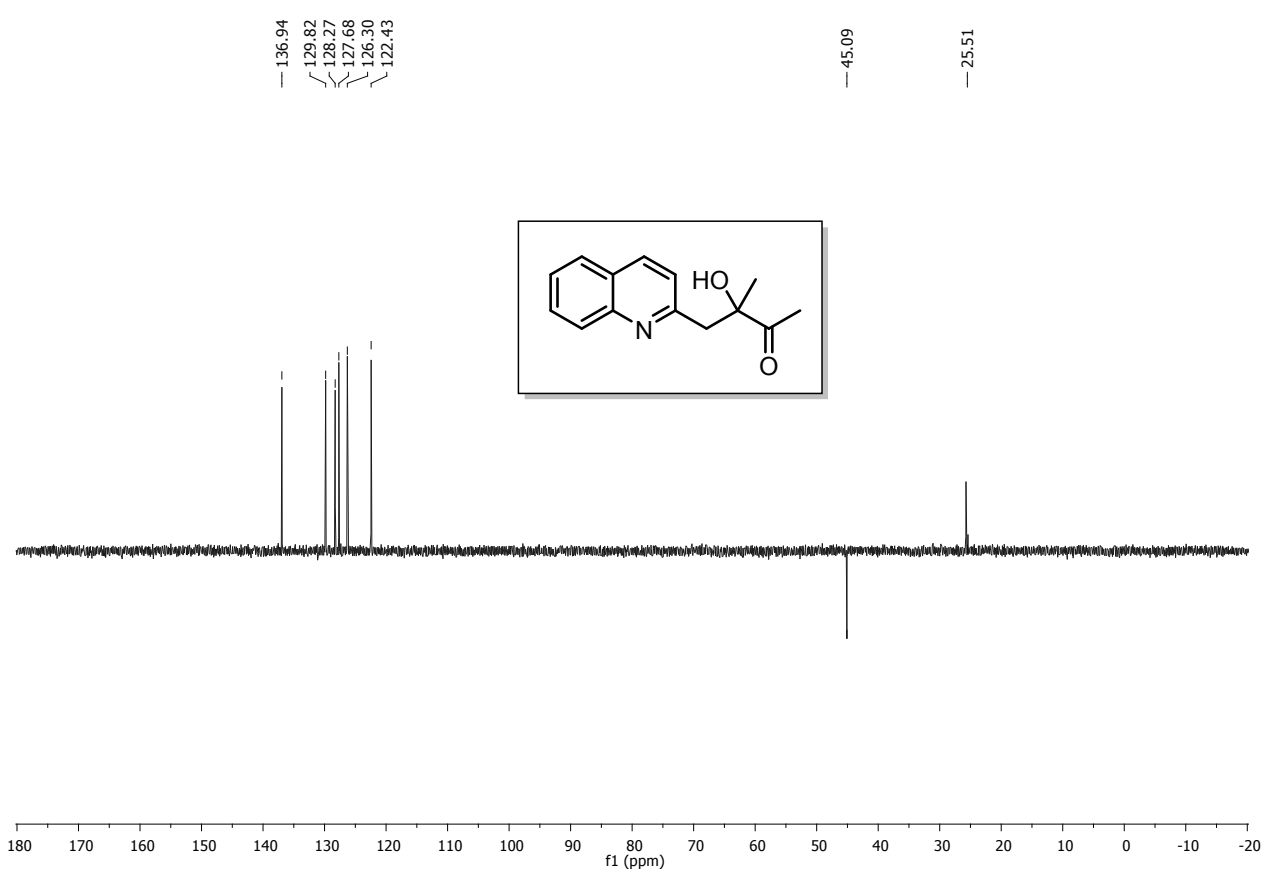

Figure S 59: DEPT 135-1K (101 MHz, $\mathrm{CDCl}_{3}$ ) of compound 3-hydroxy-3-methyl-4-(quinolin2-yl)butan-2-one (3aa) 


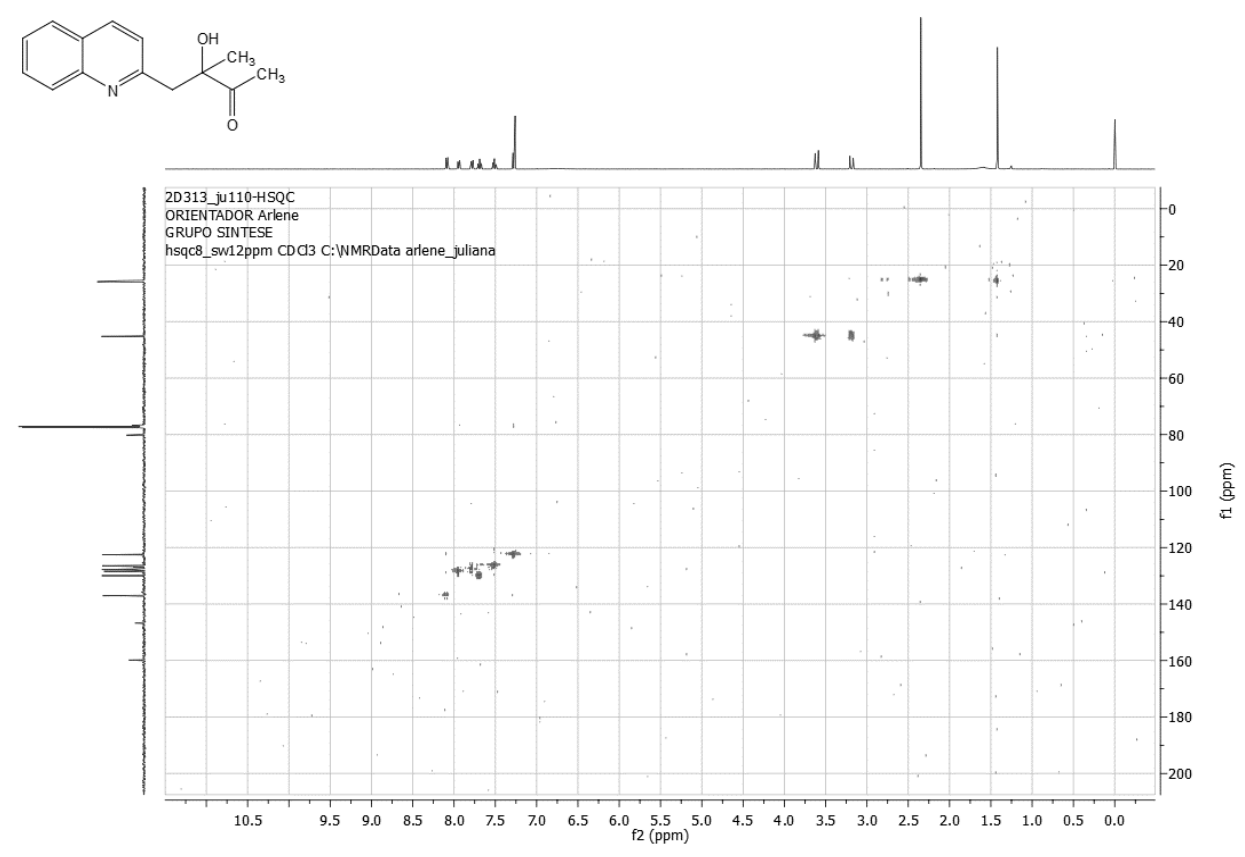

Figure S 60: HSQC $\left(\mathrm{CDCl}_{3}\right)$ of compound 3-hydroxy-3-methyl-4-(quinolin-2-yl)butan-2-one (3aa)

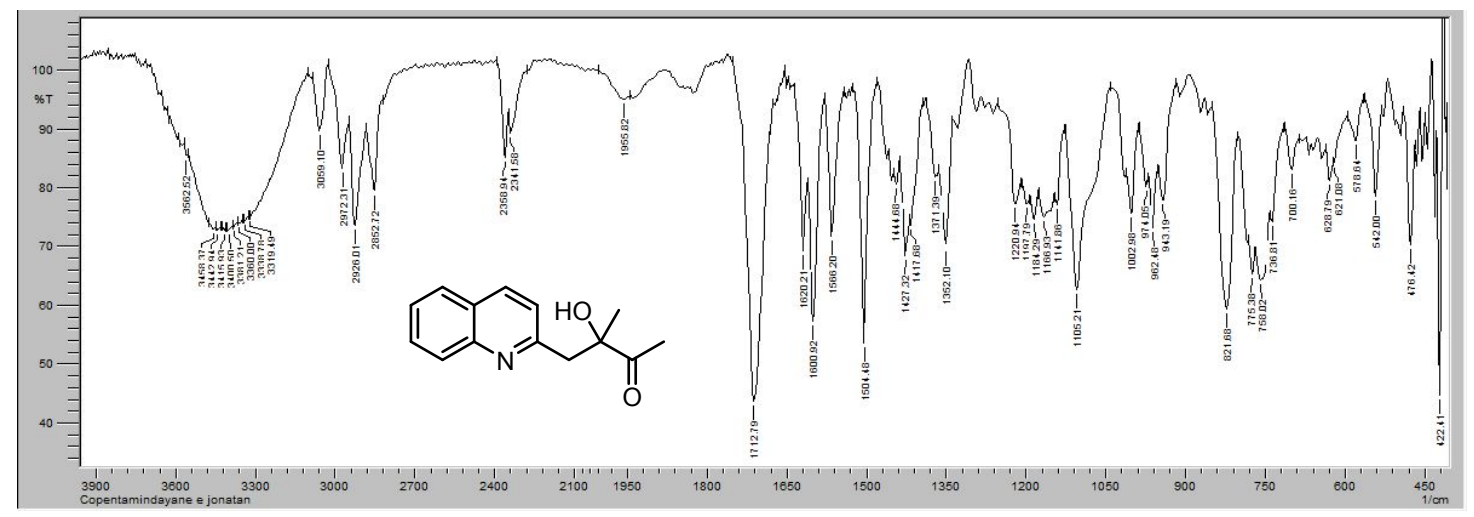

Figure S 61: IR - Prestigie-21 Fourier transform infrared spectrophotometer, SHIMADZU, with solids samples on $\mathrm{KBr}$ pellets of compound 3-hydroxy-3-methyl-4-(quinolin-2-yl)butan-2-one (3aa) 


\section{NOESY DATA}

\begin{tabular}{|c|c|}
\hline Acquisition Time (sec) $(0.2568,0.2568)$ & (mixing time $=550 \mathrm{~ms}$ ) \\
\hline 19 Feb $201907: 34: 18$ & \\
\hline UlvboxsrviXP-folderlc $p$ & IvboxsrviXP-folderlc pia de Epoxidos\Arlene Juliana $179 \backslash 400 \backslash$ pdatal $12 \pi$ \\
\hline Frequency $(\mathrm{MHz})$ & Nucleus \\
\hline Original Points Count $(1024,1024)$ & Points Count \\
\hline Solvent $\quad . \mathrm{CDCl} 3$ & Sweep Width $(\mathrm{Hz})$ \\
\hline Temperature (degree $C$ ) 0.000 & \\
\hline
\end{tabular}

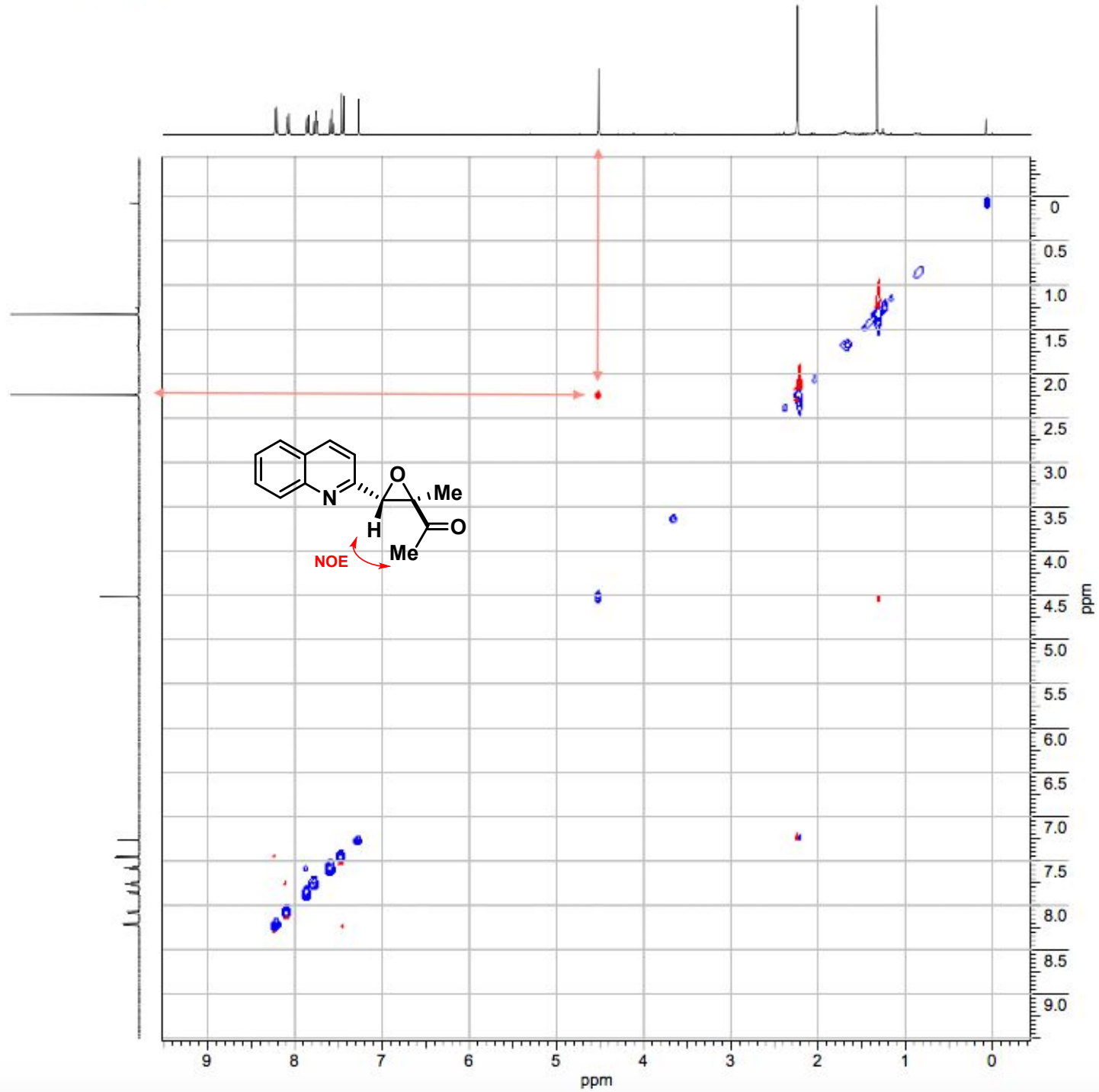

Figure S 62: NOESY of compound 1-(2-methyl-3-(quinolin-2-yl)oxiran-2-yl)ethan-1-one (5) 


\begin{tabular}{|c|c|c|c|c|}
\hline Acquisition Time (sec & $(0.2568,0.2568)$ & Comment & (mixing time $=550 \mathrm{~m}$ & \\
\hline Date & \multicolumn{3}{|c|}{18 Feb $201909: 57: 26$} & \\
\hline File Name & \multicolumn{4}{|c|}{ 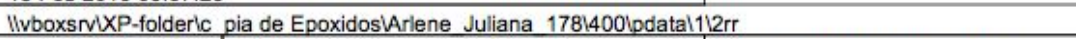 } \\
\hline Frequency $(\mathrm{MHz})$ & $(400.13,400.13)$ & Nucleus & $(1 \mathrm{H}, 1 \mathrm{H})$ & Number of Transients 16 \\
\hline Original Points Count & $(1024,1024)$ & Points Count & $(1024,1024)$ & Pulse Sequence \\
\hline Solvent & $\mathrm{CDCl} 3$ & Sweep Width $(\mathrm{Hz})$ & $(3987.24,3987.24)$ & Temperature (degree C) 25.001 \\
\hline
\end{tabular}

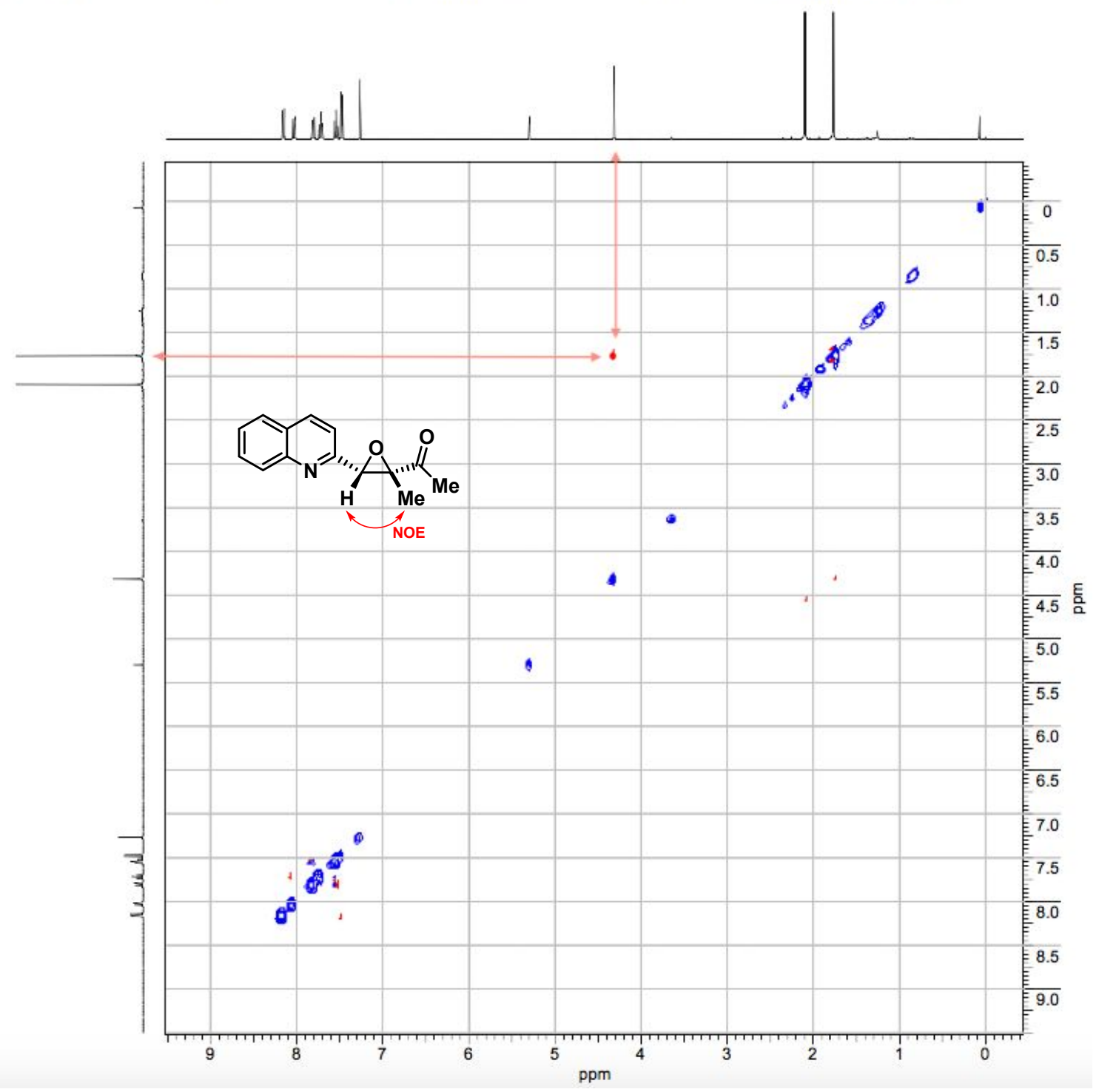

FigureS63: NOESY of compound 1-(2-methyl-3-(quinolin-2-yl)oxiran-2-yl)ethan-1-one (5') 


\section{DEUTERIUM-LABELING EXPERIMENTS}
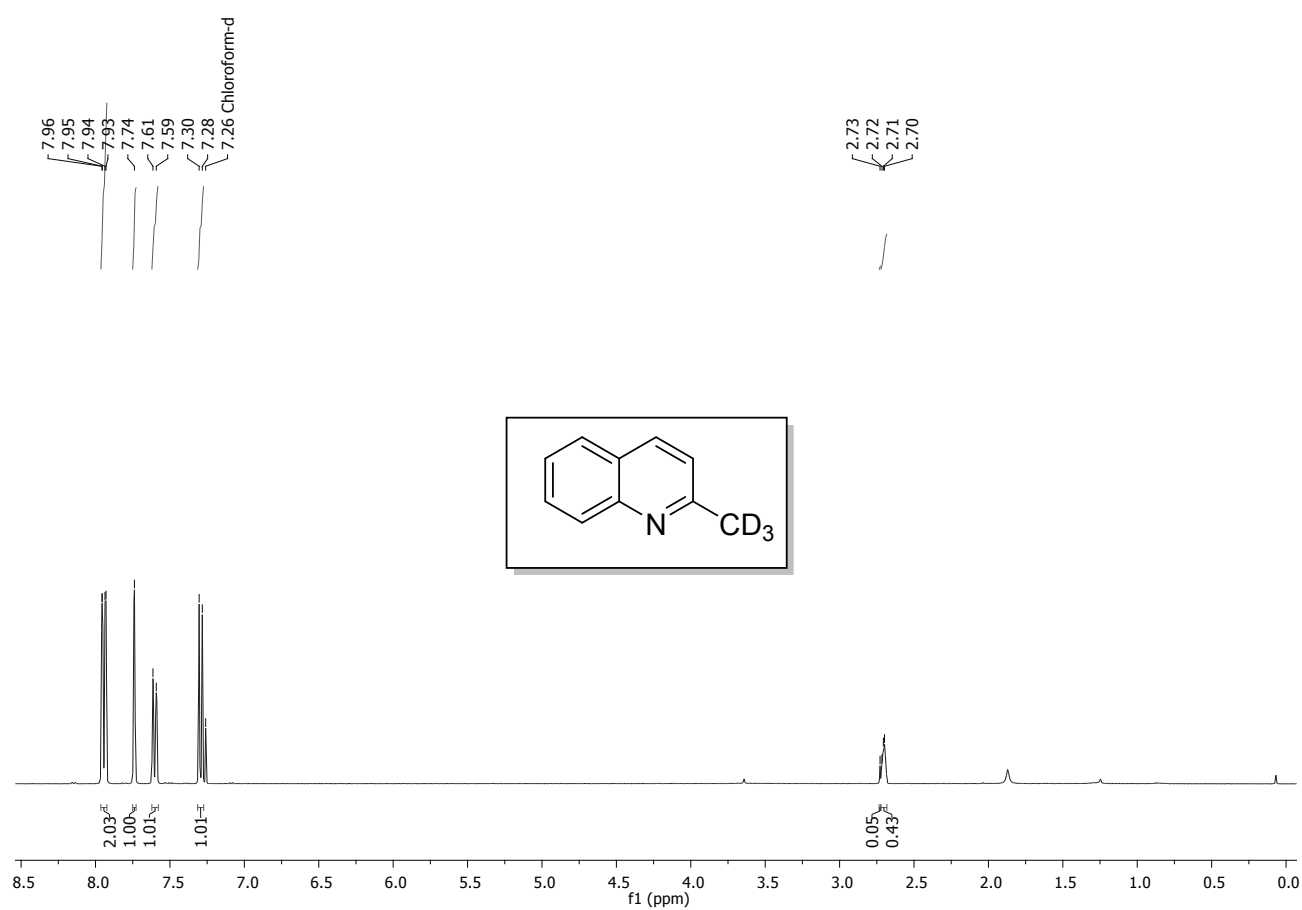

Figure S 64: ${ }^{1} \mathrm{H}$ NMR (400 MHz, $\mathrm{CDCl}_{3}$ ) of compound 1c-d $\mathbf{3}(85 \%-\mathrm{D})$.
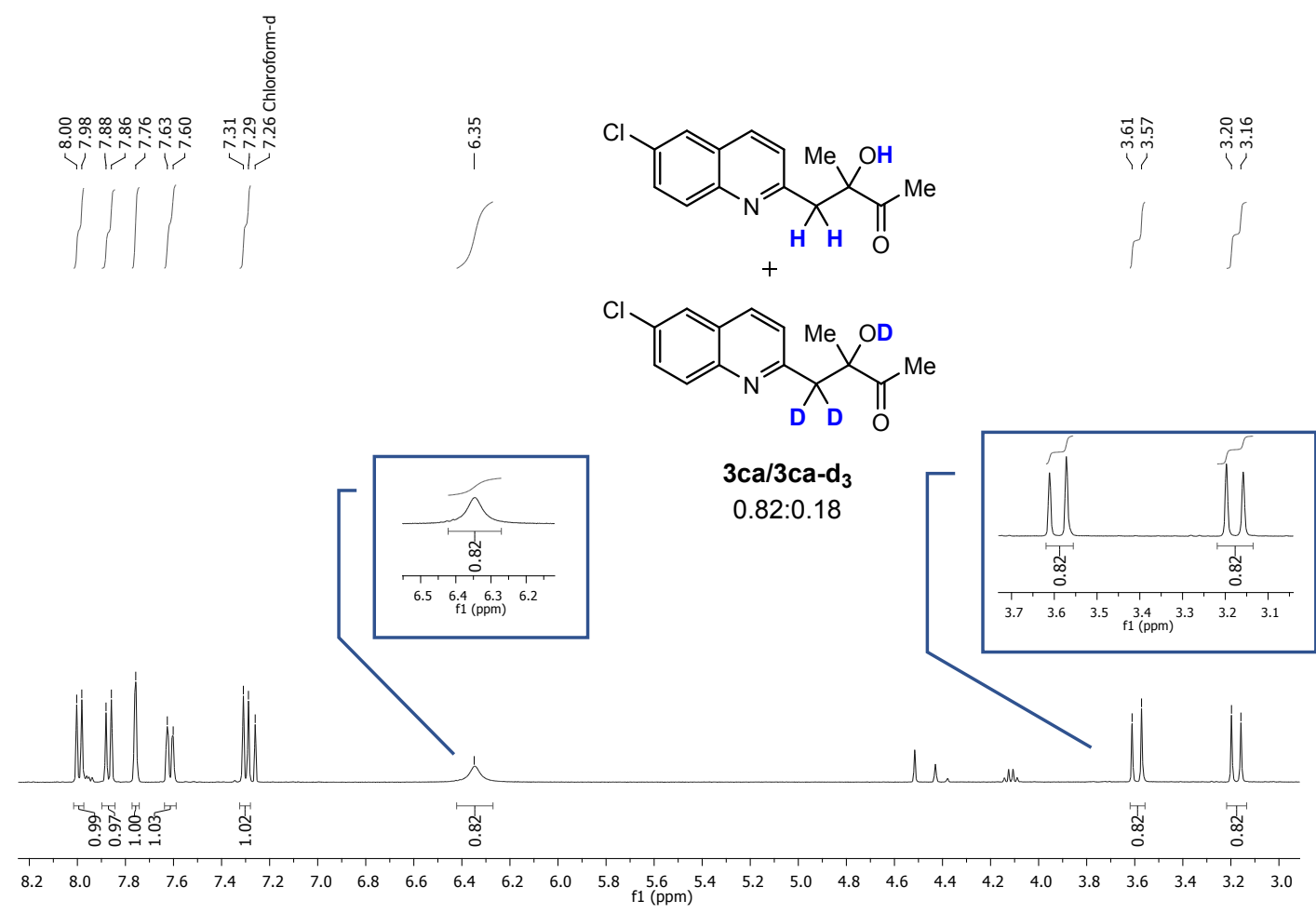

Figure $\mathrm{S} 65:{ }^{1} \mathrm{H}$ NMR $\left(400 \mathrm{MHz}, \mathrm{CDCl}_{3}\right)$ of isolated product mixture $\mathbf{3} \mathbf{c a}$ and $\mathbf{3} \mathbf{c a}-\mathbf{d}_{\mathbf{3}}$. 

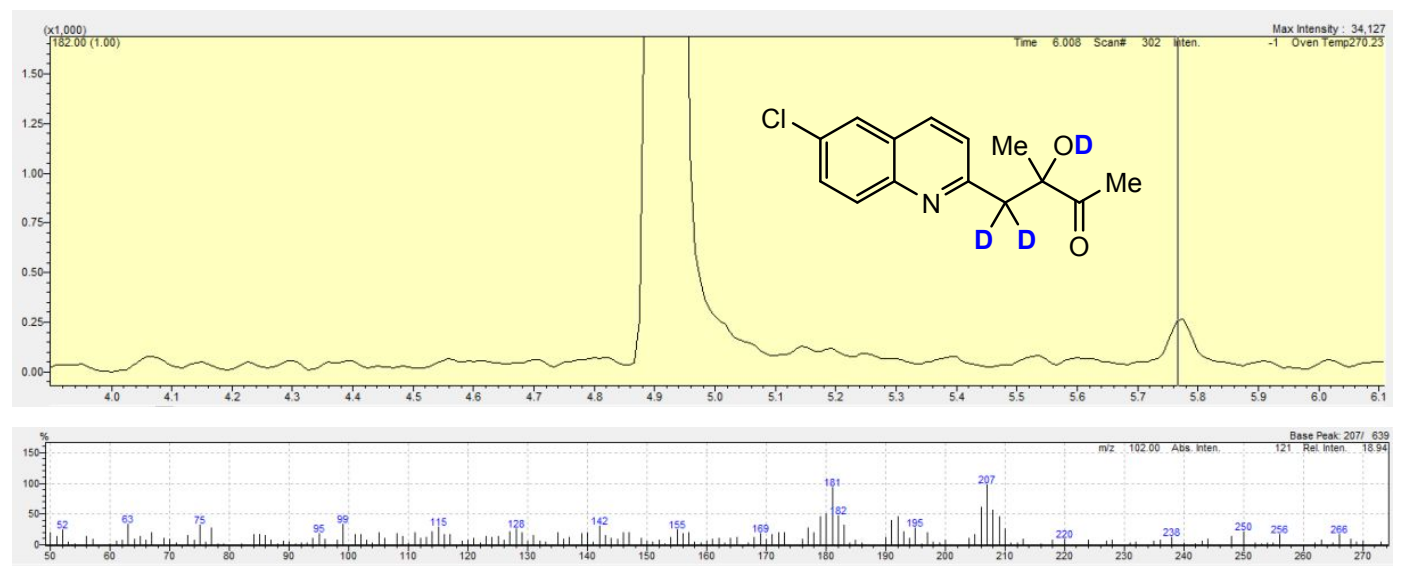

Figure S 66: GC-MS of the deuterated product $3 \mathrm{ca}^{\mathrm{d}} \mathrm{d}_{3}$ with $\mathrm{m} / \mathrm{z}=266$.

8. TRAPPING EXPERIMENT: MS ANALYSIS (Direct infusion-mass spectrometry (DI-MS) method in a mass spectrometer (Xevo TQD from Waters ${ }^{\mathrm{TM}}$ )

Initially, a full scan spectrum of the trapping experiment reaction after few hours of the beginning in the chosen mass range revealed all relevant peaks to probe the adduct formation between triplet diacetyl and TEMPO $(\mathrm{m} / \mathrm{z}=244)$ (Figure S67). Next, a detailed analysis using daughter ion scan of a parent or precursor $(\mathrm{m} / \mathrm{z}=243)$ furnished all the daughter ions resulting from fragmentation of this ion (Figure S68). Fragmentations related with McLafferty rearrangement ${ }^{6}$ type furnished the ion $\mathrm{m} / \mathrm{z}=20,1$ providing further insights of the radical intermediates.

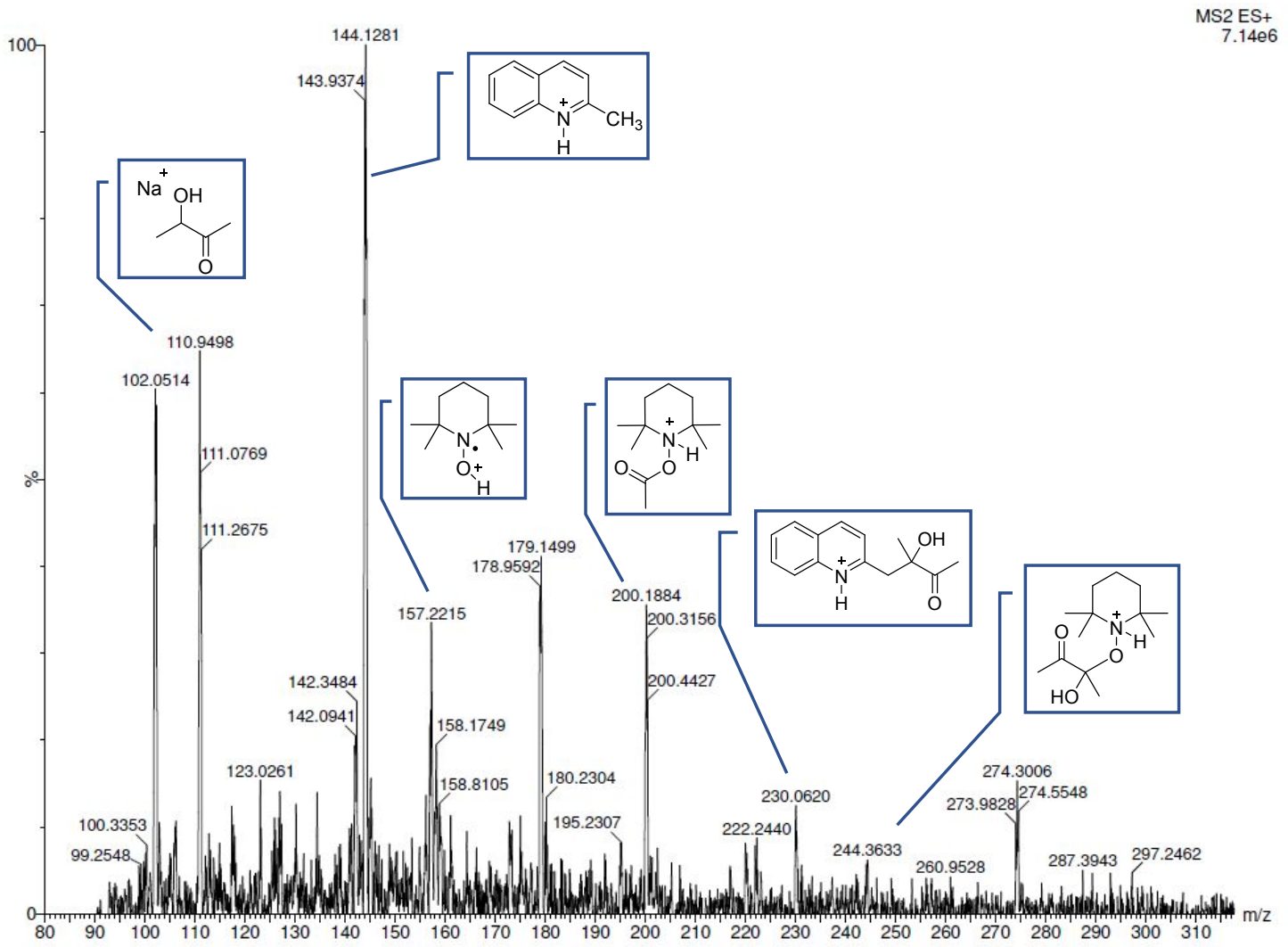

Figure S 67: Full scan chromatogram of the trapping experiment reaction after few hours of the beginning in the chosen mass range which all peaks that appear swept past the detector slit. 


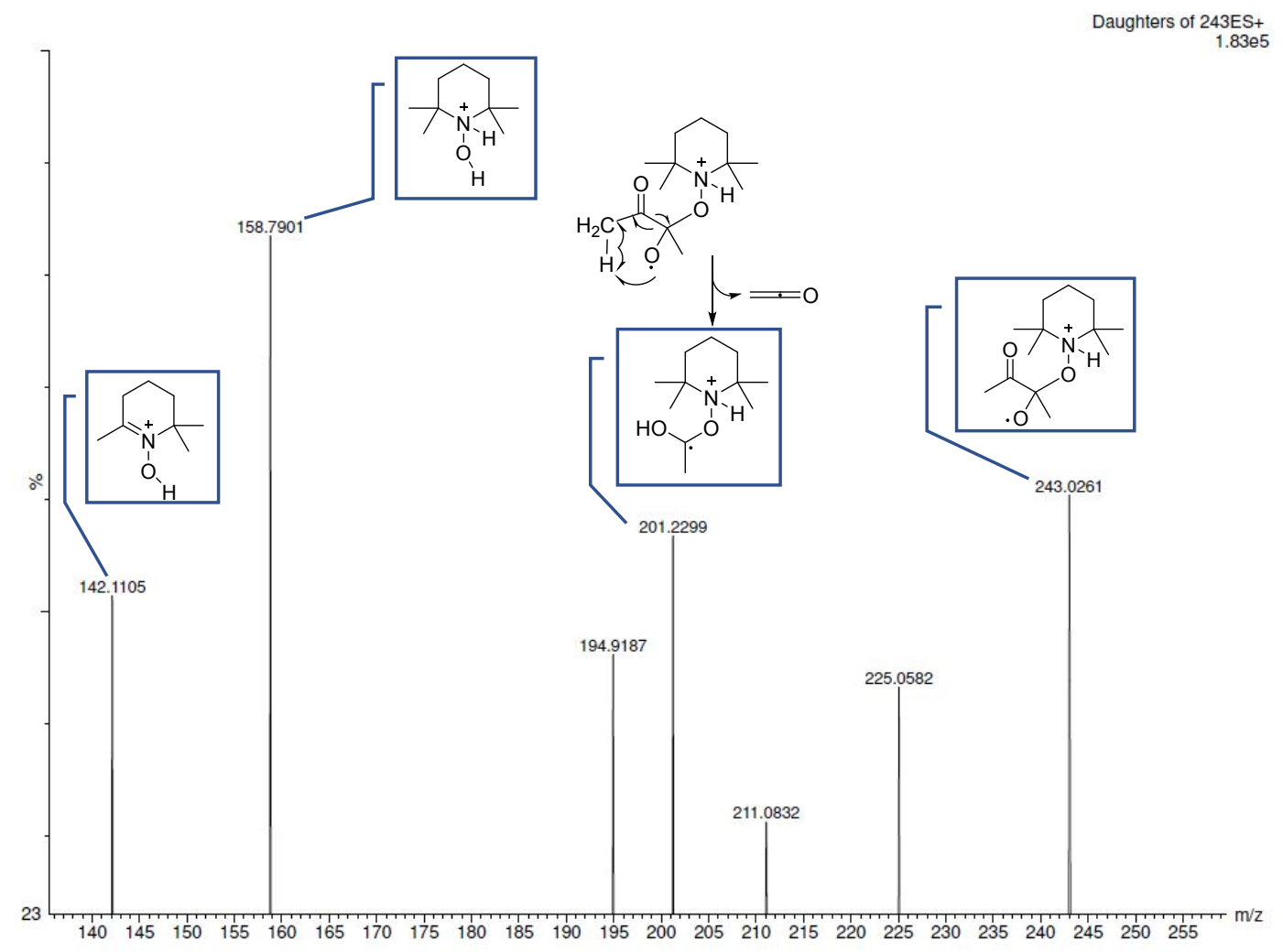

Figure S 68: Daughter ion scan chromatogram of a parent or precursor $(\mathrm{m} / \mathrm{z}=243)$ selected, obtaining all the daughter ions resulting from fragmentation of this ion. Observed fragmentations of TEMPO were previous described by Jahangiri and co-workers ${ }^{7}$. 


\section{THEORICAL MECHANISTIC STUDIES}

Table S2: Energies (E), ZPE and Gibbs (G) correction of starting materials, transition states, intermediates and products.

\begin{tabular}{|c|c|c|c|c|c|c|c|}
\hline \multicolumn{8}{|c|}{ (U)B3LYP/ 6-31+G(d,p) } \\
\hline Names & $E(a u)$ & $\mathrm{ZPC}_{\text {corr }}(\mathrm{au})$ & $\mathbf{G}_{\text {corr }}(\mathrm{au})$ & $\begin{array}{l}\left.<S^{2}\right\rangle \text { before } \\
\text { annihilation }\end{array}$ & $\begin{array}{c}<S^{2}>\text { after } \\
\text { annihilation }\end{array}$ & $\begin{array}{l}\text { Frequency } \\
\qquad\left(\mathrm{cm}^{-1}\right)\end{array}$ & G (au) \\
\hline diacetyl & -306.512516 & 0.092958 & 0.061277 & - & - & - & -306.451239 \\
\hline diacetyl (triplet) & -306.435333 & 0.091020 & 0.058444 & 2.01 & 2.00 & - & -306.376889 \\
\hline МeCHO & -153.852378 & 0.055406 & 0.030444 & - & - & - & -153.821934 \\
\hline $1 a$ & -441.298906 & 0.162683 & 0.128988 & - & - & - & -441.169918 \\
\hline $3 a$ & -747.825551 & 0.261170 & 0.217463 & & & - & -747.608088 \\
\hline $4 a$ & -593.954626 & 0.199667 & 0.160672 & - & - & - & -593.793954 \\
\hline 8 (doublet) & -440.645527 & 0.149709 & 0.116431 & 0.78 & 0.75 & - & -440.529096 \\
\hline 9 (doublet) & -307.093739 & 0.104372 & 0.07242 & 0.76 & 0.75 & - & -307.021319 \\
\hline 10 (doublet) & -747.156261 & 0.247353 & 0.202264 & 0.76 & 0.75 & - & -746.953997 \\
\hline 11 (doublet) & -153.199443 & 0.043022 & 0.017335 & 0.75 & 0.75 & - & -153.182108 \\
\hline 12 (doublet) & -594.516306 & 0.208874 & 0.168010 & 0.78 & 0.75 & - & -594.348296 \\
\hline 13 & -307.730251 & 0.117186 & 0.086305 & - & - & - & -307.643946 \\
\hline $\mathrm{TS}_{\mathrm{T}}$ (triplet) & -747.722209 & 0.250557 & 0.202954 & 2.02 & 2.00 & -1383.2618 & -747.519255 \\
\hline $\mathrm{TS2}_{\mathrm{T}}$ (triplet) & -747.700165 & 0.254514 & 0.209497 & 2.03 & 2.00 & -989.9151 & -747.490668 \\
\hline TS3 (doublet) & -748.354006 & 0.263099 & 0.218099 & 0.77 & 0.75 & -1407.5876 & -748.135907 \\
\hline TS4 $_{D}$ (doublet) & -747.147282 & 0.245754 & 0.201633 & 0.77 & 0.75 & -465.5396 & -746.945649 \\
\hline TS5 $_{D}$ (doublet) & -1188.448429 & 0.407270 & 0.348126 & 0.76 & 0.75 & -1407.5104 & -1188.100303 \\
\hline TS7 $_{\mathrm{T}}$ (triplet) & -306.398498 & 0.088212 & 0.054246 & 2.01 & 2.00 & -195.7281 & -306.344252 \\
\hline TS8 D $_{\text {(doublet) }}$ & -594.492889 & 0.206850 & 0.165527 & 0.77 & 0.75 & -328.0893 & -594.327362 \\
\hline TS9 ${ }_{D}$ (doublet) & -901.014282 & 0.299055 & 0.248478 & 0.79 & 0.75 & -1827.4552 & -900.765804 \\
\hline
\end{tabular}


Table S3: Calculated reaction pathways (Paths A-C) disclosed in the main text.

\begin{tabular}{|c|c|c|c|c|}
\hline initiation & $E(a u)$ & $\begin{array}{c}\Delta \mathrm{E}_{\mathrm{rel}} \\
\left(\mathrm{kcal}^{\left.-\mathrm{mol}^{-1}\right)}\right.\end{array}$ & G (au) & $\begin{array}{c}\Delta \mathrm{G}_{\text {rel }} \\
\left(\mathrm{kcal}^{\left.-\mathrm{mol}^{-1}\right)}\right.\end{array}$ \\
\hline diacetyl + 1 & -747.811423 & 0.0 & -747.621158 & 0.0 \\
\hline diacetyl-T + 1 & -747.734240 & 48.4 & -747.546808 & 46.7 \\
\hline \multicolumn{5}{|l|}{ Path-A } \\
\hline diacetyl-T + 1 & -747.734240 & 0.0 & -747.546808 & 0.0 \\
\hline $\mathrm{TS}_{\mathrm{T}}$ & -747.722209 & 7.5 & -747.519255 & 17.3 \\
\hline $8+9$ & -747.739266 & -3.2 & -747.550415 & -2.3 \\
\hline $3 a$ & -747.825551 & -57.3 & -747.608088 & -38.5 \\
\hline \multicolumn{5}{|l|}{ Path-B-1 } \\
\hline diacetyl-T + 1 + 1 & -1189.033146 & 0.0 & -1188.716726 & 0.0 \\
\hline $\mathrm{TS} 1_{\mathrm{T}}+1$ & -1189.021115 & 7.5 & -1188.689173 & 17.3 \\
\hline $8+9+1$ & -1189.038172 & -3.2 & -1188.720333 & -2.3 \\
\hline $8+\mathrm{TS}_{\mathrm{D}}$ & -1188.999533 & 21.1 & -1188.665003 & 32.5 \\
\hline $8+8+13$ & -1189.021305 & 7.4 & -1188.702138 & 9.2 \\
\hline \multicolumn{5}{|l|}{ Path-B-2 } \\
\hline $\begin{array}{c}\text { diacetyl-T + } 1+ \\
\text { diacetyl +1 }\end{array}$ & -1495.545662 & 0.0 & -1495.167965 & 0.0 \\
\hline $\mathrm{TS}_{\mathrm{T}}+$ diacetyl + 1 & -1495.533632 & 7.5 & -1495.140413 & 17.3 \\
\hline $8+9+$ diacetyl + 1 & -1495.550689 & -3.2 & -1495.171573 & -2.3 \\
\hline $9+\mathrm{TS}_{\mathrm{D}}+1$ & -1495.539928 & 3.6 & -1495.136887 & 19.5 \\
\hline $9+10+1$ & -1495.548907 & -2.0 & -1495.145235 & 14.3 \\
\hline 3 + diacetyl + 1 & -1495.636974 & -57.3 & -1495.229246 & -38.5 \\
\hline $9+$ TS5 $_{D}$ & -1495.542168 & 2.2 & -1495.121622 & 29.1 \\
\hline $3+8+9$ & -1495.564817 & -12.0 & -1495.158503 & 5.9 \\
\hline \multicolumn{5}{|l|}{ Path-C } \\
\hline $\begin{array}{c}\text { diacetyl-T + } 1+ \\
\text { diacetyl }\end{array}$ & -1054.246756 & 0.0 & -1053.998047 & 0.0 \\
\hline $\mathrm{TS7}_{\mathrm{T}}+1+$ diacetyl & -1054.209921 & 23.1 & -1053.965410 & 20.5 \\
\hline $11+11+1+$ diacetyl & -1054.210309 & 22.9 & -1053.985374 & 8.0 \\
\hline $\mathrm{TS}_{\mathrm{D}}+11+$ diacetyl & -1054.204848 & 26.3 & -1053.960709 & 23.4 \\
\hline $12+11+$ diacetyl & -1054.228265 & 11.6 & -1053.981643 & 10.3 \\
\hline $\mathrm{TS}_{\mathrm{D}}+11$ & -1054.213725 & 20.7 & -1053.947912 & 31.5 \\
\hline $4+11+9$ & -1054.247808 & -0.7 & -1053.997381 & 0.4 \\
\hline 4 + diacetyl + MeCHO & -1054.319520 & -45.7 & -1054.067127 & -43.3 \\
\hline
\end{tabular}


Table S4: Energies (E), ZPE and Gibbs (G) correction of starting materials, transition states, intermediates and products involving complexation of LiOCl 4 .

\begin{tabular}{|c|c|c|c|c|c|c|c|}
\hline \multicolumn{8}{|c|}{ (U)B3LYP/ 6-31+G(d,p) } \\
\hline Names & $E(a u)$ & $\mathrm{ZPC}_{\text {corr }}(\mathrm{au})$ & $\mathbf{G}_{\text {corr }}(\mathrm{au})$ & $\begin{array}{l}\left\langle\mathrm{S}^{2}\right\rangle \text { before } \\
\text { annihilation }\end{array}$ & $\begin{array}{c}\left\langle\mathrm{S}^{2}\right\rangle \text { after } \\
\text { annihilation }\end{array}$ & $\begin{array}{l}\text { Frequency } \\
\left(\mathrm{cm}^{-1}\right)\end{array}$ & G (au) \\
\hline diacetyl_ $\mathrm{LiClO}_{4}$ & -1074.916040 & 0.110912 & 0.065525 & - & - & - & -1074.850515 \\
\hline diacetyl_LiClO 4 (triplet) & -1074.838569 & 0.108948 & 0.063239 & 2.01 & 2.00 & - & -1074.775330 \\
\hline 1a_LiClO 4 & -1209.706057 & 0.182044 & 0.136918 & - & - & - & -1209.569139 \\
\hline 8_LiClO 4 (doublet) & -1209.054001 & 0.168667 & 0.124765 & 0.78 & 0.75 & - & -1208.929236 \\
\hline 10_LiClO 4 (doublet) & -1515.568088 & 0.265494 & 0.211376 & 0.76 & 0.75 & - & -1515.356712 \\
\hline $\mathrm{TS}_{\mathrm{T}} \mathrm{LiClO}_{4}$ (triplet) & -1516.129723 & 0.269545 & 0.213104 & 2.02 & 2.00 & -1716.68 & -1515.916619 \\
\hline $\mathrm{TS}_{\text {D_LiClO }}$ (doublet) & -1515.556457 & 0.263606 & 0.208129 & 0.77 & 0.75 & -414.78 & -1515.348328 \\
\hline $\mathrm{TS}_{D_{\mathrm{D}} \mathrm{LiClO}_{4}}$ (doublet) & -1956.871825 & 0.428372 & 0.36315 & 0.76 & 0.75 & -1529.87 & -1956.508675 \\
\hline
\end{tabular}


Table S5: Calculated reaction pathways (Paths A-B) involving complexation of $\mathrm{LiOCl}_{4}$.

\begin{tabular}{|c|c|c|c|c|}
\hline initiation & $E(a u)$ & $\begin{array}{c}\Delta \mathrm{E}_{\text {rel }} \\
\left(\mathrm{kcal}^{\left.-\mathrm{mol}^{-1}\right)}\right.\end{array}$ & G (au) & $\begin{array}{c}\Delta \mathbf{G}_{\mathrm{rel}} \\
\left(\mathrm{kcal}^{\mathrm{mol}} \mathrm{mol}^{-1}\right)\end{array}$ \\
\hline diacetyl_LiClO 4 + 1 & -1074.916040 & 0.0 & -1074.850515 & 0.0 \\
\hline diacetyl-T_LiClO ${ }_{4}+1$ & -1074.838569 & 48.6 & -1074.775330 & 47.2 \\
\hline \multicolumn{5}{|l|}{ Path-A } \\
\hline diacetyl-T + 1_LiClO & -1516.141391 & 0.0 & -1515.946029 & 0.0 \\
\hline $\mathrm{TS1}_{\mathrm{T}} \mathrm{LiClO}_{4}$ & -1516.129723 & 7.3 & -1515.916619 & 18.5 \\
\hline 8_ $\mathrm{LiClO}_{4}+9$ & -1516.147740 & -4.0 & -1515.950555 & -2.8 \\
\hline \multicolumn{5}{|l|}{ Path-B-2 } \\
\hline $\begin{array}{c}\text { diacetyl-T + 1 + } \\
\text { diacetyl_LiClO }{ }_{4}+1\end{array}$ & -2263.949186 & 0.0 & -2263.567241 & 0.0 \\
\hline $\begin{array}{c}8+9+ \\
\text { diacetyl_LiClO } \\
4+1\end{array}$ & -2263.954212 & -3.2 & -2263.570848 & -2.3 \\
\hline $9+\mathrm{TS}_{\mathrm{D}_{-} \mathrm{LiClO}_{4}}+1$ & -2263.949103 & 0.05 & -2263.539566 & 17.4 \\
\hline $9+1+10$ LiClO $_{4}$ & -2263.960734 & -7.2 & -2263.547950 & 12.1 \\
\hline $9+10+1 \_\mathrm{LiClO}_{4}$ & -2263.956058 & -4.3 & -2263.544456 & 14.3 \\
\hline $9+\mathrm{TS}_{\mathrm{D}_{-} \mathrm{LiClO}_{4}}$ & -2263.965565 & -10.3 & -2263.529995 & 23.4 \\
\hline $9+3+8 \mathrm{LiClO}_{4}$ & -2263.973291 & -15.1 & -2263.558643 & 5.4 \\
\hline
\end{tabular}




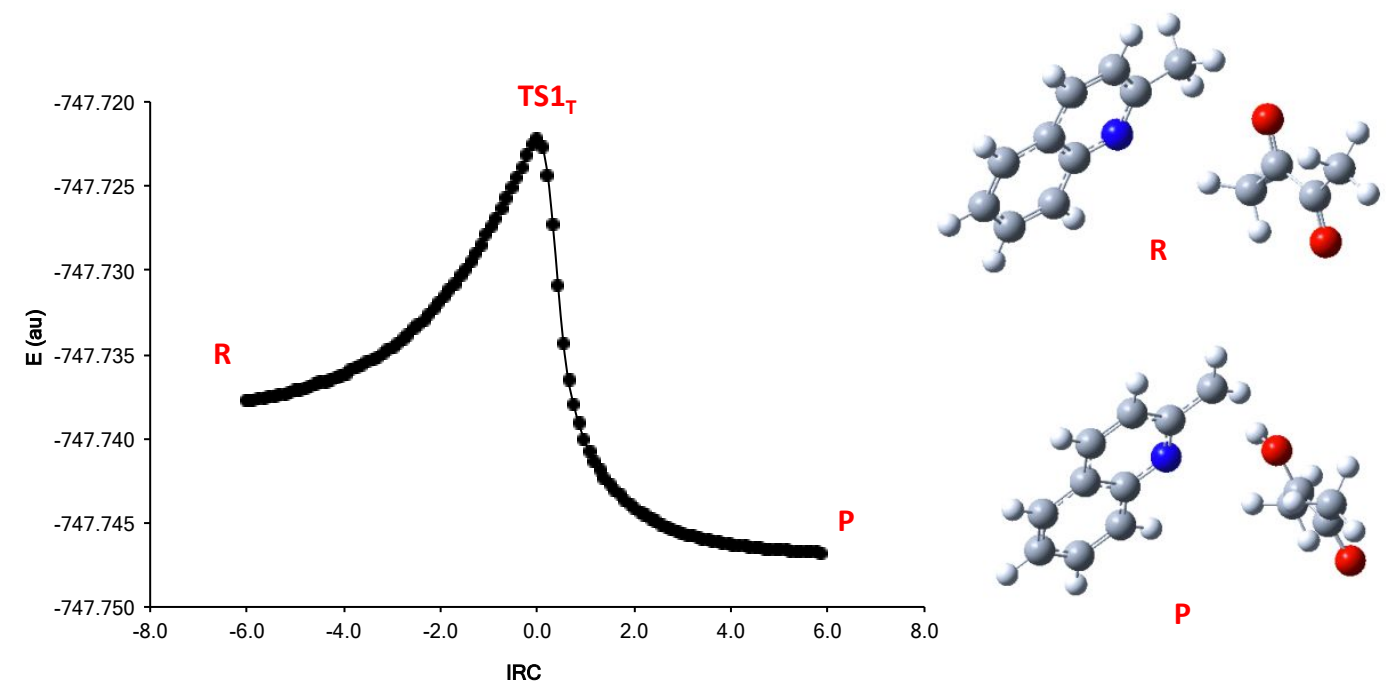

Figure S 69: Intrinsic Reactional Coordinates (IRC) for TS1 $1_{\mathrm{T}}$.
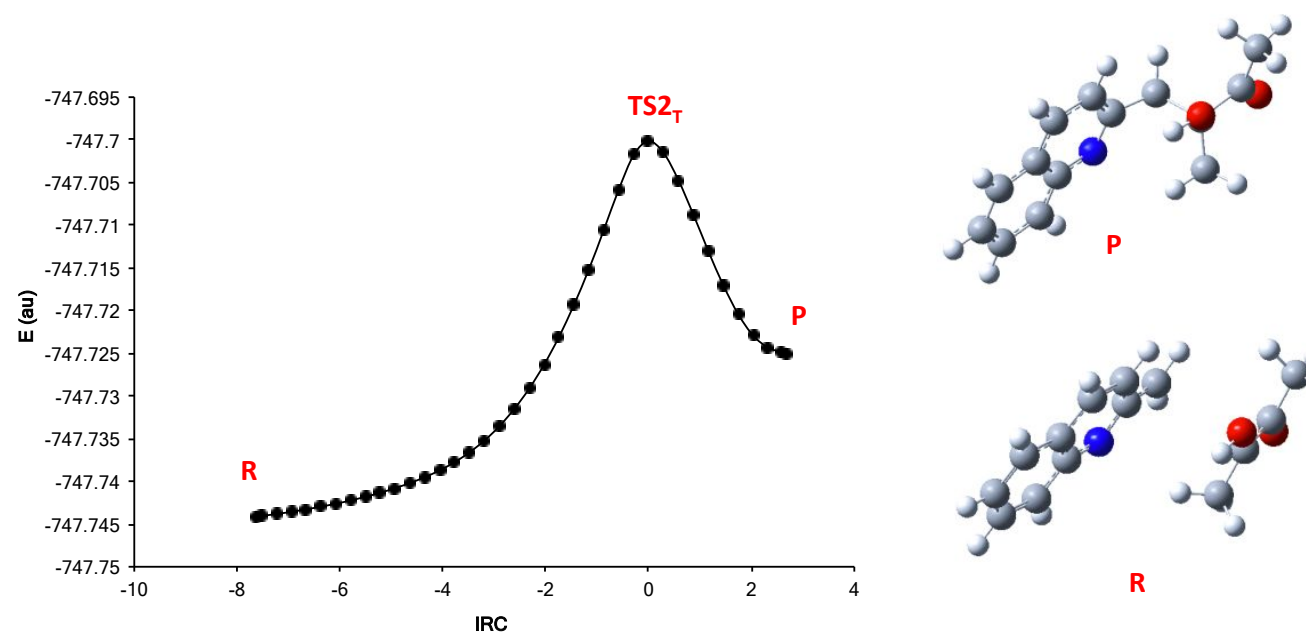

Figure S 70: Intrinsic Reactional Coordinates (IRC) for TS2 ${ }_{\mathrm{T}}$.
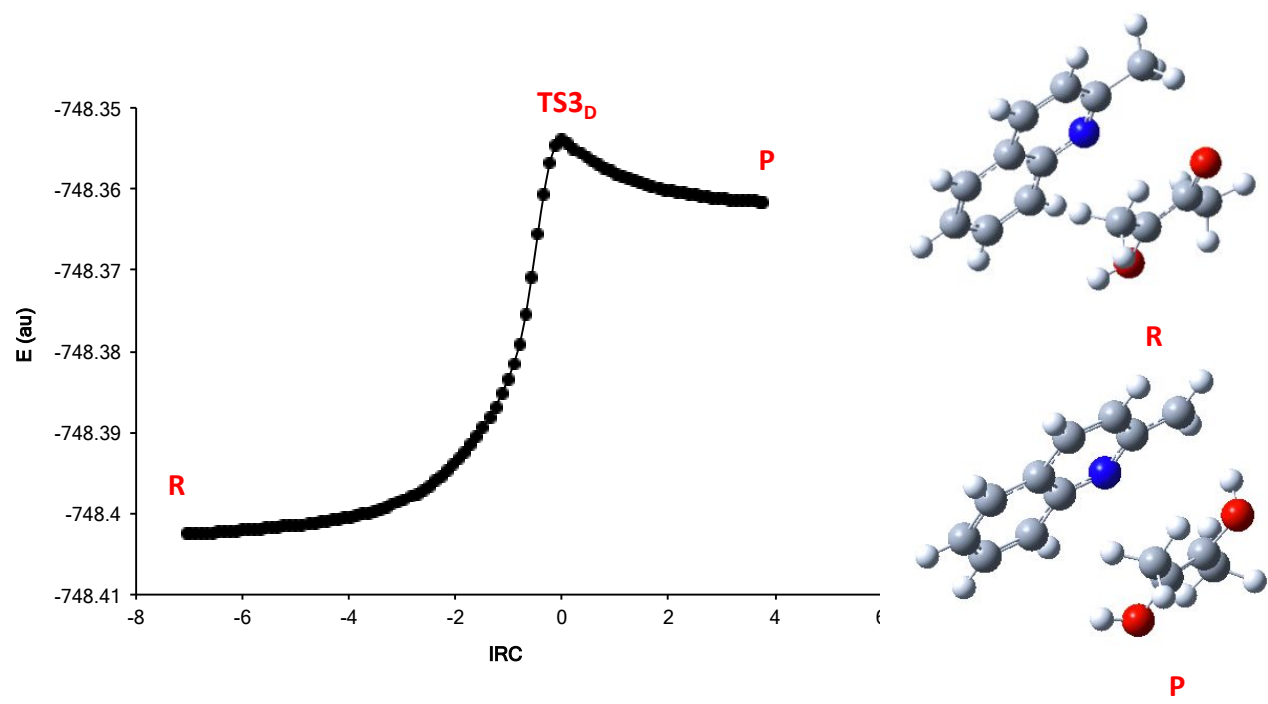

Figure S 71: Intrinsic Reactional Coordinates (IRC) for TS3 ${ }_{\mathrm{D}}$. 

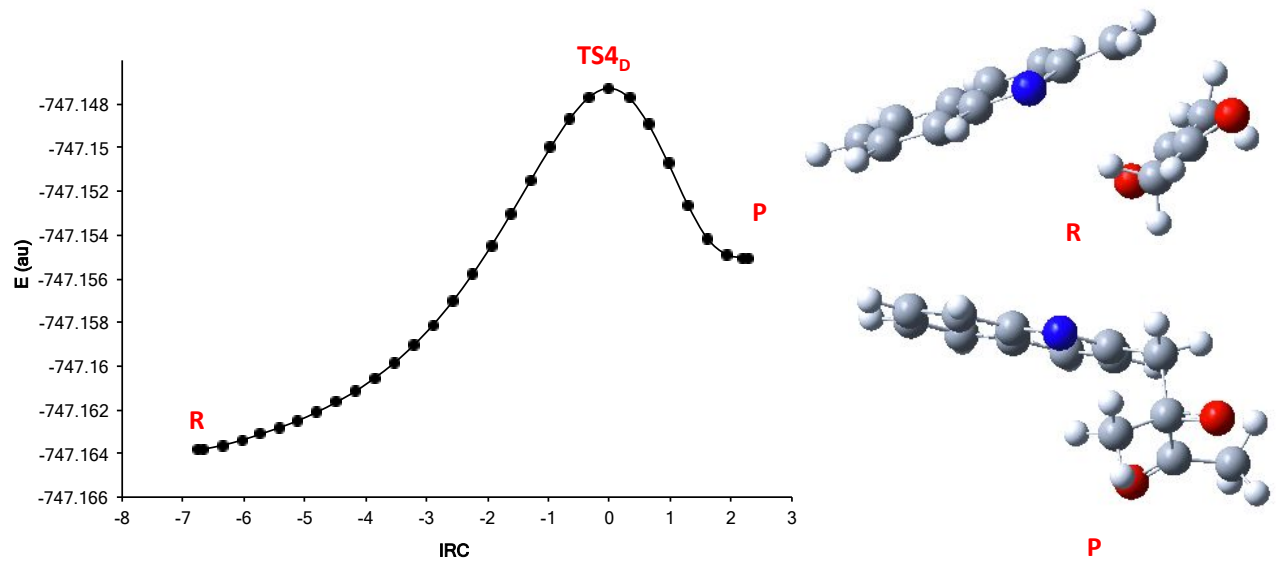

Figure S 72: Intrinsic Reactional Coordinates (IRC) for TS4 ${ }_{D}$.

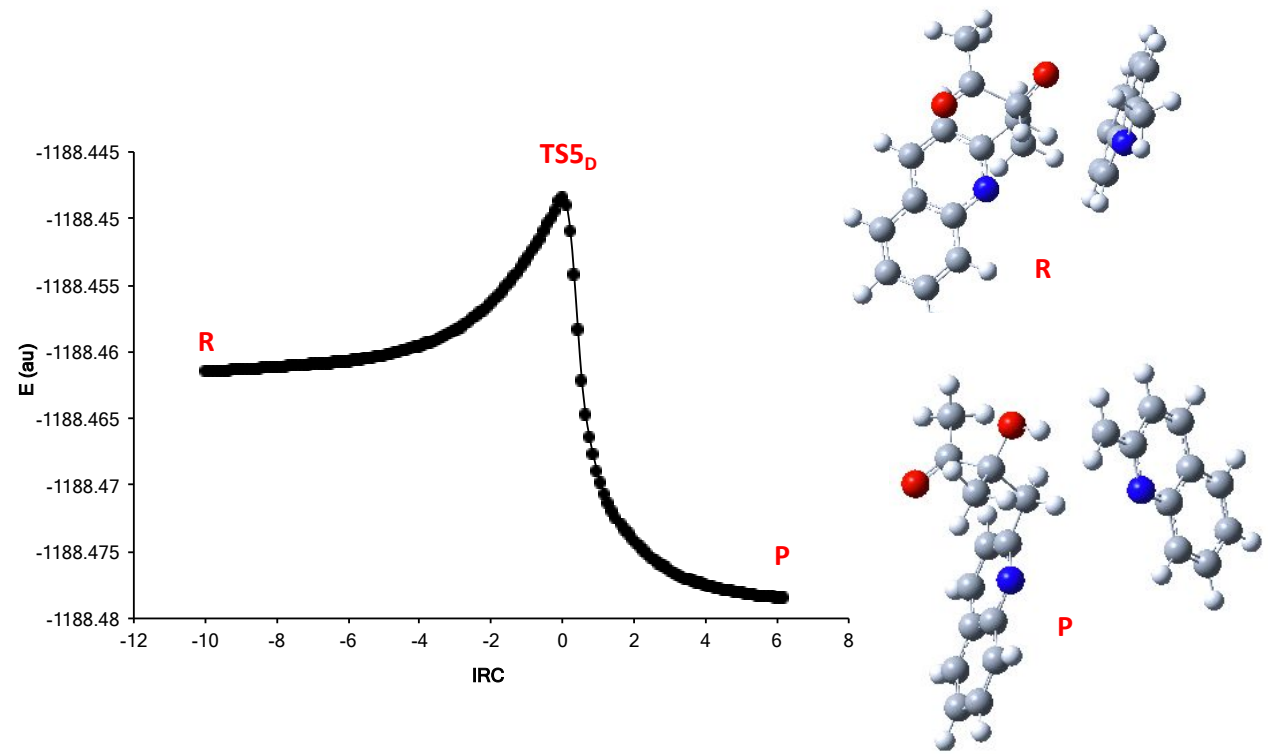

Figure S 73: Intrinsic Reactional Coordinates (IRC) for TS5 $5_{D}$.
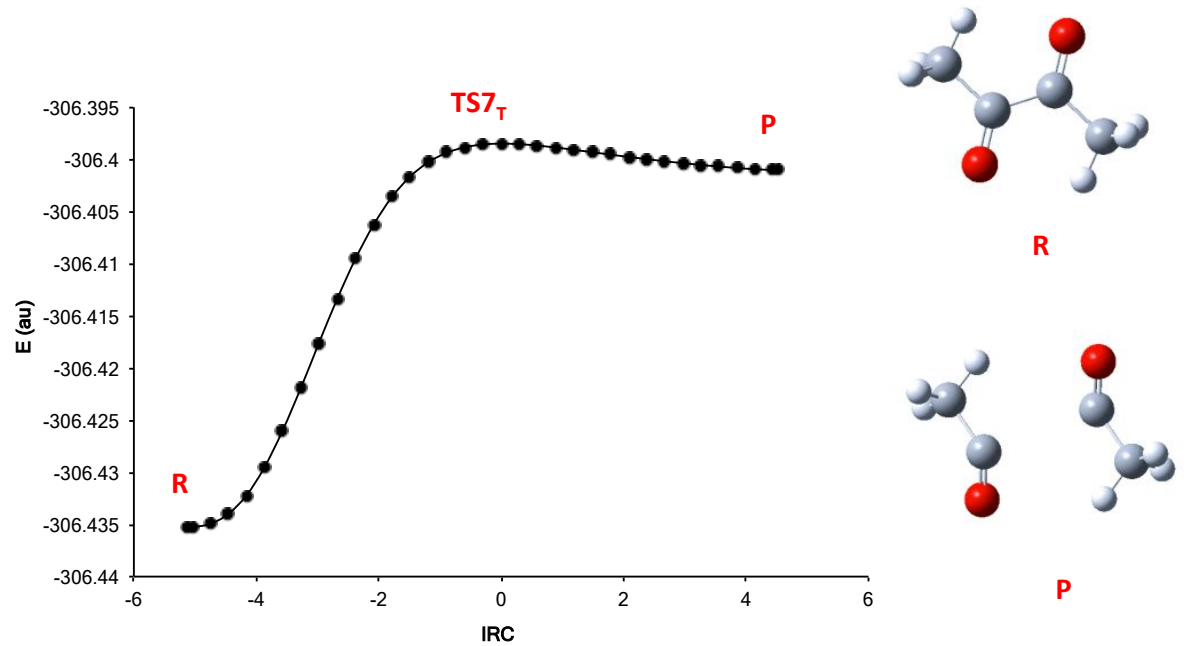

Figure S 74: Intrinsic Reactional Coordinates (IRC) for TS7 ${ }_{T}$. 

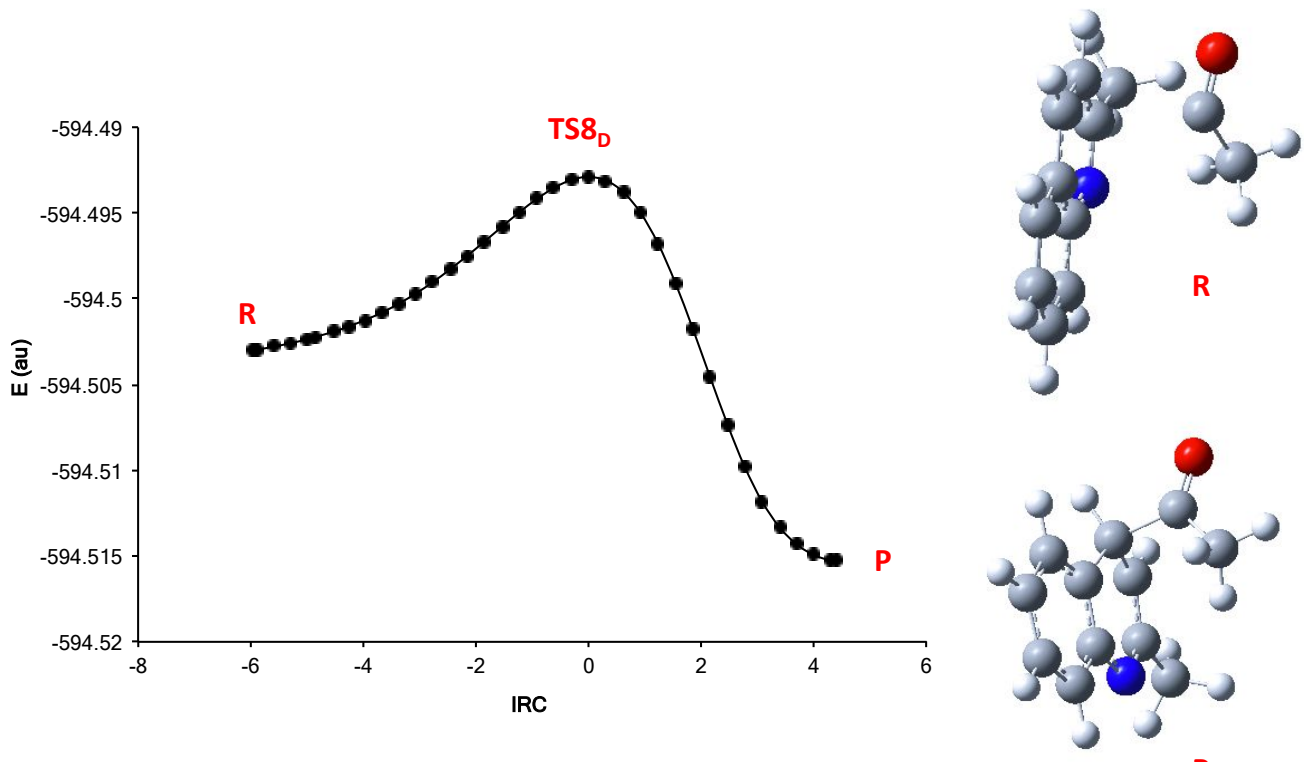

Figure S 75: Intrinsic Reactional Coordinates (IRC) for TS8 $8_{D}$.

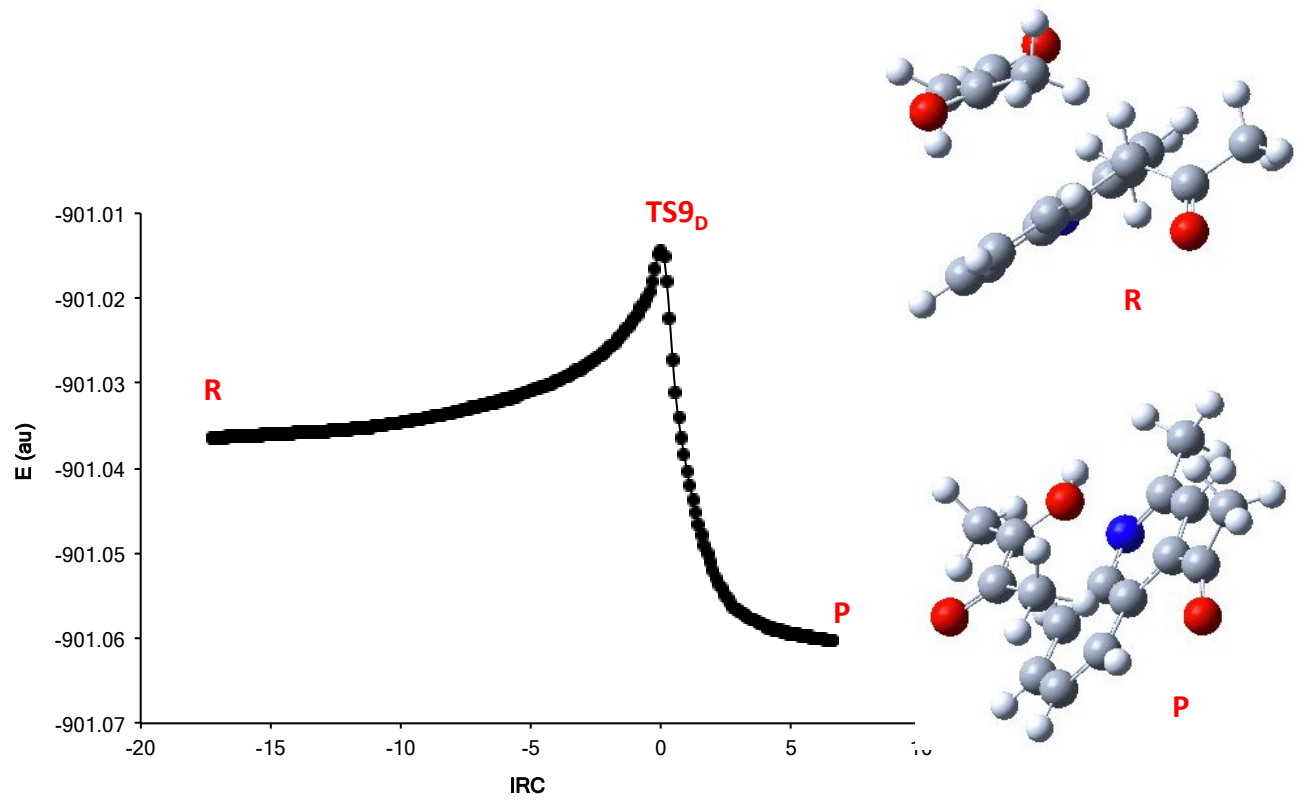

Figure S 76: Intrinsic Reactional Coordinates (IRC) for TS9 D. $^{\text {. }}$ 


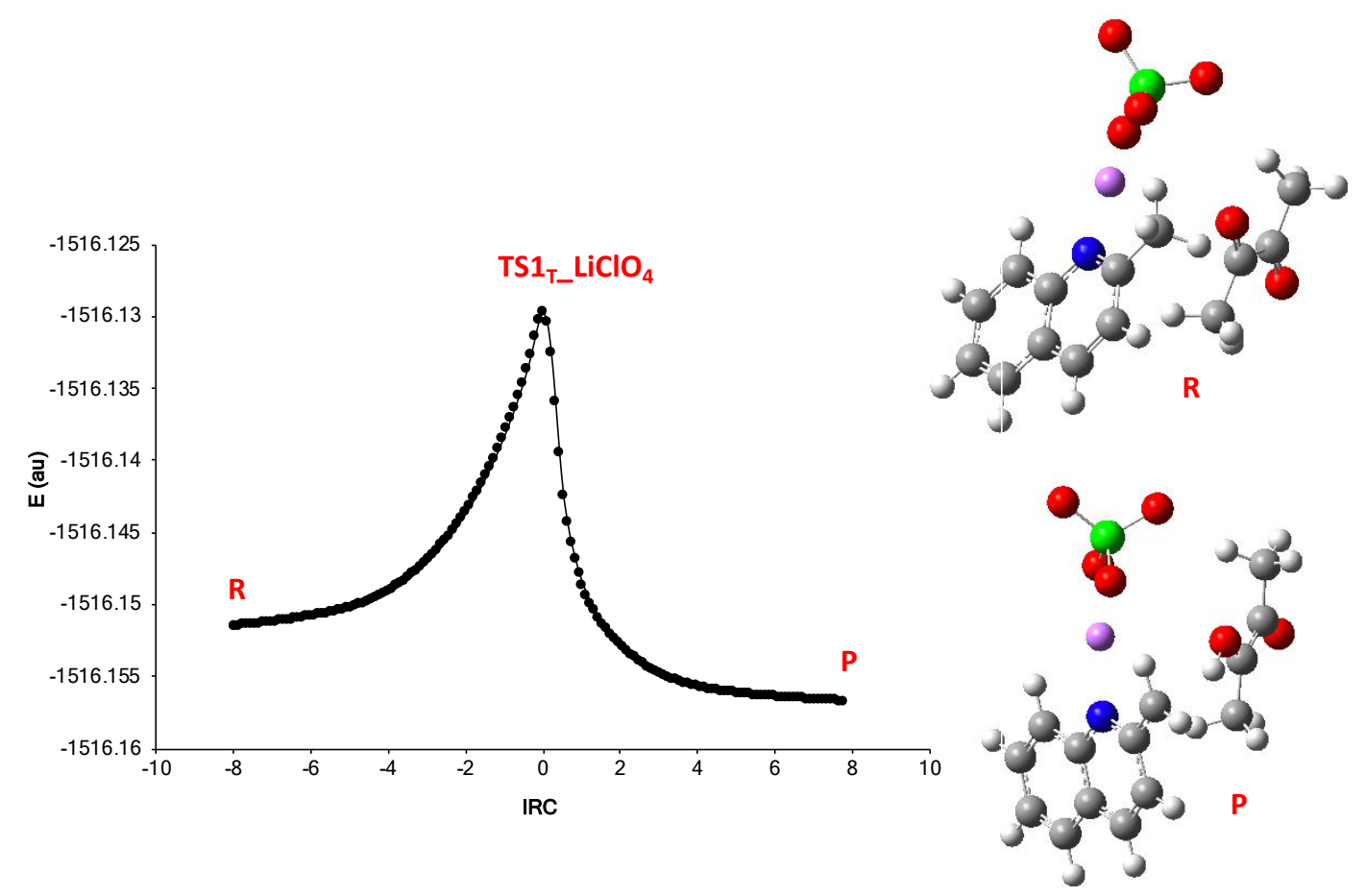

Figure S 77: Intrinsic Reactional Coordinates (IRC) for TS1 $\mathbf{T}_{\mathbf{T}} \mathbf{L i C l O}_{\mathbf{4}}$.

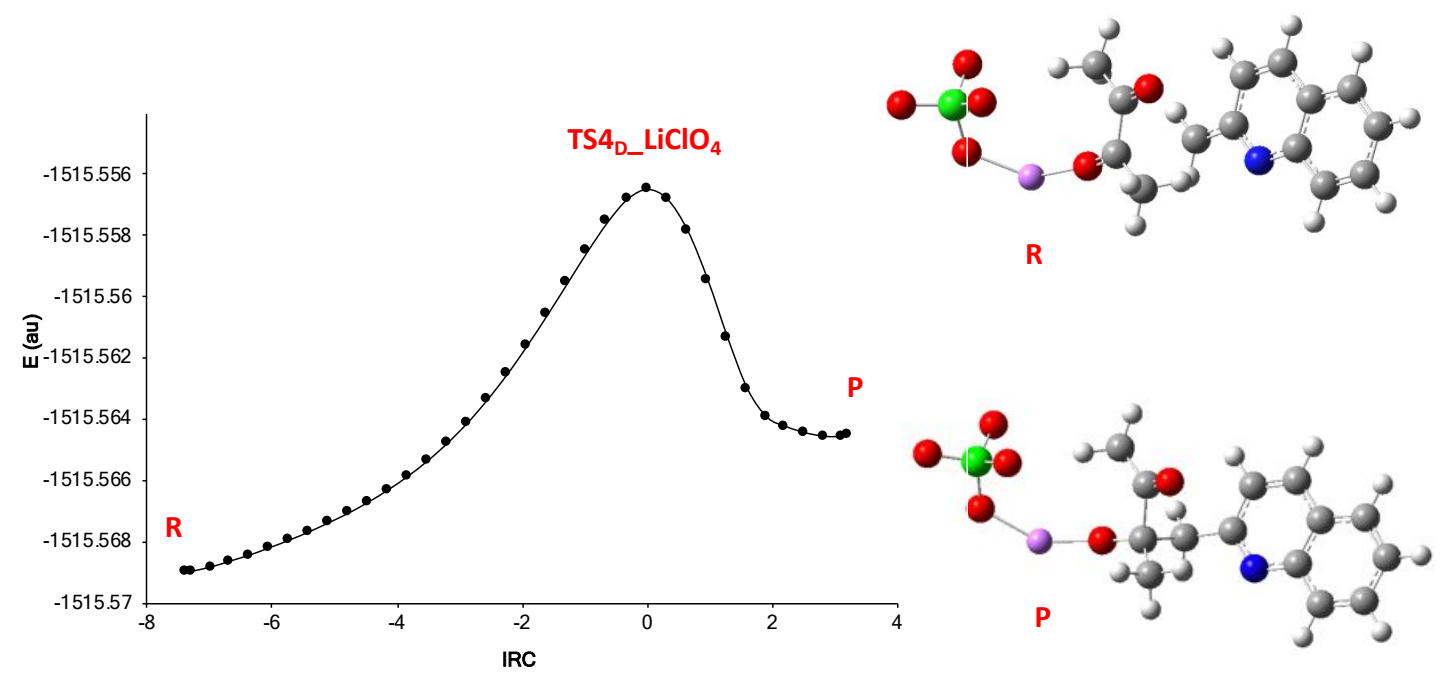

Figure S 78: Intrinsic Reactional Coordinates (IRC) for TS4 D_LiClO $_{4}$. 


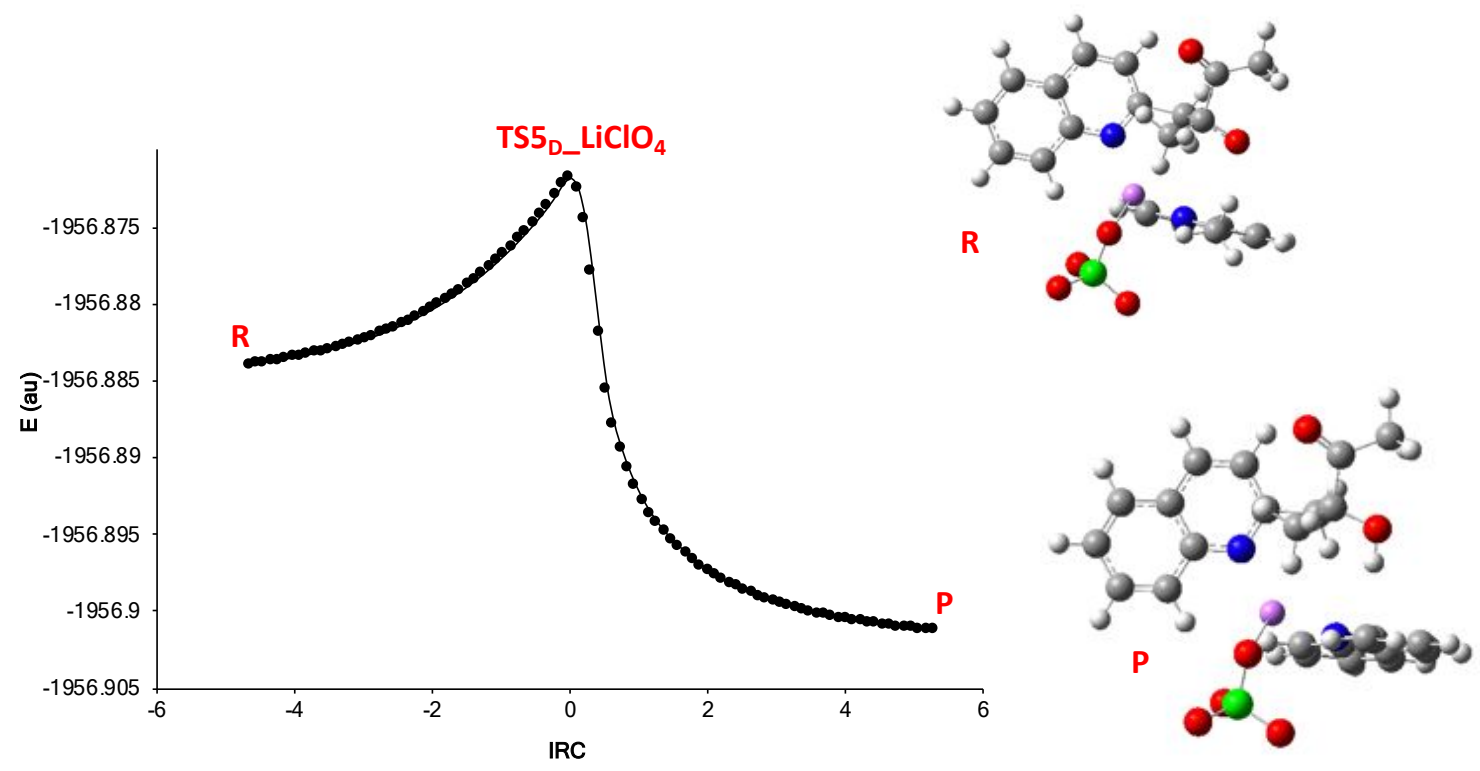

Figure S 79: Intrinsic Reactional Coordinates (IRC) for TS5 D_LiClO $_{4}$. 


\section{CARTESIAN COORDINATES}

\section{Diacetyl}

C

$\mathrm{H} \quad-0.64734-0.11928-0.7996$

$\begin{array}{lllll}\mathrm{H} & -2.3137 & 0.18740 & -0.22527\end{array}$

$\begin{array}{llll}\mathrm{H} & -1.47044 & -1.27507 & 0.28841\end{array}$

$\begin{array}{lllll}\mathrm{C} & -0.83427 & 0.50017 & 1.26885\end{array}$

$\begin{array}{llll}\mathrm{C} & -1.70776 & 0.45487 & 2.55746\end{array}$

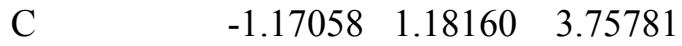

$\begin{array}{llll}\mathrm{H} & -0.18628 & 0.78287 & 4.02534\end{array}$

$\begin{array}{llll}\mathrm{H} & -1.01999 & 2.23907 & 3.51599\end{array}$

$\begin{array}{llll}\mathrm{H} & -1.86369 & 1.07718 & 4.59292\end{array}$

O $\quad-2.76645 \quad-0.15076 \quad 2.54353$

$\begin{array}{llll}\mathrm{O} & 0.21807 & 1.11696 & 1.30255\end{array}$

\section{MeCHO}

$\mathrm{C}$

$\mathrm{H}$

$\mathrm{H}$

$\mathrm{H}$

C

$\mathrm{H}$

$\mathrm{O}$

3a

$\mathrm{C}$

C

C

C

C

C

$\mathrm{H}$

$\mathrm{H}$

$\mathrm{C}$

$\mathrm{H}$

$\mathrm{H}$

C

C

$\mathrm{H}$

$\mathrm{H}$

C

$\mathrm{H}$

$\mathrm{H}$

C

C

$\mathrm{H}$

$\mathrm{H}$

$\mathrm{H}$

$\mathrm{O}$

$\mathrm{H}$

C

$\mathrm{O}$

C

$\mathrm{H}$ $\begin{array}{lll}-0.57018 & 0.61404 & 0.00000\end{array}$ $\begin{array}{lll}-0.21352 & -0.39477 & 0.00000\end{array}$ $\begin{array}{llll}-0.2135 & 1.11843 & -0.87365\end{array}$

$\begin{array}{lll}-1.64018 & 0.61405 & 0.00000\end{array}$ $\begin{array}{lll}-0.05683 & 1.33999 & 1.25740\end{array}$ $\begin{array}{lll}0.5582 & 0.81823 & 1.96054\end{array}$ $\begin{array}{lll}-0.35821 & 2.54472 & 1.46079\end{array}$

$\begin{array}{lll}-5.52259 & -2.05865 & -0.13369\end{array}$ $\begin{array}{llll}-4.14567 & -2.01406 & -0.05363\end{array}$ $\begin{array}{llll}-3.46816 & -0.76594 & -0.00279\end{array}$ $\begin{array}{llll}-4.23645 & 0.44178 & -0.03702\end{array}$ $\begin{array}{llll}-5.65248 & 0.36451 & -0.12061\end{array}$ $\begin{array}{lll}-6.28359 & -0.86144 & -0.16769\end{array}$ $\begin{array}{llll}-6.03222 & -3.01695 & -0.17179\end{array}$ $\begin{array}{llll}-3.5503 & -2.92155 & -0.02748\end{array}$ $\begin{array}{llll}-3.52185 & 1.66787 & 0.01341\end{array}$ $\begin{array}{llll}-6.22866 & 1.28582 & -0.14674\end{array}$ $\begin{array}{llll}-7.36627 & -0.91491 & -0.23124\end{array}$ $\begin{array}{lll}-2.15041 & 1.6494 & 0.09257\end{array}$ $\begin{array}{lll}-1.46802 & 0.39762 & 0.12286\end{array}$ $\begin{array}{llll}-4.06775 & 2.60756 & -0.01189\end{array}$ $\begin{array}{lll}-1.57421 & 2.56561 & 0.14445\end{array}$ $\begin{array}{llll}0.03799 & 0.35364 & 0.22419\end{array}$ $\begin{array}{llll}0.47785 & 1.11157 & -0.43456\end{array}$ $\begin{array}{llll}0.38729 & -0.62907 & -0.09695\end{array}$ $\begin{array}{lll}0.57804 & 0.60954 & 1.65617\end{array}$ $\begin{array}{lll}-0.03187 & -0.33869 & 2.69601\end{array}$ $\begin{array}{lll}-1.1132 & -0.19092 & 2.74478\end{array}$ $\begin{array}{llll}0.17276 & -1.37681 & 2.42761\end{array}$ $\begin{array}{llll}0.39684 & -0.14732 & 3.68664\end{array}$ $\begin{array}{lll}0.27086 & 1.97523 & 1.96504\end{array}$ $\begin{array}{lll}0.26427 & 2.09742 & 2.92394\end{array}$ $\begin{array}{lll}2.11015 & 0.38323 & 1.60868\end{array}$ $\begin{array}{lll}2.54543 & -0.72920 & 1.34783\end{array}$ $\begin{array}{lll}3.01456 & 1.55810 & 1.88074\end{array}$ $\begin{array}{lll}2.77172 & 2.38385 & 1.20458\end{array}$ $\begin{array}{rrc}2.84770 & 1.93417 & 2.89651 \\ 4.05560 & 1.25460 & 1.76372 \\ -2.10229 & -0.76360 & 0.07616\end{array}$

$\begin{array}{lll}-4.32743 & -1.02539 & -1.90314\end{array}$

$-3.09586-1.07538-1.23535$

$\begin{array}{llll}-2.59047 & 0.07155 & -0.60769\end{array}$

$\begin{array}{llll}-3.32043 & 1.26704 & -0.65034\end{array}$

$\begin{array}{llll}-4.55138 & 1.31887 & -1.31723\end{array}$

$\begin{array}{llll}-5.05524 & 0.17164 & -1.94413\end{array}$

$-4.71204-1.90165-2.38184$

$-2.54113-1.98984-1.20475$

$\begin{array}{llll}-2.81001 & 2.40425 & -0.02284\end{array}$

$\begin{array}{llll}-5.10601 & 2.23339 & -1.34777\end{array}$

$\begin{array}{llll}-5.99522 & 0.20937 & -2.45395\end{array}$

$\begin{array}{lll}-1.58085 & 2.31674 & 0.63225\end{array}$

$\begin{array}{lll}-0.89316 & 1.09552 & 0.64367\end{array}$

$\begin{array}{lll}-1.16731 & 3.17394 & 1.12121\end{array}$

$\begin{array}{lll}0.46387 & 0.98899 & 1.3639\end{array}$

$\begin{array}{lll}0.96107 & 1.93584 & 1.32976\end{array}$

$\begin{array}{lll}0.30524 & 0.70599 & 2.38353\end{array}$

$\begin{array}{lll}1.06815 & 0.25112 & 0.87883\end{array}$

$\begin{array}{llll}-1.40947 & 0.01707 & 0.03069\end{array}$

$\begin{array}{llll}-3.5893 & 3.73219 & -0.05273\end{array}$

$\begin{array}{llll}-3.11934 & 4.72379 & -0.66871\end{array}$

$\begin{array}{llll}-4.94371 & 3.84662 & 0.6712\end{array}$

$\begin{array}{llll}-5.73126 & 3.57615 & -0.00074\end{array}$

$\begin{array}{lll}-4.95359 & 3.18921 & 1.51537\end{array}$

$\begin{array}{lll}-5.08733 & 4.85403 & 1.00198\end{array}$

$1 \mathbf{a}$

C

C

C

C

C

C

$\mathrm{H}$

$\mathrm{H}$

C

$\mathrm{H}$

$\mathrm{H}$

C

C

$\mathrm{H}$

$\mathrm{H}$

$\mathrm{N}$

C

$\mathrm{H}$

$\mathrm{H}$

$\mathrm{H}$ $\begin{array}{lll}-7.92541 & 0.00732 & 0.00026 \\ -6.55261 & 0.00732 & 0.00026 \\ -5.83067 & 1.23244 & 0.00026 \\ -6.54854 & 2.4563 & 0.00063 \\ -7.96996 & 2.42693 & 0.00079 \\ -8.64146 & 1.22896 & 0.00050 \\ -8.48614 & -0.93903 & 0.00018 \\ -5.98782 & -0.93701 & 0.00004 \\ -5.82579 & 3.68103 & 0.00075 \\ -8.5172 & 3.38139 & 0.00087 \\ -9.7411 & 1.20259 & 0.00060 \\ -4.45293 & 3.6811 & 0.00048 \\ -3.73692 & 2.45921 & 0.00002 \\ -6.39025 & 4.62557 & 0.00101 \\ -3.89224 & 4.62743 & 0.00030 \\ -4.40895 & 1.26198 & 0.00000 \\ -2.19737 & 2.49626 & -0.00024 \\ -1.85304 & 3.0072 & -0.87504 \\ -1.85284 & 3.01099 & 0.87226 \\ -1.81653 & 1.49633 & 0.00189\end{array}$ 


$\begin{array}{lrcc}13 & & & \\ \mathrm{C} & 0.31439 & 0.16838 & 0.67557 \\ \mathrm{H} & 0.25185 & -0.86608 & 0.94179 \\ \mathrm{H} & 1.28865 & 0.54012 & 0.91541 \\ \mathrm{H} & 0.13944 & 0.27768 & -0.37436 \\ \mathrm{C} & -0.49421 & 0.80759 & 2.96944 \\ \mathrm{C} & 0.88334 & 1.15934 & 3.56124 \\ \mathrm{H} & 1.64204 & 0.98443 & 2.82728 \\ \mathrm{H} & 0.89559 & 2.19006 & 3.84824 \\ \mathrm{H} & 1.06953 & 0.54792 & 4.41937 \\ \mathrm{O} & -1.41411 & 0.39163 & 3.72065 \\ \mathrm{C} & -0.74600 & 0.96490 & 1.45833 \\ \mathrm{H} & -0.68346 & 1.99937 & 1.19211 \\ \mathrm{O} & -2.04806 & 0.46809 & 1.13779 \\ \mathrm{H} & -2.53832 & 0.30817 & 1.94752\end{array}$

11 (doublet)
$\mathrm{C}$
$\mathrm{H}$
$\mathrm{H}$
$\mathrm{H}$
$\mathrm{C}$
$\mathrm{O}$

$\begin{array}{lll}-1.34078 & -0.21880 & 0.028510\end{array}$

$\begin{array}{llll}-0.64195 & -0.12428 & -0.80186\end{array}$

$\begin{array}{llll}-2.31989 & 0.18410 & -0.25701\end{array}$

$\begin{array}{lll}-1.46204 & -1.27464 & 0.29872\end{array}$

$\begin{array}{lll}-0.85933 & 0.52952 & 1.24983\end{array}$

$\begin{array}{llll}0.14135 & 1.16539 & 1.37466\end{array}$

$\begin{array}{lrrl}\text { 9 (doublet) } & & & \\ \mathrm{C} & 1.37631 & 0.75419 & 0.00002 \\ \mathrm{C} & 2.15125 & 0.72421 & -1.20372 \\ \mathrm{O} & 1.56821 & 0.74756 & -2.31451 \\ \mathrm{O} & 2.06748 & 0.72669 & 1.16489 \\ \mathrm{H} & 1.46776 & 0.74997 & 1.92499 \\ \mathrm{C} & -0.11538 & 0.81284 & 0.00002 \\ \mathrm{H} & -0.53563 & -0.05072 & 0.52755 \\ \mathrm{H} & -0.46651 & 1.70642 & 0.52808 \\ \mathrm{H} & -0.51628 & 0.82890 & -1.01769 \\ \mathrm{C} & 3.66189 & 0.66485 & -1.09702 \\ \mathrm{H} & 4.04842 & 1.53009 & -0.54709 \\ \mathrm{H} & 3.97936 & -0.22847 & -0.54777 \\ \mathrm{H} & 4.09019 & 0.64841 & -2.10042\end{array}$

\section{2 (doublet)}

\begin{tabular}{|c|c|c|}
\hline $\mathrm{C}$ & $-6.37269-0.00018$ & -0.26845 \\
\hline $\mathrm{C}$ & $\begin{array}{ll}-4.97393 & 0.06087\end{array}$ & -0.2774 \\
\hline $\mathrm{C}$ & $-4.32275 \quad 1.30127$ & -0.21889 \\
\hline $\mathrm{C}$ & $\begin{array}{ll}-5.06962 & 2.48167\end{array}$ & -0.15146 \\
\hline $\mathrm{C}$ & $-6.47086 \quad 2.41868$ & -0.14257 \\
\hline $\mathrm{C}$ & $-7.12179 \quad 1.17986$ & -0.20096 \\
\hline $\mathrm{H}$ & $-6.86804-0.94756$ & -0.31315 \\
\hline $\mathrm{H}$ & $-4.40058-0.84108$ & -0.32898 \\
\hline $\mathrm{H}$ & $\begin{array}{ll}-7.04462 & 3.32037\end{array}$ & -0.09099 \\
\hline $\mathrm{H}$ & $-8.19086 \quad 1.13583$ & -0.19397 \\
\hline $\mathrm{C}$ & $-2.98137 \quad 3.73758$ & -0.10427 \\
\hline $\mathrm{C}$ & $-2.32405 \quad 2.49665$ & -0.17409 \\
\hline $\mathrm{H}$ & $-2.38952 \quad 4.62804$ & -0.06303 \\
\hline
\end{tabular}

$\begin{array}{llll}\mathrm{C} & -0.78437 & 2.46873 & -0.18856 \\ \mathrm{H} & -0.4158 & 3.36068 & -0.65059 \\ \mathrm{H} & -0.41926 & 2.41009 & 0.81551 \\ \mathrm{H} & -0.44828 & 1.616 & -0.74066 \\ \mathrm{~N} & -2.99211 & 1.34095 & -0.22828 \\ \mathrm{C} & -4.38389 & 3.86048 & -0.08622 \\ \mathrm{H} & -4.69247 & 4.44212 & -0.92966 \\ \mathrm{C} & -4.80617 & 4.56666 & 1.21555 \\ \mathrm{O} & -5.41995 & 5.66408 & 1.16547 \\ \mathrm{C} & -4.47732 & 3.92983 & 2.5786 \\ \mathrm{H} & -4.45681 & 2.86468 & 2.47897 \\ \mathrm{H} & -3.5212 & 4.27532 & 2.91231 \\ \mathrm{H} & -5.22547 & 4.20703 & 3.29158\end{array}$

\section{8 (doublet)}

\begin{tabular}{|c|c|c|c|}
\hline C & -4.21562 & -1.3669 & 0.00025 \\
\hline $\mathrm{C}$ & -2.84282 & -1.3669 & 0.00025 \\
\hline $\mathrm{C}$ & -2.12088 & -0.14178 & 0.00025 \\
\hline $\mathrm{C}$ & -2.83875 & 1.08209 & 0.00062 \\
\hline $\mathrm{C}$ & -4.26016 & 1.05272 & 0.00077 \\
\hline $\mathrm{C}$ & -4.93167 & -0.14526 & 0.00048 \\
\hline $\mathrm{H}$ & -4.77635 & -2.31324 & 0.00017 \\
\hline $\mathrm{H}$ & -2.27803 & -2.31122 & 0.00002 \\
\hline $\mathrm{C}$ & -2.116 & 2.30681 & 0.00073 \\
\hline $\mathrm{H}$ & -4.8074 & 2.00717 & 0.00085 \\
\hline $\mathrm{H}$ & -6.03131 & -0.17163 & 0.00058 \\
\hline $\mathrm{C}$ & -0.74314 & 2.30688 & 0.00046 \\
\hline $\mathrm{C}$ & -0.02712 & 1.08499 & 0.00000 \\
\hline $\mathrm{H}$ & -2.68045 & 3.25136 & 0.00099 \\
\hline $\mathrm{H}$ & -0.18244 & 3.25321 & 0.00028 \\
\hline $\mathrm{C}$ & 1.51243 & 1.12204 & -0.00025 \\
\hline $\mathrm{H}$ & 1.85231 & 1.81857 & -0.73797 \\
\hline $\mathrm{H}$ & 1.8926 & 0.148000 & -0.22744 \\
\hline N & -0.69915 & -0.11223 & -0.00002 \\
\hline
\end{tabular}

$\begin{array}{lccc}\text { TS4 } & & & \\ \text { C } & 4.2552 & -0.92203 & 0.53679 \\ \text { C } & 3.00708 & -1.36289 & 0.15521 \\ \mathrm{C} & 1.9924 & -0.42875 & -0.20222 \\ \mathrm{C} & 2.29279 & 0.97561 & -0.16055 \\ \mathrm{C} & 3.58793 & 1.39621 & 0.23612 \\ \mathrm{C} & 4.54911 & 0.46661 & 0.57854 \\ \mathrm{H} & 5.024 & -1.63852 & 0.80966 \\ \mathrm{H} & 2.76408 & -2.42000 & 0.11807 \\ \mathrm{C} & 1.25653 & 1.87585 & -0.52481 \\ \mathrm{H} & 3.81033 & 2.45931 & 0.26762 \\ \mathrm{H} & 5.53815 & 0.7953 & 0.88274 \\ \mathrm{C} & 0.02952 & 1.38269 & -0.89026 \\ \mathrm{C} & -0.18096 & -0.0318 & -0.90979 \\ \mathrm{H} & 1.4462 & 2.94547 & -0.51145 \\ \mathrm{H} & -0.775 & 2.05026 & -1.18025 \\ \mathrm{C} & -1.46228 & -0.58583 & -1.33111 \\ \mathrm{H} & -2.10136 & 0.05819 & -1.92724 \\ \mathrm{H} & -1.43096 & -1.61884 & -1.66192 \\ \mathrm{~N} & 0.77415 & -0.90300 & -0.56983\end{array}$




$\begin{array}{lrrr}\mathrm{O} & -3.73059 & -1.37687 & -0.45872 \\ \mathrm{C} & -2.70866 & -0.95599 & 0.18025 \\ \mathrm{C} & -2.81088 & 0.49988 & 0.71551 \\ \mathrm{C} & -1.94553 & -1.92695 & 1.07089 \\ \mathrm{C} & -3.87872 & 1.38106 & 0.11787 \\ \mathrm{O} & -2.09357 & 0.85846 & 1.63436 \\ \mathrm{H} & -2.54411 & -2.10563 & 1.97174 \\ \mathrm{H} & -1.82153 & -2.87617 & 0.54515 \\ \mathrm{H} & -0.97162 & -1.54545 & 1.37577 \\ \mathrm{H} & -3.96271 & 1.24855 & -0.96276 \\ \mathrm{H} & -3.67822 & 2.42313 & 0.37167 \\ \mathrm{H} & -4.84568 & 1.08848 & 0.54413\end{array}$

TS5 $_{\text {D }}$

$\mathrm{C}$
$\mathrm{C}$
$\mathrm{C}$
$\mathrm{C}$
$\mathrm{C}$
$\mathrm{C}$

C

C

C

C

$\mathrm{H}$

$\mathrm{H}$

C

$\mathrm{H}$

$\mathrm{H}$

C

C

$\mathrm{H}$

$\mathrm{H}$

C

$\mathrm{H}$

$\mathrm{H}$

$\mathrm{N}$

$\mathrm{H}$

$\mathrm{O}$

O

C

$\mathrm{H}$

$\mathrm{H}$

$\mathrm{H}$

C

$\mathrm{H}$

$\mathrm{H}$

$\mathrm{H}$

C

C

$\mathrm{H}$

$\mathrm{H}$

C

C

C

C

$\mathrm{H}$

C $\begin{array}{llll}2.87002 & -2.87999 & -1.01657\end{array}$

$\begin{array}{llll}1.9666 & -2.4867 & -0.05233\end{array}$

$\begin{array}{llll}1.67925 & -1.10634 & 0.14292\end{array}$

$\begin{array}{llll}2.34175 & -0.13459 & -0.6804\end{array}$

$\begin{array}{llll}3.26835 & -0.57132 & -1.66154\end{array}$

$\begin{array}{llll}3.52751 & -1.91726 & -1.8271\end{array}$

$\begin{array}{llll}3.08237 & -3.93487 & -1.16228\end{array}$

$\begin{array}{llll}1.45182 & -3.20891 & 0.57341\end{array}$

$\begin{array}{llll}2.01779 & 1.23188 & -0.46394\end{array}$

$\begin{array}{llll}3.76731 & 0.16926 & -2.28096\end{array}$

$\begin{array}{llll}4.23641 & -2.2457 & -2.58104\end{array}$

$\begin{array}{llll}1.0977 & 1.56484 & 0.49852\end{array}$

$\begin{array}{llll}0.48672 & 0.52989 & 1.27443\end{array}$

$\begin{array}{llll}2.49964 & 1.99773 & -1.06586\end{array}$

$\begin{array}{llll}0.8258 & 2.59938 & 0.68176\end{array}$

$\begin{array}{lll}-0.55539 & 0.85578 & 2.25856\end{array}$

$\begin{array}{llll}-0.72558 & 0.07766 & 3.0024\end{array}$

$\begin{array}{llll}-0.5022 & 1.85967 & 2.68418\end{array}$

$\begin{array}{lll}0.7734 & -0.75899 & 1.09645\end{array}$

$\begin{array}{lll}-1.64074 & 0.92629 & 1.63134\end{array}$

$\begin{array}{llll}-2.78953 & 1.08157 & 0.99873\end{array}$

$\begin{array}{llll}-4.6124 & 0.20683 & -0.2184\end{array}$

$\begin{array}{llll}-5.59899 & -0.50432 & -0.20147\end{array}$

$\begin{array}{llll}-3.86011 & -0.87394 & 1.96031\end{array}$

$\begin{array}{lll}-4.38831 & -1.79479 & 1.71155\end{array}$

$\begin{array}{lll}-4.52341 & -0.22169 & 2.53487\end{array}$

$\begin{array}{llll}-2.99046 & -1.12509 & 2.57348\end{array}$

$\begin{array}{llll}-4.4861 & 1.40895 & -1.11563\end{array}$

$\begin{array}{llll}-4.52797 & 2.3088 & -0.49169\end{array}$

$\begin{array}{llll}-3.5132 & 1.42292 & -1.61472\end{array}$

$\begin{array}{llll}-5.29925 & 1.41955 & -1.84297\end{array}$

$\begin{array}{llll}-3.38828 & -0.14455 & 0.70058\end{array}$

$\begin{array}{llll}-2.40763 & -1.02108 & -0.16269\end{array}$

$\begin{array}{lll}-1.5598 & -1.2532 & 0.4857\end{array}$

$\begin{array}{llll}-2.03861 & -0.39337 & -0.97975\end{array}$

$\begin{array}{llll}-2.96669 & -2.31438 & -0.70133\end{array}$

$\begin{array}{llll}-3.54238 & -2.36112 & -2.00484\end{array}$

$\begin{array}{llll}-3.40098 & -4.56551 & -0.36763\end{array}$

$\begin{array}{llll}-4.05502 & -3.54454 & -2.47798\end{array}$

$\begin{array}{llll}-3.56957 & -1.46185 & -2.61183\end{array}$

$\begin{array}{llll}-3.32449 & -5.70421 & 0.47977\end{array}$

\begin{tabular}{|c|c|c|}
\hline $\mathrm{C}$ & $-4.00307-4.70289$ & -1.65988 \\
\hline $\mathrm{H}$ & $-4.49893-3.60584$ & -3.46812 \\
\hline $\mathrm{C}$ & $-3.82536-6.92013$ & 0.06248 \\
\hline $\mathrm{H}$ & $-2.86569-5.58502$ & 1.45644 \\
\hline $\mathrm{C}$ & $-4.5085 \quad-5.96799$ & -2.06267 \\
\hline $\mathrm{C}$ & $-4.42232-7.0553$ & -1.21811 \\
\hline $\mathrm{H}$ & $-3.76465-7.7845$ & 0.71726 \\
\hline $\mathrm{H}$ & $-4.96428-6.06344$ & -3.04485 \\
\hline $\mathrm{H}$ & $-4.81169-8.01978$ & -1.52995 \\
\hline 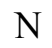 & $\begin{array}{lll}-2.89322 & -3.37948\end{array}$ & 0.08218 \\
\hline
\end{tabular}

TS8 D $_{\text {}}$

C

C

C

C

C

$\mathrm{C}$

$\mathrm{H}$

$\mathrm{H}$

$\mathrm{H}$

$\mathrm{H}$

C

C

$\mathrm{H}$

C

$\mathrm{H}$

$\mathrm{H}$

$\mathrm{H}$

$\mathrm{N}$

C

$\mathrm{H}$

C

$\mathrm{O}$

C

$\mathrm{H}$

$\mathrm{H}$

TS9

$\mathrm{C}$

C

$\mathrm{C}$
$\mathrm{C}$

$\mathrm{C}$
$\mathrm{C}$

C

$\mathrm{H}$

$\mathrm{H}$

$\mathrm{H}$

$\mathrm{H}$

C

C

$\mathrm{H}$

C

$\mathrm{H}$

$\begin{array}{lll}-6.26009 & -0.04267 & -0.42364\end{array}$

$\begin{array}{llll}-4.88578 & 0.06191 & -0.31197\end{array}$

$\begin{array}{llll}-4.25132 & 1.33215 & -0.33248\end{array}$

$\begin{array}{llll}-5.06227 & 2.50138 & -0.4563\end{array}$

$\begin{array}{llll}-6.46432 & 2.36751 & -0.57449\end{array}$

$\begin{array}{llll}-7.05823 & 1.11664 & -0.5616\end{array}$

$-6.73151-1.02116-0.41056$

$\begin{array}{lll}-4.25961 & -0.81977 & -0.21422\end{array}$

$\begin{array}{llll}-7.07276 & 3.26322 & -0.67427\end{array}$

$\begin{array}{llll}-8.13595 & 1.02376 & -0.65644\end{array}$

$\begin{array}{llll}-2.98857 & 3.77772 & -0.48575\end{array}$

$\begin{array}{llll}-2.27779 & 2.56537 & -0.35121\end{array}$

$\begin{array}{llll}-2.44468 & 4.71177 & -0.58455\end{array}$

$\begin{array}{llll}-0.76844 & 2.56736 & -0.3043\end{array}$

$\begin{array}{llll}-0.36731 & 1.73359 & -0.88698\end{array}$

$\begin{array}{lll}-0.355 & 3.5039 & -0.68804\end{array}$

$\begin{array}{llll}-0.4191 & 2.44339 & 0.72818\end{array}$

$\begin{array}{llll}-2.88054 & 1.37882 & -0.25889\end{array}$

$\begin{array}{llll}-4.39584 & 3.78188 & -0.43074\end{array}$

$\begin{array}{llll}-4.9428 & 4.65904 & -0.7641\end{array}$

$\begin{array}{llll}-4.69826 & 4.54392 & 1.60966\end{array}$

$\begin{array}{lll}-4.77085 & 5.72906 & 1.71213\end{array}$

$\begin{array}{llll}-4.54978 & 3.49373 & 2.66901\end{array}$

$\begin{array}{lll}-5.42414 & 2.83657 & 2.63579\end{array}$

$\begin{array}{lll}-3.67301 & 2.87972 & 2.44574\end{array}$

$\begin{array}{lll}-4.45696 & 3.9528 & 3.65809\end{array}$

$\begin{array}{lll}-6.53763 & 0.85228 & -1.56791\end{array}$

$\begin{array}{lll}-5.17665 & 0.66196 & -1.396\end{array}$

$\begin{array}{llll}-4.36235 & 1.68574 & -0.84935\end{array}$

$\begin{array}{llll}-4.96583 & 2.92118 & -0.44058\end{array}$

$\begin{array}{llll}-6.35332 & 3.08767 & -0.63455\end{array}$

$\begin{array}{llll}-7.1282 & 2.0694 & -1.18507\end{array}$

$\begin{array}{llll}-7.14717 & 0.06469 & -2.00018\end{array}$

$\begin{array}{llll}-4.69308 & -0.26359 & -1.69135\end{array}$

$\begin{array}{llll}-6.82514 & 4.01005 & -0.32806\end{array}$

$\begin{array}{llll}-8.19295 & 2.22587 & -1.32533\end{array}$

$\begin{array}{lll}-2.67227 & 3.6254 & 0.14347\end{array}$

$\begin{array}{lll}-2.20723 & 2.374 & -0.26583\end{array}$

$\begin{array}{lll}-1.96063 & 4.34543 & 0.52782\end{array}$

$\begin{array}{llll}-0.74421 & 2.01748 & -0.16924\end{array}$

$\begin{array}{lll}-0.15796 & 2.82369 & 0.27852\end{array}$ 


$\begin{array}{llll}\mathrm{H} & -0.61472 & 1.11237 & 0.43288 \\ \mathrm{H} & -0.34156 & 1.80638 & -1.16582 \\ \mathrm{~N} & -3.0206 & 1.42887 & -0.75449 \\ \mathrm{C} & -4.06251 & 3.99554 & 0.00811 \\ \mathrm{C} & -4.56306 & 5.12298 & 0.90574 \\ \mathrm{O} & -5.72023 & 5.16982 & 1.29666 \\ \mathrm{C} & -3.57836 & 6.2145 & 1.2685 \\ \mathrm{H} & -2.90257 & 5.85943 & 2.05508 \\ \mathrm{H} & -2.96701 & 6.5126 & 0.41138 \\ \mathrm{H} & -4.13284 & 7.07372 & 1.64749 \\ \mathrm{H} & -4.06038 & 4.72038 & -1.0997 \\ \mathrm{O} & -4.38821 & 5.38342 & -2.16335 \\ \mathrm{C} & -5.17727 & 4.76245 & -2.94864 \\ \mathrm{C} & -4.74353 & 3.57554 & -3.74685 \\ \mathrm{H} & -3.73773 & 3.27157 & -3.45421 \\ \mathrm{H} & -5.44294 & 2.74372 & -3.6144 \\ \mathrm{H} & -4.75293 & 3.82608 & -4.81512 \\ \mathrm{C} & -6.57447 & 5.2819 & -3.10714 \\ \mathrm{C} & -6.98717 & 6.45644 & -2.2549 \\ \mathrm{H} & -6.73878 & 6.29096 & -1.20294 \\ \mathrm{H} & -6.43661 & 7.35008 & -2.57118 \\ \mathrm{H} & -8.05793 & 6.62853 & -2.37047 \\ \mathrm{O} & -7.31944 & 4.76019 & -3.93681 \\ & & & \end{array}$

\section{0 (doublet)}

$\begin{array}{lccc}\mathrm{C} & -5.62026 & -2.1275 & 0.00025 \\ \mathrm{C} & -4.24746 & -2.1275 & 0.00025 \\ \mathrm{C} & -3.52552 & -0.90238 & 0.00025 \\ \mathrm{C} & -4.24339 & 0.32148 & 0.00062 \\ \mathrm{C} & -5.66481 & 0.29211 & 0.00077 \\ \mathrm{C} & -6.33631 & -0.90587 & 0.00048 \\ \mathrm{H} & -6.18099 & -3.07385 & 0.00017 \\ \mathrm{H} & -3.68267 & -3.07183 & 0.00002 \\ \mathrm{C} & -3.52064 & 1.54621 & 0.00073 \\ \mathrm{H} & -6.21205 & 1.24657 & 0.00085 \\ \mathrm{H} & -7.43595 & -0.93223 & 0.00058 \\ \mathrm{C} & -2.14778 & 1.54628 & 0.00046 \\ \mathrm{C} & -1.43177 & 0.32438 & 0.00000 \\ \mathrm{H} & -4.0851 & 2.49075 & 0.00099 \\ \mathrm{H} & -1.58709 & 2.49261 & 0.00028 \\ \mathrm{C} & 0.10779 & 0.36144 & -0.00025 \\ \mathrm{H} & 0.44767 & 1.05797 & -0.73797 \\ \mathrm{H} & 0.48796 & -0.61260 & -0.22744 \\ \mathrm{C} & 0.61099 & 0.79790 & 1.38823 \\ \mathrm{C} & -0.07307 & -0.05424 & 2.47336 \\ \mathrm{H} & -1.02208 & 0.37500 & 2.71836 \\ \mathrm{H} & -0.21555 & -1.04977 & 2.10794 \\ \mathrm{H} & 0.54313 & -0.08004 & 3.34773 \\ \mathrm{O} & 0.29707 & 2.17729 & 1.59708 \\ \mathrm{C} & 2.13634 & 0.60100 & 1.46666 \\ \mathrm{O} & 2.72839 & -0.10785 & 0.61192 \\ \mathrm{C} & 2.93991 & 1.27388 & 2.59499 \\ \mathrm{H} & 2.80509 & 2.3342 & 2.54548 \\ \mathrm{H} & 2.59495 & 0.91431 & 3.54189 \\ \mathrm{H} & 3.978 & 1.04065 & 2.48156\end{array}$

TS3 $_{\text {D }}$

$\mathrm{C}$

C

C

C

C

C

$\mathrm{H}$

$\mathrm{H}$

C

$\mathrm{H}$

$\mathrm{H}$

C

C

$\mathrm{H}$

$\mathrm{H}$

C

$\mathrm{H}$

$\mathrm{H}$

$\mathrm{N}$

$\mathrm{H}$

$\mathrm{O}$

C

C

C

$\mathrm{H}$

$\mathrm{H}$

$\mathrm{H}$

$\mathrm{C}$

$\mathrm{H}$

$\mathrm{H}$

$\mathrm{H}$

$\mathrm{O}$

$\mathrm{H}$

\section{Diacetyl ( $\left.T_{1}\right)$}

C

$\mathrm{H}$

$\mathrm{H}$

$\mathrm{H}$

$\mathrm{H}$

C

C

$\mathrm{H}$

$\mathrm{H}$

$\mathrm{H}$

$\mathrm{O}$

$\mathrm{O}$

$\begin{array}{lll}-1.35965 & -0.2193 & 0.00000\end{array}$

$\begin{array}{llll}-0.65485 & -0.09623 & -0.79562\end{array}$

$\begin{array}{llll}-2.30222 & 0.19502 & -0.29122\end{array}$

$\begin{array}{lll}-1.47855 & -1.26108 & 0.21319\end{array}$

$\begin{array}{lll}-0.84631 & 0.50666 & 1.2574\end{array}$

$\begin{array}{lll}-1.68071 & 0.48297 & 2.55155\end{array}$

$\begin{array}{lll}-1.16737 & 1.20892 & 3.80895\end{array}$

$\begin{array}{lll}-0.22572 & 0.79331 & 4.10130\end{array}$

$\begin{array}{lll}-1.04663 & 2.25038 & 3.59521\end{array}$

$\begin{array}{llll}-1.87309 & 1.08748 & 4.60401\end{array}$

$\begin{array}{lll}-2.78057 & -0.12757 & 2.5851\end{array}$

$\begin{array}{lll}0.25265 & 1.11916 & 1.23101\end{array}$ 


TS7
C
C
C
$\mathrm{H}$
$\mathrm{H}$
$\mathrm{H}$
$\mathrm{C}$
$\mathrm{H}$
$\mathrm{H}$
$\mathrm{H}$
$\mathrm{O}$
$\mathrm{O}$

TS1 $_{\text {T }}$

$\begin{array}{lccc}\mathrm{C} & -4.12403 & -1.69366 & -0.26105 \\ \mathrm{C} & -2.99548 & -1.19393 & -0.87233 \\ \mathrm{C} & -2.30836 & -0.07667 & -0.31369 \\ \mathrm{C} & -2.81181 & 0.51673 & 0.89565 \\ \mathrm{C} & -3.97478 & -0.02171 & 1.49937 \\ \mathrm{C} & -4.6186 & -1.10488 & 0.93273 \\ \mathrm{H} & -4.6431 & -2.5448 & -0.69091 \\ \mathrm{H} & -2.60271 & -1.63036 & -1.78504 \\ \mathrm{C} & -2.09475 & 1.62357 & 1.42665 \\ \mathrm{H} & -4.35131 & 0.42828 & 2.4139 \\ \mathrm{H} & -5.50915 & -1.51216 & 1.40143 \\ \mathrm{C} & -0.97635 & 2.07906 & 0.77739 \\ \mathrm{C} & -0.55472 & 1.43868 & -0.43269 \\ \mathrm{H} & -2.44215 & 2.09781 & 2.34037 \\ \mathrm{H} & -0.41178 & 2.92441 & 1.15676 \\ \mathrm{C} & 0.63773 & 1.90152 & -1.14038 \\ \mathrm{H} & 0.92036 & 2.94031 & -0.95918 \\ \mathrm{H} & 0.68103 & 1.60894 & -2.18947 \\ \mathrm{~N} & -1.19901 & 0.3839 & -0.94502 \\ \mathrm{H} & 1.63174 & 1.30333 & -0.63438 \\ \mathrm{O} & 2.72982 & 0.80456 & -0.07674 \\ \mathrm{C} & 3.08682 & -0.39053 & -0.49354 \\ \mathrm{C} & 4.24787 & -0.99033 & 0.13483 \\ \mathrm{O} & 4.64927 & -2.12443 & -0.21946 \\ \mathrm{C} & 2.33085 & -1.08704 & -1.58373 \\ \mathrm{H} & 2.43804 & -0.54695 & -2.5336 \\ \mathrm{H} & 2.72276 & -2.09683 & -1.70727 \\ \mathrm{H} & 1.26115 & -1.13125 & -1.34999 \\ \mathrm{C} & 4.95268 & -0.20768 & 1.22351 \\ \mathrm{H} & 5.29947 & 0.75897 & 0.84124 \\ \mathrm{H} & 4.26848 & 0.0011 & 2.05368 \\ \mathrm{H} & 5.80555 & -0.78127 & 1.59068\end{array}$

TS2 $_{\text {T }}$

\begin{tabular}{|c|c|}
\hline 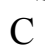 & $\begin{array}{lll}-5.52407 & -1.9114 & 0.10666\end{array}$ \\
\hline$C$ & $\begin{array}{lll}-4.13617 & -1.92331 & 0.13917\end{array}$ \\
\hline $\mathrm{C}$ & $\begin{array}{lll}-3.38626 & -0.70613 & 0.04608\end{array}$ \\
\hline 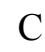 & $\begin{array}{lll}-4.11614 & 0.54082 & -0.07629\end{array}$ \\
\hline $\mathrm{C}$ & $\begin{array}{llll}-5.50776 & 0.51821 & -0.09955\end{array}$ \\
\hline & $\begin{array}{lll}-6.21769 & -0.6963 & -0.01234\end{array}$ \\
\hline
\end{tabular}

$\begin{array}{cccc}\mathrm{H} & -6.07499 & -2.84462 & 0.17355 \\ \mathrm{H} & -3.58094 & -2.85158 & 0.22957 \\ \mathrm{C} & -3.33672 & 1.75889 & -0.15717 \\ \mathrm{H} & -6.05218 & 1.45503 & -0.18650 \\ \mathrm{H} & -7.3028 & -0.68814 & -0.03738 \\ \mathrm{C} & -1.97745 & 1.68814 & -0.12277 \\ \mathrm{C} & -1.31352 & 0.41275 & -0.02365 \\ \mathrm{H} & -3.8514 & 2.71062 & -0.25208 \\ \mathrm{H} & -1.36928 & 2.58561 & -0.18762 \\ \mathrm{C} & 0.07373 & 0.31835 & 0.06059 \\ \mathrm{H} & 0.65253 & 1.18838 & -0.23206 \\ \mathrm{H} & 0.53554 & -0.65404 & -0.08553 \\ \mathrm{C} & 0.64496 & 0.42852 & 2.03150 \\ \mathrm{C} & 0.04929 & -0.81819 & 2.62789 \\ \mathrm{H} & -1.03725 & -0.82984 & 2.5199 \\ \mathrm{H} & 0.45891 & -1.70501 & 2.14403 \\ \mathrm{H} & 0.30464 & -0.85959 & 3.69453 \\ \mathrm{O} & 0.09759 & 1.6246 & 2.41134 \\ \mathrm{H} & -0.84785 & 1.51783 & 2.59641 \\ \mathrm{C} & 2.09710 & 0.49773 & 1.83367 \\ \mathrm{O} & 2.74903 & -0.55325 & 1.69136 \\ \mathrm{C} & 2.74184 & 1.86243 & 1.74304 \\ \mathrm{H} & 2.2455 & 2.48709 & 0.99128 \\ \mathrm{H} & 2.64905 & 2.39258 & 2.69741 \\ \mathrm{H} & 3.79595 & 1.74512 & 1.48770 \\ \mathrm{~N} & -2.04804 & -0.77889 & 0.08714 \\ & & & \end{array}$

\begin{tabular}{lccc}
\multicolumn{4}{l}{ diacetyl_LiClO } \\
C & 0.85939 & 0.78539 & -3.81421 \\
$\mathrm{H}$ & 1.79539 & 1.03733 & -4.31202 \\
$\mathrm{H}$ & 0.04749 & 1.42974 & -4.16940 \\
$\mathrm{H}$ & 0.55676 & -0.24042 & -4.05011 \\
$\mathrm{C}$ & 1.00235 & 0.93871 & -2.33572 \\
$\mathrm{C}$ & -0.24194 & 0.60415 & -1.46051 \\
$\mathrm{C}$ & -0.12191 & 0.79970 & 0.01873 \\
$\mathrm{H}$ & 0.71511 & 0.21331 & 0.41239 \\
$\mathrm{H}$ & 0.09916 & 1.84944 & 0.24232 \\
$\mathrm{H}$ & -1.04985 & 0.50009 & 0.50565 \\
$\mathrm{O}$ & -1.24259 & 0.20637 & -2.03083 \\
$\mathrm{O}$ & 2.04022 & 1.30756 & -1.79711 \\
$\mathrm{Li}$ & 3.16616 & 1.95005 & -0.36679 \\
$\mathrm{Cl}$ & 2.07087 & 3.26917 & 2.06265 \\
$\mathrm{O}$ & 3.11816 & 2.28337 & 1.52761 \\
$\mathrm{O}$ & 1.14834 & 2.52980 & 2.96775 \\
$\mathrm{O}$ & 1.32232 & 3.81610 & 0.87990 \\
$\mathrm{O}$ & 2.77622 & 4.35915 & 2.78975
\end{tabular}

\section{diacetyl_LiClO 4 (triplet)}

$\begin{array}{llll}\mathrm{C} & 0.89050 & 0.60099 & -3.78839 \\ \mathrm{H} & 1.05442 & 1.50989 & -4.37694 \\ \mathrm{H} & -0.07391 & 0.15951 & -4.05109\end{array}$




\begin{tabular}{|c|c|c|c|c|c|c|c|}
\hline $\mathrm{H}$ & 1.69898 & -0.10077 & -4.01581 & $\mathrm{C}$ & -6.51322 & 2.46706 & 0.02174 \\
\hline $\mathrm{C}$ & 0.92059 & 0.94378 & -2.31968 & $\mathrm{C}$ & -7.92245 & 2.41843 & 0.13103 \\
\hline $\mathrm{C}$ & -0.28729 & 0.73738 & -1.51087 & $\mathrm{C}$ & -8.59215 & 1.21036 & 0.07809 \\
\hline $\mathrm{C}$ & -0.23369 & 1.09973 & -0.03769 & $\mathrm{H}$ & -8.39928 & -0.93958 & -0.13384 \\
\hline $\mathrm{H}$ & 0.49362 & 0.44772 & 0.45569 & $\mathrm{H}$ & -5.95007 & -0.91096 & -0.33252 \\
\hline $\mathrm{H}$ & 0.10620 & 2.13524 & 0.05338 & $\mathrm{C}$ & -5.78025 & 3.68844 & 0.06847 \\
\hline $\mathrm{H}$ & -1.21292 & 0.97773 & 0.42365 & $\mathrm{H}$ & -8.46975 & 3.34834 & 0.25740 \\
\hline $\mathrm{O}$ & -1.33687 & 0.26941 & -2.01200 & $\mathrm{H}$ & -9.67368 & 1.18121 & 0.16343 \\
\hline $\mathrm{O}$ & 1.95534 & 1.40600 & -1.78116 & $\mathrm{C}$ & -4.42034 & 3.66547 & -0.04335 \\
\hline $\mathrm{Li}$ & 3.21777 & 1.98955 & -0.48259 & $\mathrm{C}$ & -3.72984 & 2.41096 & -0.20405 \\
\hline $\mathrm{Cl}$ & 2.15654 & 3.10635 & 2.11968 & $\mathrm{H}$ & -6.31467 & 4.62617 & 0.19197 \\
\hline $\mathrm{O}$ & 3.26075 & 2.29832 & 1.42680 & $\mathrm{H}$ & -3.83815 & 4.58028 & -0.01341 \\
\hline $\mathrm{O}$ & 1.21003 & 2.15133 & 2.76567 & $\mathrm{C}$ & -2.32786 & 2.39928 & -0.31722 \\
\hline $\mathrm{O}$ & 1.44849 & 3.91298 & 1.07123 & $\mathrm{H}$ & -1.77222 & 3.32906 & -0.28157 \\
\hline $\mathrm{O}$ & 2.79311 & 3.99388 & 3.13016 & $\mathrm{H}$ & -1.76842 & 1.48015 & -0.44826 \\
\hline & & & & $\mathrm{Li}$ & -3.32139 & -0.50499 & -0.39373 \\
\hline & $\mathrm{ClO}_{4}$ & & & $\mathrm{O}$ & -2.83039 & -2.46108 & -0.08501 \\
\hline $\mathrm{C}$ & -7.94651 & 0.05432 & -0.02807 & $\mathrm{Cl}$ & -1.44456 & -2.27838 & -0.69691 \\
\hline $\mathrm{C}$ & -6.56736 & 0.04899 & -0.09939 & $\mathrm{O}$ & -0.39416 & -2.55184 & 0.31545 \\
\hline $\mathrm{C}$ & -5.83829 & 1.26638 & -0.06509 & $\mathrm{O}$ & -1.41083 & -0.80907 & -1.11059 \\
\hline $\mathrm{C}$ & -6.56219 & 2.49437 & 0.03977 & $\mathrm{O}$ & -1.28746 & -3.15118 & -1.88675 \\
\hline $\mathrm{C}$ & -7.97961 & 2.47024 & 0.11062 & $\mathrm{~N}$ & -4.41097 & 1.22978 & -0.24864 \\
\hline $\mathrm{C}$ & -8.66167 & 1.27194 & 0.07868 & & & & \\
\hline $\mathrm{H}$ & -8.48885 & -0.88573 & -0.05418 & \multicolumn{4}{|c|}{ 10_LiClO 4 (doublet) } \\
\hline $\mathrm{H}$ & -6.03910 & -0.89614 & -0.18288 & $\mathrm{C}$ & 3.91702 & -1.08260 & 1.02272 \\
\hline $\mathrm{C}$ & -5.81040 & 3.69835 & 0.06886 & $\mathrm{C}$ & 2.65605 & -1.43856 & 0.59189 \\
\hline $\mathrm{H}$ & -8.51539 & 3.41194 & 0.19118 & $\mathrm{C}$ & 1.79064 & -0.46509 & 0.02246 \\
\hline $\mathrm{H}$ & -9.74547 & 1.25722 & 0.13453 & $\mathrm{C}$ & 2.25000 & 0.88684 & -0.09852 \\
\hline $\mathrm{C}$ & -4.44215 & 3.64325 & -0.00034 & $\mathrm{C}$ & 3.55455 & 1.22238 & 0.35247 \\
\hline $\mathrm{C}$ & -3.78568 & 2.38239 & -0.09717 & $\mathrm{C}$ & 4.37134 & 0.25698 & 0.90306 \\
\hline $\mathrm{H}$ & -6.32784 & 4.65076 & 0.14552 & $\mathrm{H}$ & 4.57176 & -1.83107 & 1.45902 \\
\hline $\mathrm{H}$ & -3.84263 & 4.54730 & 0.01933 & $\mathrm{H}$ & 2.29446 & -2.45852 & 0.67744 \\
\hline $\mathrm{C}$ & -2.28016 & 2.36193 & -0.14711 & $\mathrm{C}$ & 1.35896 & 1.82677 & -0.67694 \\
\hline $\mathrm{H}$ & -1.91873 & 3.03162 & -0.93466 & $\mathrm{H}$ & 3.89586 & 2.24981 & 0.25771 \\
\hline $\mathrm{H}$ & -1.87007 & 2.73082 & 0.80028 & $\mathrm{H}$ & 5.36709 & 0.51831 & 1.24805 \\
\hline $\mathrm{H}$ & -1.87082 & 1.36842 & -0.33298 & $\mathrm{C}$ & 0.11221 & 1.41474 & -1.08314 \\
\hline $\mathrm{Li}$ & -3.50094 & -0.57945 & -0.22215 & $\mathrm{C}$ & -0.26291 & 0.05058 & -0.91584 \\
\hline $\mathrm{O}$ & -2.62393 & -2.31165 & -0.22843 & $\mathrm{H}$ & 1.67104 & 2.86103 & -0.79399 \\
\hline $\mathrm{Cl}$ & -1.19610 & -2.23536 & -0.78795 & $\mathrm{H}$ & -0.58432 & 2.11568 & -1.53223 \\
\hline $\mathrm{O}$ & -0.24291 & -2.65137 & 0.27726 & $\mathrm{C}$ & -1.62960 & -0.41966 & -1.34426 \\
\hline $\mathrm{O}$ & -0.95499 & -0.80331 & -1.18562 & $\mathrm{H}$ & -2.04684 & 0.22733 & -2.12003 \\
\hline $\mathrm{O}$ & -1.09879 & -3.13308 & -1.97111 & $\mathrm{H}$ & -1.54340 & -1.42828 & -1.76136 \\
\hline \multirow[t]{2}{*}{$\mathrm{N}$} & -4.46052 & 1.23664 & -0.13027 & $\mathrm{~N}$ & 0.54920 & -0.85467 & -0.39140 \\
\hline & & & & $\mathrm{C}$ & -2.72746 & -0.58470 & -0.21978 \\
\hline \multicolumn{4}{|c|}{ 8_LiClO 4 (doublet) } & $\mathrm{C}$ & -2.81618 & 1.02109 & 0.41632 \\
\hline $\mathrm{C}$ & -7.86666 & 0.00528 & -0.09002 & $\mathrm{C}$ & -2.32536 & -1.49110 & 0.94400 \\
\hline $\mathrm{C}$ & -6.49167 & 0.01962 & -0.19920 & $\mathrm{C}$ & -3.76347 & 1.93571 & -0.28937 \\
\hline $\mathrm{C}$ & -5.77062 & 1.24717 & -0.14416 & $\mathrm{O}$ & -2.09899 & 1.26805 & 1.33428 \\
\hline
\end{tabular}




$\begin{array}{lrrr}\mathrm{H} & -3.10495 & -1.46749 & 1.70820 \\ \mathrm{H} & -2.21977 & -2.50909 & 0.55716 \\ \mathrm{H} & -1.37561 & -1.18606 & 1.38285 \\ \mathrm{H} & -3.77928 & 1.71602 & -1.35733 \\ \mathrm{H} & -3.49120 & 2.97239 & -0.08600 \\ \mathrm{H} & -4.76073 & 1.72642 & 0.11460 \\ \mathrm{O} & -3.90960 & -0.82463 & -0.74276 \\ \mathrm{Li} & -5.48503 & -1.69270 & -0.19632 \\ \mathrm{O} & -6.73006 & -1.15493 & 1.18512 \\ \mathrm{Cl} & -6.43573 & 0.11776 & 1.98439 \\ \mathrm{O} & -6.81056 & 1.29829 & 1.14397 \\ \mathrm{O} & -4.96748 & 0.15071 & 2.28132 \\ \mathrm{O} & -7.23563 & 0.07881 & 3.23602\end{array}$

\section{$\mathrm{TS1}_{\mathrm{T}_{-} \mathrm{LiClO}_{4}}$ (triplet)}

$\begin{array}{lrrr}\mathrm{C} & -3.24940 & -1.86513 & 0.26149 \\ \mathrm{C} & -2.33280 & -1.16647 & -0.49307 \\ \mathrm{C} & -2.06755 & 0.20453 & -0.21607 \\ \mathrm{C} & -2.77591 & 0.84193 & 0.85958 \\ \mathrm{C} & -3.71331 & 0.09522 & 1.61580 \\ \mathrm{C} & -3.94569 & -1.23343 & 1.32485 \\ \mathrm{H} & -3.44287 & -2.91030 & 0.04230 \\ \mathrm{H} & -1.80903 & -1.65483 & -1.30808 \\ \mathrm{C} & -2.48952 & 2.20738 & 1.11910 \\ \mathrm{H} & -4.24228 & 0.58625 & 2.42739 \\ \mathrm{H} & -4.66312 & -1.80158 & 1.90807 \\ \mathrm{C} & -1.56111 & 2.86342 & 0.35219 \\ \mathrm{C} & -0.90170 & 2.16893 & -0.70528 \\ \mathrm{H} & -3.00708 & 2.71794 & 1.92609 \\ \mathrm{H} & -1.31934 & 3.90519 & 0.53062 \\ \mathrm{C} & 0.13367 & 2.83564 & -1.48767 \\ \mathrm{H} & 0.22706 & 3.90975 & -1.33424 \\ \mathrm{H} & 0.21917 & 2.53586 & -2.53367 \\ \mathrm{H} & 1.25837 & 2.34725 & -1.08699 \\ \mathrm{O} & 2.38242 & 1.73702 & -0.92140 \\ \mathrm{C} & 2.58097 & 1.18770 & 0.25798 \\ \mathrm{C} & 3.78282 & 0.38585 & 0.40821 \\ \mathrm{O} & 4.03263 & -0.17274 & 1.50009 \\ \mathrm{C} & 1.60548 & 1.36713 & 1.37995 \\ \mathrm{H} & 0.68378 & 0.80131 & 1.19668 \\ \mathrm{H} & 2.05256 & 1.00497 & 2.30627 \\ \mathrm{H} & 1.33197 & 2.42209 & 1.48858 \\ \mathrm{C} & 4.70041 & 0.25940 & -0.78703 \\ \mathrm{H} & 5.13214 & 1.23743 & -1.03077 \\ \mathrm{H} & 5.50273 & -0.44461 & -0.55988 \\ \mathrm{H} & 4.14481 & -0.07245 & -1.67033 \\ \mathrm{~N} & -1.14614 & 0.87284 & -0.96843 \\ \mathrm{O} & 0.99925 & 0.66439 & -4.00201\end{array}$

$\begin{array}{llll}\mathrm{Cl} & 1.81144 & -0.61514 & -4.18158 \\ \mathrm{O} & 1.33530 & -1.52104 & -3.04860 \\ \mathrm{O} & 3.25995 & -0.33063 & -4.03646 \\ \mathrm{O} & 1.51100 & -1.23486 & -5.49604 \\ \mathrm{Li} & 0.08049 & -0.08262 & -2.33175\end{array}$

\section{$\mathrm{TS}_{\mathrm{D}_{-}} \mathrm{LiClO}_{4}$ (doublet)}

$\begin{array}{lrrr}\mathrm{C} & 4.24446 & -1.11365 & 0.30674 \\ \mathrm{C} & 2.94724 & -1.48377 & 0.03106 \\ \mathrm{C} & 1.96908 & -0.49445 & -0.28155 \\ \mathrm{C} & 2.35856 & 0.89102 & -0.30733 \\ \mathrm{C} & 3.70133 & 1.23696 & -0.01820 \\ \mathrm{C} & 4.62502 & 0.25497 & 0.28298 \\ \mathrm{H} & 4.98608 & -1.86929 & 0.54624 \\ \mathrm{H} & 2.63567 & -2.52318 & 0.04538 \\ \mathrm{C} & 1.35659 & 1.84807 & -0.62031 \\ \mathrm{H} & 3.99189 & 2.28360 & -0.03403 \\ \mathrm{H} & 5.65214 & 0.52746 & 0.50493 \\ \mathrm{C} & 0.07718 & 1.42618 & -0.87596 \\ \mathrm{C} & -0.21958 & 0.02805 & -0.83360 \\ \mathrm{H} & 1.61373 & 2.90282 & -0.65139 \\ \mathrm{H} & -0.70773 & 2.13506 & -1.11648 \\ \mathrm{C} & -1.56339 & -0.45339 & -1.10231 \\ \mathrm{H} & -2.22557 & 0.20703 & -1.65411 \\ \mathrm{H} & -1.63489 & -1.50066 & -1.37914 \\ \mathrm{~N} & 0.70171 & -0.89758 & -0.53733 \\ \mathrm{C} & -2.61752 & -0.67080 & 0.54634 \\ \mathrm{C} & -2.55260 & 0.82815 & 0.97848 \\ \mathrm{C} & -1.81705 & -1.63285 & 1.40356 \\ \mathrm{C} & -3.62581 & 1.74115 & 0.45775 \\ \mathrm{O} & -1.68363 & 1.17470 & 1.75549 \\ \mathrm{H} & -2.33977 & -1.74185 & 2.36049 \\ \mathrm{H} & -1.77870 & -2.60753 & 0.91300 \\ \mathrm{H} & -0.80688 & -1.27600 & 1.59693 \\ \mathrm{H} & -3.87339 & 1.52359 & -0.58347 \\ \mathrm{H} & -3.31138 & 2.77874 & 0.57754 \\ \mathrm{H} & -4.53667 & 1.56843 & 1.04357 \\ \mathrm{O} & -3.75285 & -1.06055 & 0.10126 \\ \mathrm{Li} & -5.57436 & -1.40558 & 0.02352 \\ \mathrm{O} & -7.38066 & -0.83180 & 0.41239 \\ \mathrm{Cl} & -7.58731 & 0.50792 & 1.13025 \\ \mathrm{O} & -7.55937 & 1.59970 & 0.11174 \\ \mathrm{O} & -6.46462 & 0.68189 & 2.10849 \\ \mathrm{O} & -8.89998 & 0.46270 & 1.82858\end{array}$

\section{TS5 $_{D_{-}} \mathbf{L i C l O}_{4}$}

$\begin{array}{llll}\mathrm{C} & 2.61860 & -2.88249 & -2.21917 \\ \mathrm{C} & 1.66847 & -2.74573 & -1.23004\end{array}$




\begin{tabular}{|c|c|c|c|c|c|c|c|}
\hline $\mathrm{C}$ & 1.55951 & -1.52842 & -0.50230 & $\mathrm{Li}$ & -0.86826 & -2.84559 & 0.66614 \\
\hline $\mathrm{C}$ & 2.46951 & -0.46055 & -0.80043 & $\mathrm{Cl}$ & 0.38394 & -4.45088 & 2.80788 \\
\hline $\mathrm{C}$ & 3.43402 & -0.63206 & -1.82624 & $\mathrm{O}$ & 1.32843 & -3.51639 & 3.48445 \\
\hline $\mathrm{C}$ & 3.50511 & -1.81836 & -2.52663 & $\mathrm{O}$ & 0.99591 & -5.00854 & 1.55647 \\
\hline $\mathrm{H}$ & 2.69689 & -3.81836 & -2.76358 & $\mathrm{O}$ & -0.87214 & -3.67152 & 2.39983 \\
\hline $\mathrm{H}$ & 1.01851 & -3.57655 & -0.97948 & $\mathrm{O}$ & -0.02945 & -5.54736 & 3.72660 \\
\hline $\mathrm{C}$ & 2.35637 & 0.73095 & -0.03767 & $\mathrm{~N}$ & 0.59798 & -1.40879 & 0.46235 \\
\hline $\mathrm{H}$ & 4.11641 & 0.18404 & -2.04617 & & & & \\
\hline $\mathrm{H}$ & 4.24607 & -1.94616 & -3.30942 & & & & \\
\hline $\mathrm{C}$ & 1.40588 & 0.81902 & 0.94801 & & & & \\
\hline $\mathrm{C}$ & 0.53346 & -0.28370 & 1.18696 & & & & \\
\hline $\mathrm{H}$ & 3.02960 & 1.55979 & -0.23798 & & & & \\
\hline $\mathrm{H}$ & 1.30578 & 1.71263 & 1.55427 & & & & \\
\hline $\mathrm{C}$ & -0.50931 & -0.18897 & 2.21609 & & & & \\
\hline $\mathrm{H}$ & -0.82771 & -1.14443 & 2.63423 & & & & \\
\hline $\mathrm{H}$ & -0.32895 & 0.56594 & 2.98228 & & & & \\
\hline $\mathrm{H}$ & -1.54780 & 0.28679 & 1.66641 & & & & \\
\hline $\mathrm{O}$ & -2.61610 & 0.82957 & 1.14671 & & & & \\
\hline $\mathrm{C}$ & -4.67564 & 0.66222 & -0.02624 & & & & \\
\hline $\mathrm{O}$ & -5.70686 & 0.06261 & -0.26281 & & & & \\
\hline $\mathrm{C}$ & -3.96495 & -1.14049 & 1.59971 & & & & \\
\hline $\mathrm{H}$ & -4.71680 & -1.79890 & 1.16189 & & & & \\
\hline $\mathrm{H}$ & -4.41284 & -0.59840 & 2.43655 & & & & \\
\hline $\mathrm{H}$ & -3.14926 & -1.75323 & 1.98883 & & & & \\
\hline $\mathrm{C}$ & -4.48159 & 2.12215 & -0.32571 & & & & \\
\hline $\mathrm{H}$ & -4.35769 & 2.65747 & 0.62210 & & & & \\
\hline $\mathrm{H}$ & -3.55641 & 2.27351 & -0.89034 & & & & \\
\hline $\mathrm{H}$ & -5.34118 & 2.51157 & -0.87260 & & & & \\
\hline $\mathrm{C}$ & -3.45087 & -0.12660 & 0.57000 & & & & \\
\hline $\mathrm{C}$ & -2.73212 & -0.77715 & -0.68376 & & & & \\
\hline $\mathrm{H}$ & -1.66642 & -0.79801 & -0.45311 & & & & \\
\hline $\mathrm{H}$ & -2.84174 & -0.10956 & -1.54463 & & & & \\
\hline $\mathrm{C}$ & -3.18400 & -2.16698 & -1.04617 & & & & \\
\hline $\mathrm{C}$ & -4.31914 & -2.36511 & -1.87648 & & & & \\
\hline $\mathrm{C}$ & -2.87144 & -4.46962 & -0.82364 & & & & \\
\hline $\mathrm{C}$ & -4.72553 & -3.64387 & -2.17187 & & & & \\
\hline $\mathrm{H}$ & -4.85660 & -1.50370 & -2.25449 & & & & \\
\hline $\mathrm{C}$ & -2.12543 & -5.55226 & -0.28724 & & & & \\
\hline $\mathrm{C}$ & -4.00651 & -4.74868 & -1.64865 & & & & \\
\hline $\mathrm{H}$ & -5.59212 & -3.82040 & -2.80317 & & & & \\
\hline $\mathrm{C}$ & -2.49597 & -6.85278 & -0.56167 & & & & \\
\hline $\mathrm{H}$ & -1.26897 & -5.35194 & 0.34759 & & & & \\
\hline $\mathrm{C}$ & -4.36197 & -6.09826 & -1.91207 & & & & \\
\hline $\mathrm{C}$ & -3.62093 & -7.13144 & -1.37871 & & & & \\
\hline $\mathrm{H}$ & -1.92110 & -7.67301 & -0.14297 & & & & \\
\hline $\mathrm{H}$ & -5.22621 & -6.29907 & -2.53907 & & & & \\
\hline $\mathrm{H}$ & -3.89664 & -8.16179 & -1.58051 & & & & \\
\hline $\mathrm{N}$ & -2.48558 & -3.18156 & -0.54048 & & & & \\
\hline
\end{tabular}




\section{REFERENCES}

${ }^{1}$ Andraos, J. Relationships between step and cumulative PMI and E-factors: implications on estimating material efficiency with respect to charting synthesis optimization strategies. Green Process Synth. 2019, 8, 324-336.

2 Trost, B. M. The atom economy- a search for synthetic efficiency. Science, 1991, 254, 1471-1477.

${ }^{3}$ Andraos, J. Unification of Reaction Metrics for Green Chemistry II: Evaluation of Named Organic Reactions and Application to Reaction Discovery. Org. Process Res. Dev. 2005, 9, 404-431.

${ }^{4}$ Jiang, L.; Huang, Y.; Yan, Y.; Xie, Y. Metal-free C( $\left.\mathrm{sp}^{3}\right)-\mathrm{H}$ oxidation of 2-methylquinolines with PIDA under microwave irradiation. Tetrahedron Lett., 2016, 57, 4149-4151.

5 Pandit, R. P.; Kim, S. H.; Lee Y. R. Iron-Catalyzed Annulation of 1,2-Diamines and Diazodicarbonyls for Diverse and Polyfunctionalized Quinoxalines, Pyrazines, and Benzoquinoxalines in Water. Adv. Synth. Catal., 2016, 358, 3586-3599.

${ }^{6}$ Nibbering, N. M.M. The McLafferty rearrangement: a personal recollection. J. Am. Soc. Mass Spectrom., 2004, 15, 956-958.

${ }^{7}$ Jahangiri, S.; Timerghazin, Q.; Jiang, H.; Peslherb, G.; English, A. Dramatic C-C bond activation on protonation of the persistent nitroxyl radical TEMPO. Int. J. Mass Spectrom., 2018, 429, 182-188. 